\title{
Fundamentals of the Thermohydrogravidynamic Theory of the Global Seismotectonic Activity of the Earth
}

\author{
Sergey V. Simonenko \\ V.I. Il'ichev Pacific Oceanological Institute, Far Eastern Branch of Russian Academy of Sciences, \\ 43 Baltiyskaya Street, Vladivostok 690041, Russia \\ Correspondence should be addressed to Sergey V. Simonenko; sergeysimonenko@mail.ru
}

Received 7 November 2012; Accepted 10 April 2013

Academic Editor: Umberta Tinivella

Copyright (c) 2013 Sergey V. Simonenko. This is an open access article distributed under the Creative Commons Attribution License, which permits unrestricted use, distribution, and reproduction in any medium, provided the original work is properly cited.

\begin{abstract}
The article presents the fundamentals of the cosmic geophysics (representing the deterministic thermohydrogravidynamic theory intended for earthquakes prediction) based on the author's generalized differential formulation of the first law of thermodynamics extending the classical Gibbs' formulation by taking into account (along with the classical infinitesimal change of heat $\delta Q$ and the classical infinitesimal change of the internal energy $d U_{\tau}$ ) the infinitesimal increment of the macroscopic kinetic energy $d K_{\tau}$, the infinitesimal increment of the gravitational potential energy $d \pi_{\tau}$, the generalized expression for the infinitesimal work $\delta A_{\mathrm{np}, \partial \tau}$ done by the nonpotential terrestrial stress forces (determined by the symmetric stress tensor $\mathbf{T}$ ) acting on the boundary of the continuum region $\tau$, and the infinitesimal increment $d G$ of energy due to the cosmic and terrestrial nonstationary energy gravitational influence on the continuum region $\tau$ during the infinitesimal time $d t$. Based on the established generalized differential formulation of the first law of thermodynamics, the author explains the founded cosmic energy gravitational genesis of the strong Chinese 2008 and the strong Japanese 2011 earthquakes.
\end{abstract}

\section{Introduction}

The problem of the long-term predictions of the strong earthquakes $[1,2]$ is the significant problem of the modern geophysics. The analysis of the period 1977-1985 revealed [3] the strongly nonrandom tendencies in the earthquake-induced geodetic changes (owing to the mass redistribution of material inside the Earth) related to the change of the Earth's rotation and the Earth's gravitational field. The analysis of the period 1977-1993 (characterized by 11015 major earthquakes) revealed [4] the strong earthquakes' tendency to increase the Earth's spin (rotational) energy. The analysis of the same period 1977-1993 revealed [5] "an extremely strong tendency for the earthquakes to decrease the global gravitational energy" confirming the inherent relation of the earthquakes with the transformation of the Earth's gravitational energy into the seismic wave energy and frictional heat. The previous analysis of the principal geological features of the past $400 \times$ $10^{6}$ years revealed [6] the geological evidence for a pulsating gravitation related to periodic variation of the Earth's radius during the geological evolution of the Earth. The combination of satellite and gravimetric data revealed [7] the free-air anomalies of the Earth's gravitational field.

It is well known that "the deterministic prediction of the time of origin, hypocentral (or epicentral) location, and magnitude of an impending earthquake is an open scientific problem" [8]. It was conjectured [8] that the possible earthquake prediction and warning must be carried out on a deterministic basis. However, it was pointed out [8] with some regret that the modern "study of the physical conditions that give rise to an earthquake and the processes that precede a seismic rupture of an ordinary event are at a very preliminary stage and, consequently, the techniques of prediction of time of origin, epicentre, and magnitude of an impending earthquake now available are below standard". The authors [8] argued that "a new strong theoretical scientific effort is necessary to try to understand the physics of the earthquake". It was conjectured [8] that the present level of knowledge of the geophysical processes "is unable to achieve the objective of a deterministic prediction of an ordinary seismic event, but it certainly will in a more or less distant future tackle the problem with seriousness and avoiding scientifically incorrect, wasteful, and 
inconclusive shortcuts, as sometimes has been done". Sgrigna and Conti conjectured [8] that "it will take long time (may be years, tens of years, or centuries) because this approach requires a great cultural, financial, and organizational effort on an international basis". It was conjectured [8] that a possible contribution to a deterministic earthquake prediction approach is related to observations and physical modelling of earthquake precursors to formulate, in perspective, "a unified theory able to explain the causes of its genesis, and the dynamics, rheology, and microphysics of its preparation, occurrence, postseismic relaxation, and interseismic phases". It was conjectured [8] that "the study of the physical conditions that give rise to an earthquake and of the processes that precede a seismic rupture is at a very preliminary stage and, consequently, the techniques of prediction available at the moment are below standard". However, Sgrigna and Conti believe [8] that "it should be better to pursue the deterministic prediction approach even if a reliable deterministic method of earthquake prediction will presumably be available only in the more distant future".

It was pointed out [9] that the gravity changes (derived from regional gravity monitoring data in China from 1998 to 2005) exhibited noticeable variations before the occurrence of two large earthquakes in 2008 in the areas surrounding Yutian (Xinjiang) and Wenchuan (Sichuan). A recent research [10] by Zhan and his colleagues demonstrated that significant gravity changes were observed before all nine large earthquakes that ruptured within or near mainland China from 2001 to 2008. It was pointed out [9] that the past experience and empirical data showed that "earthquakes typically occur within one to two years after a period of significant gravity changes in the region in question". It was concluded [9] that the "additional research is needed to remove the subjective nature in the determination of the timeframe of a forecasted earthquake".

The need of the thermohydrogravidynamic approach [11, $12]$ is confirmed by previous studies $[3-5,13]$ and by noticeable variations of gravitational field identified $[9,10]$ before strong earthquakes in China from 2001 to 2008. The necessity to consider the gravitational field (during the strong earthquakes) is also related to the observations of the slow gravitational [14, 15] ground waves resulting from strong earthquakes and spreading out from the focal regions $[16,17]$ of earthquakes. Lomnitz pointed out [16] that the gravitational ground waves (related to great earthquakes) "have been regularly reported for many years and remain a controversial subject in earthquake seismology". Richter presented [18] the detailed analysis of these observations and made the conclusion that "there is almost certainly a real phenomenon of progressing or standing waves seen on soft ground in the meizoseismal areas of great earthquakes". Lomnitz presented [17] the real evidence of the existence of the slow gravitational waves in sedimentary layers during strong earthquakes. The fundamental connections of the geodynamics, seismicity, and volcanism with gravitation (and the slow gravitational ground waves resulting from strong earthquakes) are presented in the works [19-22].

It was conjectured [23] that the recent destructive earthquakes occurred in Sichuan (China, 2008), Italy (2009), Haiti
(2010), Chile (2010), New Zealand (2010), and Japan (2011) "have shown that, in present state, scientific researchers have achieved little or almost nothing in the implementation of short- and medium-term earthquake prediction, which would be useful for disaster mitigation measures". It was conjectured [23] that "this regrettable situation could be ascribed to the present poor level of achievements in earthquake forecast". It was pointed out [23] that "although many methods have been claimed to be capable of predicting earthquakes (as numerous presentations on earthquake precursors regularly show at every international meeting), the problem of formulating such predictions in a quantitative, rigorous, and repeatable way is still open". It was formulated [23] that "another problem of practical implementation of earthquake forecasting could be due to the lack of common understanding and exchange of information between the scientific community and the governmental authorities that are responsible for earthquake damage mitigation in each country: they operate in two different environments, they aim at different tasks, and they generally speak two different languages". It was pointed out [23] that "the way how seismologists should formulate their forecasts and how they should transfer them to decision-makers and to the public is still a tricky issue". It was clearly formulated [23] that "the formulation of probabilistic earthquake forecasts with large uncertainties in space and time and very low probability levels is still difficult to be used by decision-making people". It was conjectured [23] that "in real circumstances the authorities deal with critical problems related to the high cost of evacuating the population from an area where the scientific methods estimate an expected rate of destructive earthquake as one in many thousand days, while they require much more deterministic statements". In the special issue [23] of the International Journal of Geophysics, Console et al. assessed the status of the art of earthquake forecasts and their applicability. They invited authors "to report methods and case studies that could concretely contribute or, at least seemed promising, to improve the present frustrating situation, regarding the practical use of earthquake forecasts" [23].

In this article, by accepting with gratitude the personal invitation from Dr. Reem Ali and Dr. Radwa Ibrahim (representing the Editorial Office of the International Journal of Geophysics) to submit an article to the special issue on "Geophysical Methods for Environmental Studies", the author presents the fundamentals of the thermohydrogravidynamic theory intended for deterministic prediction of earthquakes. The thermohydrogravidynamic theory is based on the established generalized differential formulation [11, 12, 24-26] of the first law of thermodynamics (for moving rotating deforming compressible heat-conducting stratified macroscopic continuum region $\tau$ subjected to the nonstationary Newtonian gravity):

$$
d U_{\tau}+d K_{\tau}+d \pi_{\tau}=\delta Q+\delta A_{\mathrm{np}, \partial \tau}+d G
$$

extending the classical formulation [27] by taking into account (along with the classical infinitesimal change of heat $\delta Q$ and the classical infinitesimal change of the internal energy $\left.d U_{\tau}\right)$ the infinitesimal increment of the macroscopic 
kinetic energy $d K_{\tau}$, the infinitesimal increment of the gravitational potential energy $d \boldsymbol{\pi}_{\tau}$, the generalized expression for the infinitesimal work $\delta A_{\mathrm{np}, \partial \tau}$ done by the nonpotential terrestrial stress forces (determined by the symmetric stress tensor $\mathrm{T}$ ) acting on the boundary of the continuum region $\tau$, and the infinitesimal increment $d G$ of energy due to the cosmic and terrestrial nonstationary energy gravitational influence on the continuum region $\tau$ during the infinitesimal time $d t$.

In Section 2 we begin by considering the inherent physical incompleteness of the classical expression $[28,29]$ for the macroscopic kinetic energy per unit mass $\varepsilon_{k}$ defined (in classical nonequilibrium thermodynamics) as the sum of the macroscopic translational kinetic energy per unit mass $\varepsilon_{t}=$ $(1 / 2) \mathbf{v}^{2}$ of the mass center of a continuum region and the macroscopic internal rotational kinetic energy per unit mass $\varepsilon_{r}=(1 / 2) \theta \boldsymbol{\omega}^{2}$, where $\mathbf{v}$ is the speed of the mass center of a small continuum region, $\boldsymbol{\omega}$ is an angular velocity of internal rotation $[29,30]$, and $\theta$ is an inertia moment per unit mass of a small continuum region [28]. The classical de Groot and Mazur expression has inherent physical incompleteness [24, 31] related to the questionable assumption about the rigidlike rotation of a small continuum region. The classical de Groot and Mazur expression [28] does not consider the nonequilibrium component of the macroscopic velocity field related to the velocity shear defined by the rate of strain tensor $e_{i j}$. In Section 2 the macroscopic kinetic energy per unit mass $\varepsilon_{k}$ is presented [31] as a sum of the macroscopic translational kinetic energy per unit mass $\varepsilon_{t}=(1 / 2) \mathbf{v}^{2}$ of the mass center of a continuum region, the classical macroscopic internal rotational kinetic energy per unit mass $\varepsilon_{r}$ [28], the new macroscopic internal shear kinetic energy per unit mass $\varepsilon_{s}$ [31], and the new macroscopic internal kinetic energy of shear-rotational coupling per unit mass $\varepsilon_{s, r}^{\text {coup }}$ [31] with a small correction. The presented expression for $\varepsilon_{k}$ and its particular form for homogeneous continuum regions of spherical and cubical shapes generalized [31] the classical de Groot and Mazur expression in classical nonequilibrium thermodynamics $[28,29]$ by taking into account the new macroscopic internal shear kinetic energy per unit mass $\mathcal{E}_{s}$, which expresses the kinetic energy of irreversible dissipative shear motion, and also the new macroscopic internal kinetic energy of shearrotational coupling per unit mass $\varepsilon_{s, r}^{\text {coup }}$, which expresses the kinetic energy of local coupling between irreversible dissipative shear and reversible rigid-like rotational macroscopic continuum motions.

Following the "Statistical thermohydrodynamics of irreversible strike-slip-rotational processes" [11] and the "Thermohydrogravidynamics of the Solar System" [12], in Section 2.2 we present the generalized differential formulation of the first law of thermodynamics (in the Galilean frame of reference) for nonequilibrium shear-rotational states of the deformed finite one-component individual continuum (characterized by the symmetric stress tensor $\mathbf{T}$ ) region $\tau$ moving in the nonstationary gravitational field. In Section 2.3 we present the generalized differential formulation [11, 12] of the first law of thermodynamics (in the Galilean frame of reference) for nonequilibrium shear-rotational states of the deformed finite individual region $\tau$ of the compressible viscous Newtonian one-component continuum moving in the nonstationary gravitational field. We present the generalization [11, 12] of the classical [27] expression $\delta A_{\mathrm{np}, \partial \tau}=$ $-\delta W=-p d V$ by taking into account (for Newtonian continuum) the infinitesimal works $\delta A_{c}$ and $\delta A_{s}$, respectively, of acoustic and viscous Newtonian forces acting during the infinitesimal time interval $d t$ on the boundary surface $\partial \tau$ of the individual continuum region $\tau$ bounded by the continuum boundary surface $\partial \tau$. Based on the generalized differential formulation of the first law of thermodynamics, in Section 2.4 we present the analysis $[11,12]$ of the gravitational energy mechanism of the gravitational energy supply into the continuum region $\tau$ owing to the local time increase of the potential $\psi$ of the gravitational field inside the continuum region $\tau$ subjected to the nonstationary Newtonian gravitational field.

Following the "Statistical thermohydrodynamics of irreversible strike-slip-rotational processes" [11] and the "Thermohydrogravidynamics of the Solar System" [12], in Section 3 we present the fundamentals of the cosmic energy gravitational genesis of earthquakes. Using the evolution equation of the total mechanical energy of the macroscopic continuum region $\tau$ (of the compressible viscous Newtonian continuum), we demonstrate the physical adequacy $[11,12]$ of the rotational model [2] of the earthquake focal region for the seismic zone of the Pacific Ring. We present the thermodynamic foundation [11, 12] of the classical deformational (shear) model [1] of the earthquake focal region for the quasi-uniform medium of the Earth's crust characterized by practically constant viscosity. We present the generalized thermohydrogravidynamic shear-rotational model $[11,12]$ of the earthquake focal region by taking into account the classical macroscopic rotational kinetic energy [28, 29], the macroscopic nonequilibrium kinetic energies [24,31], and the external cosmic energy gravitational influences $[12,25,26]$ on the focal region of earthquakes.

In Section 4 we present the fundamentals of the cosmic geophysics [12] applicable for the planets of the Solar System. In Section 4.1 we consider the energy gravitational influences on the Earth of the inner planets and the outer planets of the Solar System. In Section 4.1.1 we present the relation for the energy gravitational influences (on the Earth) of the inner and the outer planets in the second approximation of the elliptical orbits of the planets of the Solar System. In Section 4.1.2 we present the evaluation of the relative maximal planetary instantaneous energy gravitational influences on the Earth in the approximation of the circular orbits of the planets of the Solar System. In Section 4.1.3 we present the evaluation of the relative maximal planetary integral energy gravitational influences on the Earth in the approximation of the circular orbits of the planets of the Solar System. In Section 4.2 we consider the energy gravitational influences on the Earth of the Moon. In Section 4.2.1 we present the evaluation of the relative maximal instantaneous energy gravitational influence of the Moon on the Earth in the second approximation of the elliptical orbits of the Earth and the Moon around the combined mass center $C_{3, \mathrm{MOON}}$ of the Earth and the Moon. In Section 4.2.2. we present the evaluation of the maximal integral energy gravitational influence of 
the Moon on the Earth in the approximation of the elliptical orbits of the Earth and the Moon around the combined mass center $C_{3, \text { MOON }}$ of the Earth and the Moon. In Section 4.3 we demonstrate the reality of the cosmic energy gravitational genesis of preparation and triggering of earthquakes owing to the energy gravitational influence on the Earth of the Moon and the planets of the Solar System. In Section 4.3.1 we demonstrate the real cosmic energy gravitational genesis of preparation of earthquakes by considering the energy gravitational influence on the Earth of Venus. In Section 4.3.2 we demonstrate the real cosmic energy gravitational genesis of triggering of the preparing earthquakes. In Section 4.4 we demonstrate the cosmic energy gravitational genesis of the seismotectonic activity induced by the nonstationary cosmic energy gravitational influences on the Earth of the Sun, the Moon, Venus, Jupiter, and Mars. In Section 4.4.1 we present the evaluations of the time periodicities of the maximal (instantaneous and integral) energy gravitational influences on the Earth of the Sun-Moon system, Venus, Jupiter, and Mars. In Section 4.4.2 we present the empirical time periodicities of the seismotectonic activity for various regions of the Earth. In Section 4.4.3 we present the set of the time periodicities of the periodic global seismotectonic (and volcanic) activity and the global climate variability of the Earth induced by the different combinations of the cosmic energy gravitational influences on the Earth of the Sun and the Moon, Venus, Jupiter, and Mars. In Section 4.5 we present the evidence of the cosmic energy gravitational genesis of the strong Chinese 2008 earthquakes. In Section 4.6 we present the evidence of the cosmic energy gravitational genesis of the strongest Japanese earthquakes near the Tokyo region. In Section 5 we present the summary of main results and conclusion.

\section{The Generalized Formulation of the First Law of Thermodynamics for Moving Rotating Deforming Compressible Heat-Conducting Macroscopic Individual Continuum Region $\tau$ Subjected to the Nonstationary Newtonian Gravitational Field}

2.1. The Generalized Expression for the Macroscopic Kinetic Energy of a Small Continuum Region in Nonequilibrium Thermodynamics. De Groot and Mazur defined the macroscopic kinetic energy per unit mass $\varepsilon_{k}$ as [28] the sum of the macroscopic translational kinetic energy per unit mass $\varepsilon_{t}=$ $(1 / 2) \mathbf{v}^{2}$ of a continuum region (particle) mass center and the macroscopic internal rotational kinetic energy per unit mass $\varepsilon_{r}=(1 / 2) \theta \boldsymbol{\omega}^{2}$ :

$$
\varepsilon_{k}=\varepsilon_{t}+\varepsilon_{r}=\frac{1}{2} \mathbf{v}^{2}+\frac{1}{2} \theta \boldsymbol{\omega}^{2},
$$

where $\mathbf{v}$ is the speed of the mass center of a small continuum region, $\boldsymbol{\omega}$ is an angular velocity of internal rotation [29], and $\theta$ is an inertia moment per unit mass of a small continuum region [28]. Gyarmati's definition [29] of the macroscopic

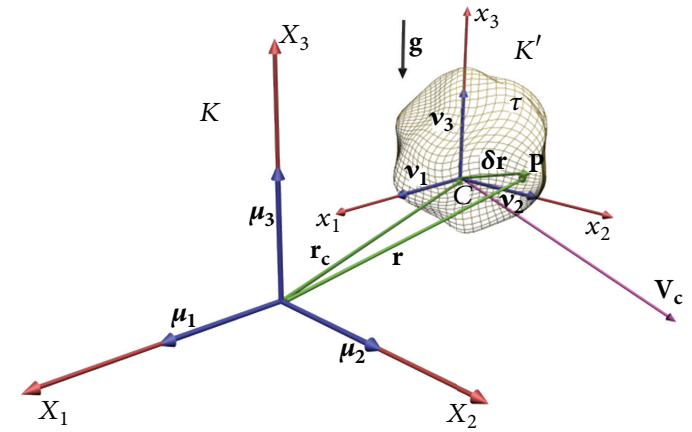

Figure 1: Cartesian coordinate system $K$ of a Galilean frame of reference and the continuum region mass center-affixed Lagrangian coordinate system $K^{\prime}$.

kinetic energy per unit mass is analogous to de Groot and Mazur's one. The classical de Groot and Mazur's and Gyarmati's definition (2) of the macroscopic kinetic energy per unit mass for a shear flows has some inherent physical incompleteness associated with the assumption about the rigid-like rotation of the continuum region with the angular velocity vector $\boldsymbol{\omega}$. This definition is based on the assumption of local thermodynamic equilibrium since it does not consider the nonequilibrium shear component of the macroscopic continuum motion related to the rate of strain tensor $e_{i j}$. However, the assumption of local thermodynamic equilibrium, as noted by de Groot and Mazur [28], may be justified only by reasonable agreement of the experimental results with the theoretical deductions based on this assumption.

Landau and Lifshitz defined [32] the macroscopic internal energy of a small macroscopic continuum region as the difference between the total kinetic energy of the continuum region and kinetic energy of the translational macroscopic motion of the continuum region. According to Landau and Lifshitz's definition [32] of the macroscopic internal energy, the term (1/2) $\theta \boldsymbol{\omega}^{2}$ in the expression (2) is the internal energy of the macroscopic (hydrodynamic) continuum motion. The classical definition [28, 29] of the macroscopic internal rotational kinetic energy per unit mass $(1 / 2) \theta \omega^{2}$ is consistent with the Landau and Lifshitz's definition of the macroscopic internal energy. We shall use further the Landau and Lifshitz's definition [32] of the macroscopic internal energy.

Following the works [11, 12, 24-26], we shall present the foundation of the generalized expression for the macroscopic kinetic energy in nonequilibrium thermodynamics. We shall assume that $\tau$ is a small individual continuum region (domain) bounded by the closed continual boundary surface $\partial \tau$ considered in the three-dimensional Euclidean space with respect to a Cartesian coordinate system $K$. We shall consider the small continuum region $\tau$ in a Galilean frame of reference with respect to a Cartesian coordinate system $K$ centred at the origin $O$ and determined by the axes $X_{1}, X_{2}$, and $X_{3}$ (see Figure 1).

The unit normal $K$-basis coordinate vectors triad $\boldsymbol{\mu}_{1}, \boldsymbol{\mu}_{2}$, and $\boldsymbol{\mu}_{3}$ is taken in the directions of the axes $X_{1}, X_{2}$, and $X_{3}$, 
respectively. The $K$-basis vector triad is taken to be righthanded in the order $\boldsymbol{\mu}_{1}, \boldsymbol{\mu}_{2}$, and $\boldsymbol{\mu}_{3}$; see Figure 1. $\mathrm{g}$ is the local gravity acceleration.

An arbitrary point $P$ in three-dimensional physical space will be uniquely defined by the position vector $\mathbf{r}=X_{i} \boldsymbol{\mu}_{i} \equiv$ $\left(X_{1}, X_{2}\right.$, and $\left.X_{3}\right)$ originating at the point $O$ and terminating at the point $P$. The continuum region-affixed Lagrangian coordinate system $K^{\prime}$ (with the axes $x_{1}, x_{2}$, and $x_{3}$ ) is centered to the mass center $C$ of the continuum region $\tau$. The axes $x_{1}, x_{2}$, and $x_{3}$ are taken parallel to the axes $X_{1}, X_{2}$, and $X_{3}$, respectively: the axis $x_{i}$ parallel to the axis $X_{i}$, where $i=1,2,3$. The unit normal $K^{\prime}$-basis coordinate vector triad $\boldsymbol{v}_{1}, \boldsymbol{v}_{2}$, and $\boldsymbol{v}_{3}$ is taken in the directions of the axes $x_{1}, x_{2}$, and $x_{3}$, respectively. The $K^{\prime}$-basis vector triad is taken to be right-handed in the order $\boldsymbol{v}_{1}, \boldsymbol{v}_{2}$, and $\boldsymbol{\nu}_{\mathbf{3}}$. The mathematical differential of the position vector $\mathbf{r}, \delta \mathbf{r} \equiv x_{i} \boldsymbol{\nu}_{i} \equiv\left(x_{1}, x_{2}\right.$, and $\left.x_{3}\right)$, expressed in terms of the coordinates $x_{i}(i=1,2,3)$ in the $K^{\prime}$-coordinate system, originates at the mass centre $C$ of the continuum region $\tau$ and terminates at the arbitrary point $P$ of the continuum region.

The position vector $\mathbf{r}_{\mathbf{c}}$ of the mass center $C$ of the continuum region $\tau$ in the $K$-coordinate system is given by the following expression:

$$
\mathbf{r}_{\mathbf{c}}=\frac{1}{m_{\tau}} \iiint_{\tau} \mathbf{r} \rho d V,
$$

where

$$
m_{\tau}=\iiint_{\tau} \rho d V
$$

is the mass of the continuum region $\tau, d V$ is the mathematical differential of physical volume of the continuum region, $\rho \equiv \rho(\mathbf{r}, t)$ is the local macroscopic density of mass distribution, $\mathbf{r}$ is the position vector of the continuum volume $d V$, and $t$ is the time. The speed of the mass centre $C$ of the continuum region $\tau$ is defined by the following expression:

$$
\mathbf{V}_{c}=\frac{d \mathbf{r}_{\mathbf{c}}}{d t}=\frac{\iiint_{\tau} \mathbf{v} \rho d V}{m_{\tau}},
$$

where $\mathbf{v}=d \mathbf{r} / d t$ is the hydrodynamic velocity vector and the operator $d / d t=(\partial / \partial t)+\mathbf{v} \cdot \nabla$ denotes the total derivative following the continuum substance [33]. The relevant threedimensional fields such as the velocity and the local mass density (and also the first and the second derivatives of the relevant fields) are assumed to vary continuously throughout the entire continuum bulk of the continuum region $\tau$. The instantaneous macroscopic kinetic energy of the continuum region $\tau$ (bounded by the continuum boundary surface $\partial \tau$ ) is the sum of the kinetic energies of small parts constituting the continuum region $\tau$ when the number of the parts, $n$, tends to infinity and the maximum from their volumes tends to zero [33]:

$$
K_{\tau} \equiv \iiint_{\tau} \frac{\rho \mathbf{v}^{2}}{2} d V,
$$

where $\mathbf{v}$ is the local hydrodynamic velocity vector, $\rho$ is the local mass density, and $d V$ is the mathematical differential of physical volume of the continuum region. We use the common Riemann's integral here and everywhere.

For the analysis of the relative continuum motion in the physical space in the vicinity of the position vector $\mathbf{r}_{\mathbf{c}}$ of the mass centre $C$ we have the Taylor series expansion (consistent with the Helmholtz's theorem [30, 34]) of the hydrodynamic velocity vector $\mathbf{v}(\mathbf{r})$ for each time moment $t$ :

$$
\begin{aligned}
\mathbf{v}\left(\mathbf{r}_{\mathbf{c}}+\delta \mathbf{r}\right)= & \mathbf{v}\left(\mathbf{r}_{\mathbf{c}}\right)+\boldsymbol{\omega}\left(\mathbf{r}_{\mathbf{c}}\right) \times \delta \mathbf{r} \\
& +\sum_{i, j=1}^{3} e_{i j}\left(\mathbf{r}_{\mathbf{c}}\right) \delta r_{j} \boldsymbol{\mu}_{i} \\
& +\frac{1}{2} \sum_{i, j, k=1}^{3} \frac{\partial^{2} v_{i}}{\partial X_{j} \partial X_{k}} \delta r_{j} \delta r_{k} \boldsymbol{\mu}_{i}+\mathbf{v}_{\text {res }},
\end{aligned}
$$

where $\mathbf{v}(\mathbf{r}) \equiv\left(v_{1}(\mathbf{r}), v_{2}(\mathbf{r}), v_{3}(\mathbf{r})\right)$ is the hydrodynamic velocity vector at the position vector $\mathbf{r}, \delta \mathbf{r} \equiv \mathbf{r}-\mathbf{r}_{\mathbf{c}} \equiv\left(\delta r_{1}, \delta r_{2}, \delta r_{3}\right) \equiv$ $\left(x_{1}, x_{2}, x_{3}\right)$ is the differential of the position vector $\mathbf{r}$,

$$
\boldsymbol{\omega}(\mathbf{r}) \equiv \frac{1}{2}(\nabla \times \mathbf{v}(\mathbf{r}))=\left(\omega_{1}, \omega_{2}, \omega_{3}\right)
$$

is the angular velocity of internal rotation (a half of the vorticity vector) in the $K$-coordinate system at the position vector r,

$$
\boldsymbol{\omega}_{v}(\mathbf{r}) \equiv(\nabla \times \mathbf{v}(\mathbf{r}))
$$

is the local vorticity in the $K$-coordinate system at the position vector $\mathbf{r}$,

$$
e_{i j}(\mathbf{r})=\frac{1}{2}\left(\frac{\partial v_{i}(\mathbf{r})}{\partial X_{j}}+\frac{\partial v_{j}(\mathbf{r})}{\partial X_{i}}\right)
$$

is the rate of strain tensor in the $K$-coordinate system at the position vector $\mathbf{r},(i, j=1,2,3)$,

$$
\nabla \equiv \mu_{1} \frac{\partial}{\partial X_{1}}+\mu_{2} \frac{\partial}{\partial X_{2}}+\mu_{3} \frac{\partial}{\partial X_{3}}
$$

is the gradient operator, and

$$
\mathbf{v}_{\mathrm{res}}=\sum_{i=1}^{3} w_{i} \boldsymbol{\mu}_{i}
$$

is the small residual part of the Taylor series expansion (7), where $w_{i}=O\left(d_{\tau}^{3}\right),(i=1,2,3), d_{\tau}=\sup _{A, B \in \partial \tau} \sqrt{(\mathbf{r}(A, B))^{2}}$ is the diameter of the continuum region $\tau$ and the vector $\mathbf{r}(A, B)$ originates at point $A$ and terminates at point $B$ of the surface $\partial \tau$. The linear of $\delta \mathbf{r}$ terms of the Taylor series expansion (7) is presented in the classical form [33]. 
Substituting formula (7) into the formula (6) and integrating by parts, then we obtain the following expression $[24,31]$ :

$$
\begin{aligned}
K_{\tau}= & K_{t}+K_{r}+K_{s}+K_{s, r}^{\text {coup }}+K_{\text {res }} \\
= & \frac{1}{2} m_{\tau} \mathbf{V}_{c}^{2}+\frac{1}{2} \sum_{i, k=1}^{3} I_{i k} \omega_{i}\left(\mathbf{r}_{\mathbf{c}}\right) \omega_{k}\left(\mathbf{r}_{\mathbf{c}}\right) \\
& +\frac{1}{2} \sum_{i, j, k=1}^{3} J_{j k} e_{i j}\left(\mathbf{r}_{\mathbf{c}}\right) e_{i k}\left(\mathbf{r}_{\mathbf{c}}\right) \\
& +\sum_{i, j, k, m=1}^{3} \varepsilon_{i j k} J_{j m} \omega_{i}\left(\mathbf{r}_{\mathbf{c}}\right) e_{k m}\left(\mathbf{r}_{\mathbf{c}}\right)+K_{\mathrm{res}},
\end{aligned}
$$

where $m_{\tau}$ is the mass of the continuum region $\tau$ and $I_{i k}$ is the $i k$-component of the classical inertia tensor depending on the mass distribution in the continuum region $\tau$ under consideration:

$$
I_{i k}=\iiint_{\tau, K^{\prime}}\left(\delta_{i k}\left(\sum_{j=1}^{3} x_{j}^{2}\right)-x_{i} x_{k}\right) \rho d V,
$$

where $x_{i}, x_{k}$ are the $i, k$-components of the vector $\delta \mathbf{r}$, respectively, in the $K^{\prime}$-coordinate system, $\delta_{i k}$ is the Kronecker delta tensor, $\varepsilon_{i j k}$ is the third-order permutation symbol, and $J_{j k}$ is the $j, k$-component classical centrifugal tensor depending on the mass distribution in the continuum region $\tau$ under consideration:

$$
J_{j k}=\iiint_{\tau, K^{\prime}} x_{j} x_{k} \rho d V
$$

$K_{\text {res }}=O\left(d_{\tau}^{7}\right)$ is a small residual part of the macroscopic kinetic energy after substituting the Taylor series expansion (7) into formula (6).

Formula (13) states that the macroscopic kinetic energy $K_{\tau}$ of the small continuum region $\tau$ is the sum of the macroscopic translational kinetic energy $K_{t}$ of the continuum region $\tau$

$$
K_{t}=\frac{1}{2} m_{\tau} \mathbf{V}_{\mathbf{c}}^{2}
$$

the macroscopic internal rotational kinetic energy $K_{r}$ of the continuum region $\tau$

$$
\begin{aligned}
K_{r} & =\frac{1}{2} \sum_{i, k=1}^{3} I_{i k} \omega_{i}\left(\mathbf{r}_{\mathbf{c}}\right) \omega_{k}\left(\mathbf{r}_{\mathbf{c}}\right) \\
& \equiv \frac{1}{2} I_{i k} \omega_{i}\left(\mathbf{r}_{\mathbf{c}}\right) \omega_{k}\left(\mathbf{r}_{\mathbf{c}}\right),
\end{aligned}
$$

the macroscopic internal shear kinetic energy $K_{s}$ of the continuum region $\tau$

$$
\begin{aligned}
K_{s} & =\frac{1}{2} \sum_{i, j, k=1}^{3} J_{j k} e_{i j}\left(\mathbf{r}_{\mathbf{c}}\right) e_{i k}\left(\mathbf{r}_{\mathbf{c}}\right) \\
& \equiv \frac{1}{2} J_{j k} e_{i j}\left(\mathbf{r}_{\mathbf{c}}\right) e_{i k}\left(\mathbf{r}_{\mathbf{c}}\right),
\end{aligned}
$$

and the macroscopic kinetic energy of shear-rotational coupling $K_{s, r}^{\text {coup }}$ of the continuum region $\tau$

$$
\begin{aligned}
K_{s, r}^{\text {coup }} & =\sum_{i, j, k, m=1}^{3} \varepsilon_{i j k} J_{j m} \omega_{i}\left(\mathbf{r}_{\mathbf{c}}\right) e_{k m}\left(\mathbf{r}_{\mathbf{c}}\right) \\
& \equiv \varepsilon_{i j k} J_{j m} \omega_{i}\left(\mathbf{r}_{\mathbf{c}}\right) e_{k m}\left(\mathbf{r}_{\mathbf{c}}\right) .
\end{aligned}
$$

The macroscopic internal rotational kinetic energy $K_{r}$ is the classical [28,29] kinetic energy of reversible (equilibrium) rigid-like macroscopic rotational continuum motion. The macroscopic internal shear kinetic energy $K_{s}$ expresses the kinetic energy of irreversible (nonequilibrium) shear continuum motion related to the rate of strain tensor $e_{i j}$. The macroscopic internal kinetic energy of the shear-rotational coupling $K_{s, r}^{\text {coup }}$ expresses the kinetic energy of the local coupling between irreversible deformation and reversible rigid-like rotation.

The deduced expression (13) for $K_{\tau}$ confirms the postulate [35] that the velocity shear $\left(e_{i j} \neq 0\right)$ represents an additional energy source taking into account the Evans, Hanley, and Hess's extended formulation [35] of the first law of thermodynamics for nonequilibrium deformed states of continuum motion. The energies $K_{r}, K_{s}, K_{s, r}^{\text {coup }}$, and $K_{\text {res }}$ are the Galilean invariants with respect to different inertial $K$-coordinate systems.

We obtained [31] from (13) the following expression for the macroscopic kinetic energy per unit mass $\varepsilon_{k}=K_{\tau} / m_{\tau}$ :

$$
\begin{aligned}
\varepsilon_{k}= & \varepsilon_{t}+\varepsilon_{r}+\varepsilon_{s}+\varepsilon_{s, r}^{\text {coup }}+\varepsilon_{\text {res }} \\
= & \frac{1}{2} \mathbf{V}_{\mathbf{c}}^{2}+\frac{1}{2} \sum_{i, k=1}^{3} \theta_{i k} \omega_{i} \omega_{k}+\frac{1}{2} \sum_{i, j, k=1}^{3} \beta_{j k} e_{i j} e_{i k} \\
& +\sum_{i, j, k, m=1}^{3} \varepsilon_{i j k} \beta_{j m} \omega_{i} e_{k m}+\varepsilon_{\text {res }},
\end{aligned}
$$

where

$$
\theta_{i k}=\frac{I_{i k}}{m_{\tau}}=\frac{I_{i k}}{\iiint_{\tau} \rho d V} \quad(i, k=1,2,3)
$$

is the $i k$-component of the classical inertia tensor per unit mass of the continuum region $\tau$,

$$
\beta_{i k}=\frac{J_{i k}}{m_{\tau}}=\frac{J_{i k}}{\iiint_{\tau} \rho d V} \quad(i, k=1,2,3)
$$

is the $i k$-component of the classical centrifugal tensor per unit mass of the continuum region $\tau$,

$$
\varepsilon_{t}=\frac{K_{t}}{m_{\tau}}=\frac{1}{2} \mathbf{V}_{\mathbf{c}}^{2}
$$

is the macroscopic translational kinetic energy per unit mass of the continuum region $\tau$ (moving as a whole at speed $\mathbf{V}_{\mathbf{c}}$ of the mass center of the continuum region $\tau$ ),

$$
\varepsilon_{r}=\frac{K_{r}}{m_{\tau}}=\frac{1}{2} \theta_{i k} \omega_{i} \omega_{k}
$$


is the macroscopic internal rotational kinetic energy per unit mass of the continuum region $\tau$,

$$
\varepsilon_{s}=\frac{K_{s}}{m_{\tau}}=\frac{1}{2} \beta_{j k} e_{i j} e_{i k}
$$

is the macroscopic internal shear kinetic energy per unit mass of the continuum region $\tau$,

$$
\varepsilon_{s, r}^{\text {coup }}=\frac{K_{s, r}^{\text {coup }}}{m_{\tau}}=\varepsilon_{i j k} \beta_{j m} \omega_{i} e_{k m}
$$

is the macroscopic internal kinetic energy of the shear-rotational coupling per unit mass (of the continuum region $\tau$ ), and $\varepsilon_{\text {res }}=O\left(d_{\tau}^{4}\right)$ is the residual correction. The energies $\varepsilon_{r}, \varepsilon_{s}$, $\varepsilon_{s, r}^{\text {coup }}$, and $\varepsilon_{\text {res }}$ are the Galilean invariants with respect to different inertial $K$-coordinate systems. We have $\varepsilon_{r}=O\left(d_{\tau}^{2}\right)$, $\varepsilon_{s}=O\left(d_{\tau}^{2}\right), \varepsilon_{s, r}^{\text {coup }}=O\left(d_{\tau}^{2}\right)$, and $\varepsilon_{\text {res }}=O\left(d_{\tau}^{4}\right)$, when $d_{\tau} \rightarrow 0$, where $d_{\tau}$ is the defined diameter of the continuum region $\tau$.

For a homogeneous continuum region of simple form (sphere or cube) we have

$$
I_{i k}=I \delta_{i k}, \quad J_{j k}=J \delta_{j k} .
$$

Formula (17) for the macroscopic internal rotational kinetic energy $K_{r}$ is reduced to the classical expression [29]

$$
K_{r}=\frac{1}{2} I \omega^{2}
$$

where $\boldsymbol{\omega}^{2}=\omega_{1}^{2}+\omega_{2}^{2}+\omega_{3}^{2}$. Formula (18) for the macroscopic internal shear kinetic energy $K_{s}$ is reduced to the expression $[24,31]$

$$
K_{s}=\frac{1}{2} J e_{i j} e_{i j} \equiv \frac{1}{2} J\left(e_{i j}\right)^{2},
$$

which is proportional to the local kinetic energy dissipation rate per unit mass $\varepsilon_{\mathrm{dis}}=2 \nu\left(e_{i j}\right)^{2}$ in an incompressible viscous Newtonian continuum, where $\nu$ is the molecular viscosity. The macroscopic internal kinetic energy of shear-rotational coupling $K_{s, r}^{\text {coup }}$ vanishes for the homogeneous continuum region $\tau$ of the form of the sphere or cube. The macroscopic kinetic energy $K_{\tau}$ for the homogeneous continuum region $\tau$ of the shape of sphere or cube is given by following expression $[24,31]$

$$
K_{\tau}=\frac{1}{2} m_{\tau} \mathbf{V}_{\mathbf{c}}^{2}+\frac{1}{2} I \omega^{2}+\frac{1}{2} J\left(e_{i j}\right)^{2}+K_{\text {res }} .
$$

Hence, the macroscopic kinetic energy per unit mass $\varepsilon_{k}$ for the homogeneous continuum sphere or cube $\tau$ is expressed as the sum of explicit terms [24, 31]

$$
\varepsilon_{k}=\frac{1}{2} \mathbf{V}_{\mathbf{c}}^{2}+\frac{1}{2} \theta \boldsymbol{\omega}^{2}+\frac{1}{2} \beta\left(e_{i j}\right)^{2}+\varepsilon_{\text {res }},
$$

where $\varepsilon_{t}=(1 / 2) \mathbf{V}_{\mathbf{c}}^{2}$ is the macroscopic translational kinetic energy per unit mass of the continuum region $\tau$; $\theta=I / m_{\tau}$; $\beta=J / m_{\tau} ; \varepsilon_{r}=(1 / 2) \theta \omega^{2}$ is the classical $[28,29]$ macroscopic internal rotational kinetic energy per unit mass of the continuum region $\tau$; $\varepsilon_{s}=(1 / 2) \beta\left(e_{i j}\right)^{2}$ is the macroscopic internal shear kinetic energy per unit mass of the homogeneous continuum sphere or cube $\tau[24,31]$.

We have the following expression for the macroscopic internal kinetic energy $K_{\text {int }}$ of the homogeneous continuum region $\tau$ of the shape of sphere or cube $[24,31]$

$$
K_{\mathrm{int}}=\frac{1}{2} I \boldsymbol{\omega}^{2}+\frac{1}{2} J\left(e_{i j}\right)^{2}+K_{\mathrm{res}}
$$

The macroscopic internal kinetic energy per unit mass $\varepsilon_{\text {int }}$ for the homogeneous continuum region $\tau$ of the shape of sphere or cube is given by the sum of explicit terms [24, 31]:

$$
\varepsilon_{\text {int }}=\frac{1}{2} \theta \boldsymbol{\omega}^{2}+\frac{1}{2} \beta\left(e_{i j}\right)^{2}+\varepsilon_{\text {res }} .
$$

Compare formula (31) with the de Groot and Mazur's definition (2). Expression (31) is reduced to de Groot and Mazur's definition (2) under condition

$$
e_{i j}=0 \quad(i, j=1,2,3)
$$

of local thermodynamic equilibrium. Therefore, we can conclude that the definition (2) of the macroscopic kinetic energy per unit mass $\varepsilon_{k}$ in classical nonequilibrium thermodynamics $[28,29]$ is based on the assumption $e_{i j}=0$ of local thermodynamic equilibrium $[24,31,35]$.

The obtained formula (20) for $\varepsilon_{k}$ and its particular form (31) (obtained for homogeneous continuum regions of spherical and cubical shapes) generalized [24, 31] the classical de Groot and Mazur expression (2) in classical nonequilibrium thermodynamics $[28,29]$ by taking into account the irreversible dissipative shear component of the macroscopic continuum motion related to the rate of strain tensor $e_{i j}$. The expression (20) for $\varepsilon_{k}$ contains the new macroscopic internal shear kinetic energy per unit mass $\varepsilon_{s}$, which expresses the kinetic energy of irreversible dissipative shear motion, and also the new macroscopic internal kinetic energy of the shearrotational coupling per unit mass $\varepsilon_{s, r}^{\text {coup }}$, which expresses the kinetic energy of local coupling between irreversible dissipative shear and reversible rigid-like rotational macroscopic continuum motions.

The macroscopic internal shear kinetic energy per unit mass (for homogeneous continuum regions of spherical and cubical shapes)

$$
\varepsilon_{s}=\frac{1}{2} \beta\left(e_{i j}\right)^{2}
$$

is proportional to the kinetic energy viscous dissipation rate per unit mass

$$
\varepsilon_{\mathrm{dis}, s}=2 \nu\left(e_{i j}\right)^{2}
$$

in an incompressible viscous Newtonian continuum characterized by the kinematic viscosity $\nu$. We have shown [24] that the proportionality

$$
\varepsilon_{s} \sim \varepsilon_{\mathrm{dis}, s}=2 \nu\left(e_{i j}\right)^{2}
$$

is the basis of the established association [36, 37] between a structure and an order (and, hence, the associated macroscopic kinetic energy), on the one hand, and irreversible dissipation, on the other hand, for the dissipative structures of turbulence in viscous Newtonian fluids. 
2.2. The Generalized Differential Formulation of the First Law of Thermodynamics (in the Galilean Frame of Reference) for Nonequilibrium Shear-Rotational States of the Deformed One-Component Individual Finite Continuum Region (Characterized by the Symmetric Stress Tensor T) Moving in the Nonstationary Newtonian Gravitational Field. Following the works $[11,12,25,26]$, we shall present the foundation of the generalized differential formulation of the first law of thermodynamics (in the Galilean frame of reference) for nonequilibrium shear-rotational states of the deformed finite one-component individual continuum region (characterized by the symmetric stress tensor $\mathbf{T}$ ) moving in the nonstationary Newtonian gravitational field. We shall consider the deformed finite one-component individual continuum region in nonequilibrium shear-rotational states characterized by the following condition:

$$
e_{i j} \neq 0 \quad(i, j=1,2,3) \text {. }
$$

Considering the graphical methods in the thermodynamics of fluids, Gibbs [27] formulated the first law of thermodynamics for the fluid body (fluid region) as follows (in Gibbs' designations):

$$
d \varepsilon=d H-d W
$$

where $d \varepsilon$ is the differential of the internal thermal energy of the fluid body, $d H$ is the differential change of heat across the boundary of the fluid body related to the thermal molecular conductivity (associated with the corresponding external or internal heat fluxes), and $d W=p d V$ is the differential work produced by the considered fluid body on its surroundings (surrounding fluid) under the differential change $d V$ of the fluid region (of volume $V$ ) characterized by the thermodynamic pressure $p$.

The formulation [32] of the first law of thermodynamics for the general thermodynamic system (material region) is given by the equivalent form (in Landau's and Lifshitz's designations [32])

$$
d E=d Q-p d V
$$

where $d A=-p d V$ is the differential work produced by the surroundings (surroundings of the thermodynamic system) on the thermodynamic system under the differential change $d V$ of volume $V$ of the thermodynamic system characterized by the thermodynamic pressure $p$; $d Q$ is the differential heat transfer (across the boundary of the thermodynamic system) related to the thermal interaction of the thermodynamic system and the surroundings (surrounding environment); $E$ is the energy of the thermodynamic system, which should contain (as supposed [32]) the kinetic energy of the macroscopic continuum motion.

We shall use the differential formulation of the first law of thermodynamics [28] for the specific volume $\vartheta=1 / \rho$ of the compressible viscous one-component deformed continuum with no chemical reactions:

$$
\frac{d u}{d t}=\frac{d q}{d t}-p \frac{d \vartheta}{d t}-\vartheta \Pi: \text { Grad } \mathbf{v}
$$

where $u$ is the specific (per unit mass) internal thermal energy, $p$ is the thermodynamic pressure, $\Pi$ is the viscous stress tensor, $\mathbf{v}$ is the hydrodynamic velocity of the continuum macrodifferential element [28], and $d q$ is the differential change of heat across the boundary of the continuum region (of unit mass) related to the thermal molecular conductivity described by the heat equation [28]:

$$
\rho \frac{d q}{d t}=-\operatorname{div} \mathbf{J}_{q}
$$

where $\mathbf{J}_{q}$ is the heat flux [28]. The viscous stress tensor $\boldsymbol{\Pi}$ is taken from the decomposition of the pressure tensor $\mathbf{P}$ [28]:

$$
\mathbf{P}=p \boldsymbol{\delta}+\Pi
$$

where $\boldsymbol{\delta}$ is the Kronecker delta tensor.

Considering the Newtonian viscous stress tensor $\mathbf{P}^{v} \equiv \Pi$ of the compressible viscous Newtonian continuum with the components [29]

$$
\Pi_{i j}=\left\{\left(\frac{2}{3} v \rho-\eta_{v}\right) \operatorname{div} \mathbf{v}\right\} \delta_{i j}-2 v \rho e_{i j}
$$

the differential formulation (41) of the first law of thermodynamics (for the continuum region (of unit mass) of the compressible viscous Newtonian one-component deformed continuum with no chemical reactions) can be rewritten as follows:

$$
\frac{d u}{d t}=\frac{d q}{d t}-p \frac{d \vartheta}{d t}+\left(v_{2}-\frac{2}{3} v\right)(\operatorname{div} \mathbf{v})^{2}+2 \nu\left(e_{i j}\right)^{2},
$$

where $v=\eta / \rho$ is the coefficient of the molecular kinematic (first) viscosity and $\nu_{2}=\eta_{v} / \rho$ is the coefficient of the molecular volume (second) viscosity [38]. The first and the second terms in the right-hand side of relation (45) are analogous to the corresponding respective first and second terms in the right-hand side of the classical formulations (39) and (40). The third term in the right-hand side of relation (45)

$$
d q_{i, c}=\left(\frac{\eta_{v}}{\rho}-\frac{2}{3} v\right)(\operatorname{div} \mathbf{v})^{2} d t
$$

is related to the "internal" heat induced during the time interval $d t$ by viscous-compressible irreversibility [24]. The fourth term in the right-hand side of relation (45)

$$
d q_{i, s}=2 \nu\left(e_{i j}\right)^{2} d t
$$

is related to the "internal" heat induced during the time interval $d t$ by viscous-shear irreversibility [24]. The differential formulation (45) of the first law of thermodynamics (for the continuum element of the compressible viscous Newtonian one-component deformed continuum with no chemical reactions) takes into account (in addition to the classical terms) the viscous-compressible irreversibility and viscous-shear irreversibility inside the continuum element of the compressible viscous Newtonian one-component deformed continuum with no chemical reactions. 
Using the differential formulation (41) of the first law of thermodynamics [28] for the total derivative $d u / d t$ (following the liquid substance) of the specific (per unit mass) internal thermal energy $u$ of a compressible viscous onecomponent deformed continuum with no chemical reactions, the heat equation (42) [28], the general equation of continuum movement [29]

$$
\frac{d \mathbf{v}}{d t}=\frac{1}{\rho} \operatorname{div} \mathbf{T}+\mathbf{g}
$$

for the deformed continuum characterized by the symmetric stress tensor $\mathbf{T}$ of general form (in particular, with the components [29]

$$
T_{i j}=-\left\{p+\left(\frac{2}{3} v \rho-\eta_{v}\right) \operatorname{div} \mathbf{v}\right\} \delta_{i j}+2 v \rho e_{i j}
$$

for the compressible viscous Newtonian one-component continuum) and taking into account the time variations of the potential $\psi$ of the nonstationary gravitational field (characterized by the local gravity acceleration vector $\mathbf{g}=-\nabla \psi$ ) inside an arbitrary finite macroscopic individual continuum region $\tau$, we derived $[11,12]$ the generalized differential formulation (for the Galilean frame of reference) of the first law of thermodynamics (for moving rotating deforming compressible heatconducting stratified macroscopic continuum region $\tau$ subjected to the nonstationary Newtonian gravitational field):

$$
\begin{aligned}
& d\left(K_{\tau}+U_{\tau}+\boldsymbol{\pi}_{\tau}\right) \\
& =d t \iint_{\partial \tau}(\mathbf{v} \cdot(\mathbf{n} \cdot \mathbf{T})) d \Omega_{\mathbf{n}} \\
& \quad-d t \iint_{\partial \tau}\left(\mathbf{J}_{q} \cdot \mathbf{n}\right) d \Omega_{\mathbf{n}}+d t \iiint_{\tau} \frac{\partial \psi}{\partial t} \rho d V,
\end{aligned}
$$

where

$$
\delta A_{\mathrm{np}, \partial \tau}=d t \iint_{\partial \tau}(\mathbf{v} \cdot(\mathbf{n} \cdot \mathbf{T})) d \Omega_{\mathbf{n}}
$$

is the differential work done during the infinitesimal time interval $d t$ by nonpotential stress forces (pressure, compressible, and viscous forces for Newtonian continuum) acting on the boundary surface $\partial \tau$ of the continuum region $\tau ; d \Omega_{\mathbf{n}}$ is the differential element (of the boundary surface $\partial \tau$ of the continuum region $\tau$ ) characterized by the external normal unit vector $\mathbf{n} ; \mathbf{t}=\mathbf{n} \cdot \mathbf{T}$ is the stress vector [29], $\mathbf{T}=-\mathbf{P}$ [29], where $\mathbf{P}$ is the pressure tensor characterized (in particular, for the model of the compressible viscous Newtonian continuum characterized by the coefficients of kinematic viscosity $v$ and the volume viscosity $\eta_{v}$ ) by components (obtained from(49))

$$
\begin{gathered}
P_{i j}=\left\{p+\left(\frac{2}{3} v \rho-\eta_{v}\right) \operatorname{div} \mathbf{v}\right\} \delta_{i j}-2 v \rho e_{i j} ; \\
\delta Q=-d t \iint_{\partial \tau}\left(\mathbf{J}_{q} \cdot \mathbf{n}\right) d \Omega_{\mathbf{n}}
\end{gathered}
$$

is the differential change of heat of the macroscopic individual continuum region $\tau$ related to the thermal molecular conductivity of heat across the boundary $\partial \tau$ of the continuum region $\tau$, where $\mathbf{J}_{q}$ is the heat flux [28] (across the element $d \Omega_{\mathbf{n}}$ of the continuum boundary surface $\partial \tau$ );

$$
\pi_{\tau} \equiv \iiint_{\tau} \psi \rho d V
$$

is the macroscopic potential energy (of the macroscopic individual continuum region $\tau$ ) related to the nonstationary potential $\psi$ of the gravitational field;

$$
U_{\tau} \equiv \iiint_{\tau} u \rho d V
$$

is the classical microscopic internal thermal energy of the macroscopic individual continuum region $\tau$;

$$
K_{\tau}=\iiint_{\tau} \frac{\rho \mathbf{v}^{2}}{2} d V
$$

is the instantaneous macroscopic kinetic energy of the macroscopic individual continuum region $\tau$. The instantaneous macroscopic kinetic energy $K_{\tau}$ is given by the relation (13) $[24,31]$ for the small macroscopic individual continuum region $\tau$.

The generalized differential formulation (50) of the first law of thermodynamics can be rewritten as follows $[11,12]$ :

$$
d U_{\tau}+d K_{\tau}+d \pi_{\tau}=\delta Q+\delta A_{\mathrm{np}, \partial \tau}+d G
$$

extending the classical [12] formulations (39) and (40)

$$
d U=\delta Q-p d V, \quad(d \varepsilon \equiv d U,-\delta W=-p d V)
$$

by taking into account (along with the classical infinitesimal change of heat $\delta Q$ and the classical infinitesimal change of the internal energy $d U_{\tau} \equiv d U$ ) the infinitesimal increment of the macroscopic kinetic energy $d K_{\tau}$, the infinitesimal increment of the gravitational potential energy $d \pi_{\tau}$, the generalized infinitesimal work $\delta A_{\mathrm{np}, \partial \tau}$ done on the continuum region $\tau$ by the surroundings of $\tau$, and the infinitesimal amount $d G$ of energy $[11,12]$

$$
d G=d t \iiint_{\tau} \frac{\partial \psi}{\partial t} \rho d V
$$

added (or lost) as the result of the Newtonian nonstationary gravitational energy influence on the continuum region $\tau$ during the infinitesimal time interval $d t$.

The generalized differential formulation (50) of the first law of thermodynamics can be rewritten as follows $[11,12]$ :

$$
\begin{aligned}
\frac{d E_{\tau}}{d t}= & \frac{d}{d t}\left(K_{\tau}+U_{\tau}+\boldsymbol{\pi}_{\tau}\right) \\
= & \iiint_{\tau}\left(\frac{1}{2} \mathbf{v}^{2}+u+\psi\right) \rho d V \\
= & \iint_{\partial \tau}(\mathbf{v} \cdot(\mathbf{n} \cdot \mathbf{T})) d \Omega_{\mathbf{n}} \\
& -\iint_{\partial \tau}\left(\mathbf{J}_{q} \cdot \mathbf{n}\right) d \Omega_{\mathbf{n}}+\iiint_{\tau} \frac{\partial \psi}{\partial t} \rho d V .
\end{aligned}
$$


The equivalent generalized differential formulations (50), (56), and (59) of the first law of thermodynamics take into account the following factors:

(1) the classical heat thermal molecular conductivity (across the boundary $\partial \tau$ of the macroscopic continuum region $\tau$ ) related to the classical infinitesimal change of heat $\delta Q$ :

$$
\delta Q=-d t \iint_{\partial \tau}\left(\mathbf{J}_{q} \cdot \mathbf{n}\right) d \Omega_{\mathbf{n}},
$$

(2) the classical infinitesimal change of the internal energy $d U_{\tau}$ of the macroscopic continuum region $\tau$ :

$$
d U_{\tau} \equiv d \iiint_{\tau} u \rho d V
$$

(3) the established [11, 12] infinitesimal increment of the macroscopic kinetic energy $d K_{\tau}$ of the macroscopic continuum region $\tau$ :

$$
d K_{\tau}=d \iiint_{\tau} \frac{\rho \mathbf{v}^{2}}{2} d V,
$$

(4) the established [11, 12] infinitesimal increment of the gravitational potential energy $d \pi_{\tau}$ of the macroscopic continuum region $\tau$ :

$$
d \pi_{\tau}=d \iiint_{\tau} \psi \rho d V
$$

(5) the established [11, 12] generalized infinitesimal work $\delta A_{\mathrm{np}, \partial \tau}$ done on the macroscopic continuum region $\tau$ by the surroundings of $\tau$ :

$$
\delta A_{\mathrm{np}, \partial \tau}=d t \iint_{\partial \tau}(\mathbf{v} \cdot(\mathbf{n} \cdot \mathbf{T})) d \Omega_{\mathbf{n}}
$$

(6) the established $[11,12]$ infinitesimal amount $d G$ of energy added (or lost) as the result of the Newtonian nonstationary gravitational energy influence on the macroscopic continuum region $\tau$ during the infinitesimal time interval $d t$ :

$$
d G=d t \iiint_{\tau} \frac{\partial \psi}{\partial t} \rho d V
$$

The generalized differential formulations (50), (56), and (59) of the first law of thermodynamics (given for the Galilean frame of reference) are valid for nonequilibrium shearrotational states of the deformed finite individual continuum region (characterized by the symmetric stress tensor $\mathbf{T}$ in the general equation (48) of continuum movement [29]) moving in the nonstationary gravitational field. The generalized differential formulations (50) and (56) of the first law of thermodynamics $[11,12]$ are the generalizations of the classical formulations (39) and (40) of the first law of thermodynamics taking into account: (1) the generalized expression (51) for the differential work $\delta A_{\mathrm{np}, \partial \tau}$ done during the infinitesimal time interval $d t$ by nonpotential stress forces acting on the boundary surface $\partial \tau$ of the individual continuum region $\tau$ and (2) the time variations of the potential $\psi$ of the nonstationary gravitational field inside the individual continuum region $\tau$ due to the deformation of the individual continuum region $\tau$ and due to the external (terrestrial and cosmic) gravitational influence on the individual continuum region $\tau$ moving in the total (internal + external) nonstationary gravitational field.

\subsection{The Generalized Differential Formulation of the First Law} of Thermodynamics (in the Galilean Frame of Reference) for Nonequilibrium Shear-Rotational States of the Deformed Finite Individual Region of the Compressible Viscous Newtonian One-Component Continuum Moving in the Nonstationary Gravitational Field. There is evidence [39] that the rocks of the Earth's crust at protracted loadings may be considered as fluids characterized by the very high viscosity. According to the classical viewpoint [39], the local mechanism of creation of the earthquakes is related to the release of the accumulated potential energy of the elastic deformation during the sudden local break (i.e., the discontinuous shear) of the Earth's crust (or the sudden increase of fluidity in the local region of the Earth's crust) accompanied by viscous relaxation and generation of seismic waves. It was conjectured [40] that "more punctual and refined methods of the mathematical analysis are obligatory" for "the practical assessment of the seismic hazard".

Taking into account the established [31] conception of the macroscopic internal shear kinetic energy (per unit mass) $\varepsilon_{s}$ related to the rate of medium deformation (i.e., with the rate of strain tensor $e_{i j}=d \varepsilon_{i j} / d t$, where $\varepsilon_{i j}$ is the deformation tensor [30]), we have elucidated [41] from the viewpoint of nonequilibrium thermodynamics the mechanism of generation of seismic waves from the separate deformed finite zone of the Earth's crust. The proportionality (37) takes place also for deformed compressible finite region of the Earth's crust for sudden rise of fluidity (in a local region of the Earth's crust) related to the local sudden medium deformation in the separate seismic zones of the seismic activity. Taking into account the established [31] proportionality (37), we have assumed [31] that the accumulated potential energy of the elastic deformation (related to the deformation tensor $\varepsilon_{i j}$ ) converts to the macroscopic internal shear kinetic energy $K_{s}$ (related to the rate of strain tensor $e_{i j}$ ) in the seismic zone simultaneously with the damping of $K_{s}$ by viscous dissipation and radiation of seismic waves during several oscillations. In Section 3 we shall evaluate this mechanism on the basis of the generalized differential formulation (50) of the first law of thermodynamics for nonequilibrium shear-rotational states of the deformed finite individual continuum region (characterized by the symmetric stress tensor T) moving in the nonstationary gravitational field.

Following the works [11, 12, 25, 26], we shall present the foundation of the generalized differential formulation of the first law of thermodynamics for nonequilibrium shearrotational states of the deformed finite individual region of the compressible viscous Newtonian one-component continuum moving in the nonstationary gravitational field. The generalized differential formulation (50) of the first law of thermodynamics (formulated for the Galilean frame of 
reference) is valid for arbitrary symmetric stress tensor $\mathbf{T}$, in particular for nonequilibrium shear-rotational states of the deformed finite individual region of the compressible viscous Newtonian one-component continuum moving in the nonstationary gravitational field. The coefficient of molecular kinematic (first, shear) viscosity $\nu=\eta / \rho$ and the coefficient of molecular volume (second) viscosity $\nu_{2}=\eta_{v} / \rho$ are assumed to vary for each time moment $t$ as an arbitrary continuous functions of the Cartesian space (three-dimensional) coordinates.

The differential work $\delta A_{\mathrm{np}, \partial \tau}$ for the Newtonian symmetric stress tensor $\mathbf{T}$ (characterized by the components (49)) is presented by three explicit terms $[11,12]$ :

$$
\begin{aligned}
\delta A_{\mathrm{np}, \partial \tau}= & \delta A_{p}+\delta A_{c}+\delta A_{s} \\
= & -d t \iint_{\partial \tau} p(\mathbf{v} \cdot \mathbf{n}) d \Omega_{\mathbf{n}} \\
& -d t \iint_{\partial \tau}\left(\frac{2}{3} \eta-\eta_{v}\right) \operatorname{div} \mathbf{v}(\mathbf{v} \cdot \mathbf{n}) d \Omega_{\mathbf{n}} \\
& +d t \iint_{\partial \tau} 2 \eta v_{\beta} n_{\alpha} e_{\alpha \beta} d \Omega_{\mathbf{n}},
\end{aligned}
$$

where

$$
\delta A_{p}=-d t \iint_{\partial \tau} p(\mathbf{v} \cdot \mathbf{n}) d \Omega_{\mathbf{n}}
$$

is the differential work of the hydrodynamic pressure forces acting on the boundary surface $\partial \tau$ of the individual continuum region $\tau$ (bounded by the continuum boundary surface $\partial \tau$ ) during the infinitesimal time interval $d t$;

$$
\delta A_{c}=-d t \iint_{\partial \tau}\left(\frac{2}{3} \eta-\eta_{v}\right) \operatorname{div} \mathbf{v}(\mathbf{v} \cdot \mathbf{n}) d \Omega_{\mathbf{n}}
$$

is the differential work (related to the combined effects of the acoustic compressibility, molecular kinematic viscosity, and molecular volume viscosity) of the acoustic (compressible) pressure forces acting on the boundary surface $\partial \tau$ of the individual continuum region $\tau$ during the infinitesimal time interval $d t$;

$$
\delta A_{s}=d t \iint_{\partial \tau} 2 \eta v_{\beta} n_{\alpha} e_{\alpha \beta} d \Omega_{\mathbf{n}}
$$

is the differential work of the viscous Newtonian forces (related to the combined effect of the velocity shear, that is, the deformation of the continuum region $\tau$, and the molecular kinematic viscosity) acting on the boundary surface $\partial \tau$ of the individual continuum region $\tau$ during the infinitesimal time interval $d t$.

Along with (45) the differential formulation of the first law of thermodynamics [28] for the total derivative $d u / d t$ (following the continuum substance) of the internal thermal energy per unit mass $u$ of the one-component deformed continuum with no chemical reactions, the thermohydrodynamic theory [28] contains additionally the equations of the mass and momentum balances:

$$
\begin{gathered}
\frac{\partial \rho}{\partial t}=-\operatorname{div} \rho \mathbf{v} \\
\rho \frac{d \mathbf{v}}{d t}=-\operatorname{Grad} p+\eta \Delta \mathbf{v}+\left(\frac{1}{3} \eta+\eta_{\nu}\right) \operatorname{Grad} \operatorname{div} \mathbf{v}
\end{gathered}
$$

The generalized differential formulation (50) of the first law of thermodynamics (together with the generalized differential work $\delta A_{\mathrm{np}, \partial \tau}$ given by the expression (66)) is valid for nonequilibrium shear-rotational states of the deformed finite individual region of the compressible viscous Newtonian one-component continuum moving in the nonstationary gravity field. The coefficient of molecular kinematic (first, shear) viscosity $\nu=\eta / \rho$ and the coefficient of molecular volume (second) viscosity $\nu_{2}=\eta_{v} / \rho$ are assumed to vary for each time moment $t$ as an arbitrary continuous functions of Cartesian space (three-dimensional) coordinates.

The generalized differential formulation (50) takes into account the dependences of the hydrodynamic pressure on the hydrodynamic vorticity $\omega_{v}$ and on the rate of strain tensor $e_{i j}$ by means of the component $\delta A_{p}$ (in the expression (66) for $\delta A_{\mathrm{np}, \partial \tau}$ ) given by the expression (67). The presence of the third term $\delta A_{s}$ (given by the expression (69) and related to the combined effect of the molecular kinematic viscosity and the deformation of the continuum region $\tau$ defined by the rate of strain tensor $e_{\alpha \beta}$ ) in the expression (66) for $\delta A_{\mathrm{np}, \partial \tau}$ generalizes essentially the classical formulations (39) and (40) of the first law of thermodynamics by taking into account the differential work of the viscous Newtonian forces acting on the boundary continuum surface $\partial \tau$ of the individual continuum region $\tau$.

The general equation (48) of continuum movement [29] for the compressible viscous Newtonian one-component continuum is reduced to the following equation:

$$
\begin{aligned}
\rho \frac{d \mathbf{v}}{d t}= & -\operatorname{Grad} p+\eta \Delta \mathbf{v} \\
& +\left(\frac{1}{3} \eta+\eta_{\nu}\right) \operatorname{Grad} \operatorname{div} \mathbf{v} \\
& +(\operatorname{Grad} \eta) \cdot \mathbf{e} \\
& -\operatorname{div} \mathbf{v} \operatorname{Grad}\left(\frac{2}{3} \eta-\eta_{\nu}\right)+\mathbf{g}
\end{aligned}
$$

where $(\operatorname{Grad} \eta) \cdot \mathbf{e}$ is the internal multiplication of the vector $(\operatorname{Grad} \eta)$ and the rate of strain tensor $\mathbf{e}$ in accordance with the corresponding definition [29]. Equation (72) generalizes the Navier-Stokes equation (71) (given for $\mathbf{g}=0$ ) by taking into account the dependences of the coefficient of molecular kinematic viscosity $\nu=\eta / \rho$ and the coefficient of molecular volume viscosity $\nu_{2}=\eta_{v} / \rho$ on the space (three-dimensional) Cartesian coordinates.

The relevant example for illustration of the significance of the term $\delta A_{s}$ (in the expression (66) for the differential work $\delta A_{\mathrm{np}, \partial \tau}$ ) is related to the thermodynamic consideration [12] of the processes of the energy exchange [42] between the oceans and the lithosphere of the Earth. According to the expression (69) for the term $\delta A_{s}$, the energy exchange between the oceans (and the atmosphere) and the lithosphere of the Earth is possible only under the presence of the medium acoustic compressibility (i.e., $\operatorname{div} \mathbf{v} \neq 0$ ) and the medium deformations (i.e., $e_{\alpha \beta} \neq 0$ ) in the boundary regions of fluid (in the oceans), air (in the atmosphere), and the compressible deformed lithosphere of the Earth. According to the generalized expression (66) for the differential work $\delta A_{\mathrm{np}, \partial \tau}$, 
the energy exchange between the oceans (and the atmosphere) and the lithosphere of the Earth is impossible for nondeformed $\left(e_{\alpha \beta}=0\right)$ and noncompressible ( $\left.\operatorname{div} \mathbf{v}=0\right)$ lithosphere.

We have the evolution equation for the total mechanical energy $\left(K_{\tau}+\pi_{\tau}\right)$ of the deformed finite individual macroscopic continuum region $\tau[11,12]$ :

$$
\begin{aligned}
\frac{d}{d t}\left(K_{\tau}+\pi_{\tau}\right)= & \frac{d}{d t} \iiint_{\tau}\left(\frac{1}{2} \mathbf{v}^{2}+\psi\right) \rho d V \\
= & \iiint_{\tau} p \operatorname{div} \mathbf{v} d V \\
& +\iiint_{\tau}\left(\frac{2}{3} \eta-\eta_{v}\right)(\operatorname{div} \mathbf{v})^{2} d V \\
& -\iiint_{\tau} 2 \nu\left(e_{i j}\right)^{2} \rho d V \\
& +\iint_{\partial \tau}(\mathbf{v} \cdot(\mathbf{n} \cdot \mathbf{T})) d \Omega_{\mathbf{n}} \\
& +\iiint_{\tau} \frac{\partial \psi}{\partial t} \rho d V
\end{aligned}
$$

obtained from the generalized differential formulation (50) for the compressible viscous Newtonian one-component continuum moving in the nonstationary gravitational field. In Section 3 we shall use the evolution equation (73) of the total mechanical energy to found the rotational, shear, and the shear-rotational models of the earthquake focal region.

2.4. Cosmic and Terrestrial Energy Gravitational Genesis of the Seismotectonic (and Volcanic) Activity of the Earth Induced by the Combined Cosmic and Terrestrial Nonstationary Energy Gravitational Influences on an Arbitrary Individual Continuum Region $\tau$ (of the Earth) and by the Nonpotential Terrestrial Stress Forces Acting on the Boundary Surface $\partial \tau$ of the Individual Continuum Region $\tau$. Following the works $[11,12]$, we shall present the physical mechanisms of the energy fluxes to the continuum region $\tau$ related to preparation of earthquakes. The equivalent generalized differential formulations (50) and (59) of the first law of thermodynamics show that the nonstationary gravitational field (related to the nonstationary gravitational potential $\psi$ ) gives the following gravitational energy power:

$$
W_{\mathrm{gr}}(\tau)=\iiint_{\tau} \frac{\partial \psi}{\partial t} \rho d V=\frac{d G}{d t}
$$

associated with the gravitational energy power of the total combined (external cosmic and terrestrial and internal related to the macroscopic continuum region $\tau$ ) gravitational field. If the macroscopic continuum region $\tau$ is not very large, consequently, it cannot induce the significant time variations to the potential $\psi$ of the gravity field inside the continuum region $\tau$. According to the equivalent generalized differential formulations (50) and (59) of the first law of thermodynamics and to the evolution equation (73) for the total mechanical energy $\left(K_{\tau}+\pi_{\tau}\right)$ of the deformed finite individual macroscopic continuum region $\tau$, the energy power of the gravitational field may produce the fractures in the continuum region $\tau$. We shall consider this aspect in Section 3.

The generalized differential formulation (59) of the first law of thermodynamics and the expression (74) for the gravitational energy power $W_{\mathrm{gr}}(\tau)$ show that the local time increase of the potential $\psi$ of the gravitational field is the gravitational energy mechanism of the gravitational energy supply into the continuum region $\tau$. Really, the local time increase of the potential $\psi$ of the gravitational field inside the continuum region $\tau(\partial \psi / \partial t>0)$ supplies the gravitational energy into the continuum region $\tau$. Consequently, according to the generalized differential formulation (59) and to the evolution equation (73), the total energy $\left(K_{\tau}+U_{\tau}+\pi_{\tau}\right)$ of the continuum region $\tau$ and the total mechanical energy $\left(K_{\tau}+\pi_{\tau}\right)$ of the continuum region $\tau$ are increased if $\partial \psi / \partial t>0$.

According to the generalized differential formulation (59) of the first law of thermodynamics and to the evolution equation (73), the gravitational energy supply into the continuum region $\tau$ may induce the formation of fractures in the continuum region $\tau$ related to the production of earthquake. This conclusion corresponds to the observations $[1,3-5,9,10$, 13] of the identified anomalous variations of the gravitational field before strong earthquakes.

According to the generalized differential formulation (59) of the first law of thermodynamics and to the evolution equation (73), the supply of energy into the continuum region $\tau$ may occur also by means of the work

$$
A_{\mathrm{np}, \partial \tau}=\int_{t_{0}}^{t} d t \iint_{\partial \tau}(\mathbf{v} \cdot(\mathbf{n} \cdot \mathbf{T})) d \Omega_{\mathbf{n}}
$$

done by nonpotential stress forces (pressure, compressible, and viscous forces for Newtonian continuum) acting on the boundary surface $\partial \tau$ of the continuum region $\tau$ during the time interval $\left(t-t_{0}\right)$.

The considered mechanisms of the energy supply to the Earth's macroscopic continuum region $\tau$ should result in the irreversible process of the splits formation in the rocks related to the generation of the high-frequency acoustic waves from the focal continuum region $\tau$ before the earthquake. Taking this into account, the sum $\delta A_{c}+\delta A_{s}$ in the expression (45) is interpreted $[11,12]$ as the energy flux (related to the compressible and viscous forces acting on the boundary surface $\partial \tau$ of the continuum region $\tau$ ) [38]

$$
\delta F_{\mathrm{vis}, c}=\delta A_{c}+\delta A_{s}
$$

directed across the boundary $\partial \tau$ (see Figures 2 and 3 ) of the continuum region $\tau$.

The considered mechanisms of the energy supply to the Earth's macroscopic continuum region $\tau$ should result in the significant increase of the energy flux $\delta F_{\text {vis }, c}$ of the geo-acoustic energy from the focal region $\tau$ before the earthquake. The deduced conclusion is in a good agreement with the results of the detailed experimental studies [43]. 


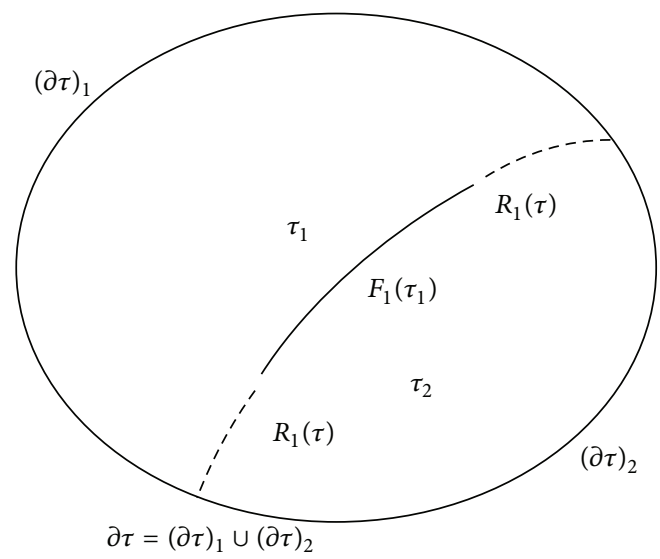

FIgURE 2: The macroscopic continuum region $\tau$ containing two subsystems $\tau_{1}$ and $\tau_{2}$ interacting on the surface $F_{1}(\tau)$ of the tangential jump of the continuum velocity.

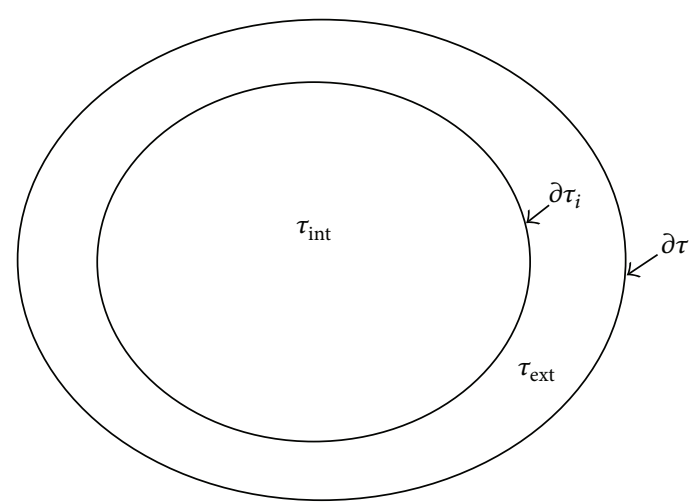

Figure 3: The macroscopic continuum region $\tau$ consisting of the subsystems $\tau_{\text {int }}$ and $\tau_{\text {ext }}$ interacting on the surface $\partial \tau_{i}$ of the continuum velocity jump.

\section{Generalized Thermohydrogravidynamic Shear-Rotational and Classical Shear and Rotational Models of the Earthquake Focal Region}

3.1. The Generalized Thermohydrogravidynamic Shear-Rotational and the Classical Shear (Deformational) Models of the Earthquake Focal Region Based on the Generalized Differential Formulation of the First Law of Thermodynamics. Following the works $[11,12]$, we shall present the foundation of the generalized thermohydrogravidynamic shear-rotational model of the earthquake focal region based on the generalized differential formulation (59) of the first law of thermodynamics. Using the evolution equation (73) of the total mechanical energy of the subsystem $\tau$ (the macroscopic continuum region $\tau$ ) of the Earth, we shall show now that the formation of fractures (modeling by the jumps of the continuum velocity on some surfaces) is related to irreversible dissipation of the macroscopic kinetic energy and the corresponding increase of entropy. We consider at the beginning the analysis of formation of the main line flat fracture (associated with the surface $F_{1}(\tau)$ of the continuum velocity jump) inside the macroscopic continuum region $\tau$ (bounded by the closed surface $\partial \tau$ ). The macroscopic continuum region $\tau$ may be divided into two subsystems $\tau_{1}$ and $\tau_{2}$ by continuing mentally the surface $F_{1}(\tau)$ by means of surface $R_{1}(\tau)$ crossing the surface $\partial \tau$ of the macroscopic region $\tau$. The surface of the subsystem $\tau_{1}$ consists of the surface $(\partial \tau)_{1}$ (which is the part of the surface $\partial \tau)$ and the surfaces $F_{1}(\tau)$ and $R_{1}(\tau)$. The surface of the subsystem $\tau_{2}$ consists of the surface $(\partial \tau)_{2}$ (which is the part of the surface $\partial \tau$ ) and the surfaces $F_{1}(\tau)$ and $R_{1}(\tau)$.

Using the formulation (73), we have the evolution equations for the total mechanical energy of the macroscopic subsystems $\tau_{1}$ and $\tau_{2}$ :

$$
\begin{aligned}
& \frac{d}{d t}\left(K_{\tau_{1}}+\pi_{\tau_{1}}\right) \\
& =\frac{d}{d t} \iiint_{\tau_{1}}\left(\frac{1}{2} \mathbf{v}^{2}+\psi\right) \rho d V \\
& =\iiint_{\tau_{1}} p \operatorname{div} \mathbf{v} d V \\
& +\iiint_{\tau_{1}}\left(\frac{2}{3} \eta-\eta_{v}\right)(\operatorname{div} \mathbf{v})^{2} d V \\
& -\iiint_{\tau_{1}} 2 v\left(e_{i j}\right)^{2} \rho d V \\
& +\iint_{(\partial \tau)_{1}}(\mathbf{v} \cdot(\mathbf{n} \cdot \mathbf{T})) d \Omega_{\mathbf{n}} \\
& +\iint_{F_{1}(\tau)}\left(\mathbf{v}_{1}\left(\tau_{1}\right) \cdot\left(\boldsymbol{\zeta}_{1} \cdot \mathbf{T}\right)\right) d \Sigma_{\zeta_{1}} \\
& +\iint_{R_{1}(\tau)}\left(\mathbf{v}_{1}\left(\tau_{1}\right) \cdot\left(\boldsymbol{\zeta}_{1} \cdot \mathbf{T}\right)\right) d \Sigma_{\zeta_{1}} \\
& +\iiint_{\tau_{1}} \frac{\partial \psi}{\partial t} \rho d V \\
& \frac{d}{d t}\left(K_{\tau_{2}}+\pi_{\tau_{2}}\right) \\
& =\frac{d}{d t} \iiint_{\tau_{2}}\left(\frac{1}{2} \mathbf{v}^{2}+\psi\right) \rho d V \\
& =\iiint_{\tau_{2}} p \operatorname{div} \mathbf{v} d V \\
& +\iiint_{\tau_{2}}\left(\frac{2}{3} \eta-\eta_{v}\right)(\operatorname{div} \mathbf{v})^{2} d V \\
& -\iiint_{\tau_{2}} 2 \nu\left(e_{i j}\right)^{2} \rho d V \\
& +\iint_{(\partial \tau)_{2}}(\mathbf{v} \cdot(\mathbf{n} \cdot \mathbf{T})) d \Omega_{\mathbf{n}} \\
& -\iint_{F_{1}(\tau)}\left(\mathbf{v}_{1}\left(\tau_{2}\right) \cdot\left(\zeta_{1} \cdot \mathbf{T}\right)\right) d \Sigma_{-\zeta_{1}} \\
& -\iint_{R_{1}(\tau)}\left(\mathbf{v}_{1}\left(\tau_{2}\right) \cdot\left(\zeta_{1} \cdot \mathbf{T}\right)\right) d \Sigma_{-\zeta_{1}} \\
& +\iiint_{\tau_{2}} \frac{\partial \psi}{\partial t} \rho d V
\end{aligned}
$$


where $\zeta_{1}$ is the external unit normal vector of the surface (of the subsystem $\tau_{1}$ ) presented by surfaces $F_{1}(\tau)$ and $R_{1}(\tau)$ and $-\zeta_{1}$ is the external unit normal vector of the surface (of the subsystem $\tau_{2}$ ) presented also by surfaces $F_{1}(\tau)$ and $R_{1}(\tau)$. Adding (77) (by using the equality $d \Sigma_{\zeta_{1}}=d \Sigma_{-\zeta_{1}}$ of the elements of area of surfaces $F_{1}(\tau)$ and $\left.R_{1}(\tau)\right)$ we get the evolution equation for the total mechanical energy $\left(K_{\tau}+\pi_{\tau}\right)=$ $\left(K_{\tau_{1}}+K_{\tau_{2}}+\pi_{\tau_{1}}+\pi_{\tau_{2}}\right)$ of the macroscopic region $\tau$ consisting of subsystems $\tau_{1}$ and $\tau_{2}$ interacting on the surface $F_{1}(\tau)$ of the tangential jump of the continuum velocity:

$$
\begin{aligned}
& \frac{d}{d t}\left(K_{\tau}+\pi_{\tau}\right) \\
& =\frac{d}{d t} \iiint_{\tau}\left(\frac{1}{2} \mathbf{v}^{2}+\psi\right) \rho d V \\
& =\iiint_{\tau} p \operatorname{div} \mathbf{v} d V \\
& \quad+\iiint_{\tau}\left(\frac{2}{3} \eta-\eta_{v}\right)(\operatorname{div} \mathbf{v})^{2} d V \\
& \quad-\iiint_{\tau} 2 \nu\left(e_{i j}\right)^{2} \rho d V \\
& \quad+\iint_{\partial \tau}(\mathbf{v} \cdot(\mathbf{n} \cdot \mathbf{T})) d \Omega_{\mathbf{n}} \\
& \quad+\iint_{F_{1}(\tau)}\left(\left(\mathbf{v}_{1}\left(\tau_{1}\right)-\mathbf{v}_{1}\left(\tau_{2}\right)\right) \cdot\left(\zeta_{1} \cdot \mathbf{T}\right)\right) d \Sigma_{\zeta_{1}} \\
& \quad+\iiint_{\tau} \frac{\partial \psi}{\partial t} \rho d V
\end{aligned}
$$

where $\mathbf{v}_{1}\left(\tau_{1}\right)$ is the vector of the continuum velocity on the surface $F_{1}(\tau)$ in the subsystem $\tau_{1}$, and $\mathbf{v}_{1}\left(\tau_{2}\right)$ is the vector of the continuum velocity on the surface $F_{1}(\tau)$ in the subsystem $\tau_{2}$.

The evolution equation (78) takes into account the total mechanical energy $\left(K_{\tau}+\pi_{\tau}\right)$ of the macroscopic region $\tau$ consisting of subsystems $\tau_{1}$ and $\tau_{2}$ interacting on the surface $F_{1}(\tau)$ of the tangential jump of the continuum velocity. The first term in the right-hand side (of (78)) describes the evolution of the total mechanical energy of the macroscopic continuum region $\tau$ due to the continuum reversible compressibility; the second and third terms express the dissipation of the macroscopic kinetic energy by means of the irreversible continuum compressibility and the velocity shear. The forms of three primary terms in the right-hand side (of (78)) are related to the considered model of the compressible viscous Newtonian continuum. The fourth, fifth, and sixth terms in the right-hand side (of (78)) are the universal terms for arbitrary model of continuum characterized by symmetrical stress tensor $\mathbf{T}$. The fourth term expresses the power

$$
W_{\mathrm{np}, \partial \tau}=\frac{\delta A_{\mathrm{np}, \partial \tau}}{d t}=\iint_{\partial \tau}(\mathbf{v} \cdot(\mathbf{n} \cdot \mathbf{T})) d \Omega_{\mathbf{n}}
$$

of external (for the continuum region $\tau$ ) nonpotential stress forces acting on the boundary surface $\partial \tau$ of the macroscopic continuum region $\tau$. The fifth term expresses the power of external (for the continuum region $\tau$ ) forces on different sides of the surface $F_{i}(\tau)$ characterized by the velocity jump during the fracture formation. The sixth term in (78) presents the power of the total mechanical energy added (or lost) as the result of the Newtonian nonstationary gravitational energy influence on the macroscopic continuum region $\tau$ related to variations of the potential $\psi$ of the combined gravitational field in the continuum region $\tau$.

Consider (78) for one continuum velocity jump on the nonstationary surface $F_{1}(\tau)$ during the time interval $(t, t+\Delta t)$. Taking into account the form of the fifth term on the righthand side of the evolution equation (78), we obtained [11, 12] the expression for the work $\delta A_{\mathrm{np}, F_{1}(\tau)}$ (done during the time interval $(t, t+\Delta t)$ by the external (for the continuum region $\tau$ ) nonpotential stress forces acting on different sides of the velocity jump on the surface $\left.F_{1}(\tau)\right)$ :

$$
\begin{aligned}
& \delta A_{\mathrm{np}, F_{1}(\tau)} \\
& \quad=\int_{t}^{t+\Delta t}\left(\iint_{F_{1}(\tau)}\left(\left(\mathbf{v}_{1}\left(\tau_{1}\right)-\mathbf{v}_{1}\left(\tau_{2}\right)\right) \cdot\left(\zeta_{1} \cdot \mathbf{T}\right)\right) d \Sigma_{\zeta_{1}}\right) d t
\end{aligned}
$$

which is reduced to the following expression:

$$
\begin{aligned}
& \delta A_{\mathrm{np}, F_{1}(\tau)} \\
& \quad=\iint_{F_{1}(\tau)}\left(\int_{t}^{t+\Delta t}\left(\mathbf{v}_{1}\left(\tau_{1}\right)-\mathbf{v}_{1}\left(\tau_{2}\right)\right) \cdot\left(\zeta_{1} \cdot \mathbf{T}\right) d t\right) d \Sigma_{\zeta_{1}} .
\end{aligned}
$$

To test the formula (81), let us calculate the energy $\delta A_{\mathrm{np}, \Delta \Sigma}$, which dissipates during formation of the surface dislocation on the small surface $\Delta \Sigma$ during the time interval $(t, t+\Delta t)$. Using the theorem about the average value and integrating the internal integral on time, we obtained from relation $(81)$ for $F_{1}(\tau)=\Delta \Sigma$ the following relation $[11,12]$ :

$$
\begin{aligned}
& \delta A_{\mathrm{np}, \Delta \Sigma} \\
& \quad=\iint_{\Delta \Sigma}\left(\mathbf{w}\left(\zeta_{1}, t+\Delta t\right)-\mathbf{w}\left(-\zeta_{1}, t+\Delta t\right)\right) \cdot\left\langle\left(\zeta_{1} \cdot \mathbf{T}\right)\right\rangle d \Sigma_{\zeta_{1}},
\end{aligned}
$$

where $\left\langle\left(\zeta_{1} \cdot \mathbf{T}\right)\right\rangle$ is the average value of the stress vector for the element of area $d \Sigma_{\zeta_{1}}$ of the two-side surface $\Delta \Sigma$, and $\mathbf{w}\left(\zeta_{1}, t+\right.$ $\Delta t)$ and $\mathbf{w}\left(-\zeta_{1}, t+\Delta t\right)$ are the vectors of the continuum displacement on different sides of the element of area $d \Sigma_{\zeta_{1}}$ of the two-side surface $\Delta \Sigma$ in the points characterized by normal unit vectors $\zeta_{1}$ and $-\zeta_{1}$. Using the obvious expression for "linear" time average $\left\langle\left(\zeta_{1} \cdot \mathbf{T}\right)\right\rangle$

$$
\left\langle\left(\zeta_{1} \cdot \mathbf{T}\right)\right\rangle=\frac{1}{2}\left(\mathbf{p}\left(\zeta_{1}, t\right)-\mathbf{p}\left(-\zeta_{1}, t+\Delta t\right)\right)
$$

as the arithmetical average of the values of the stress vectors $\mathbf{p}$ on the different sides from the surface of the jump of the continuum velocity, we obtained $[11,12]$ the expression for 
the elementary work of the external nonpotential stress forces on the two-side surface $\Delta \Sigma$ of dislocation:

$$
\begin{array}{r}
\delta A_{\mathrm{np}, \Delta \Sigma} \\
=\frac{1}{2} \iint_{\Delta \Sigma}\left(\mathbf{w}\left(\zeta_{1}, t+\Delta t\right)-\mathbf{w}\left(-\zeta_{1}, t+\Delta t\right)\right) \\
\cdot\left(\mathbf{p}\left(\zeta_{1}, t\right)+\mathbf{p}\left(-\zeta_{1}, t+\Delta t\right)\right) d \Sigma_{\zeta_{1}} .
\end{array}
$$

This expression was obtained [44] in the frame of the classical linear approach to formation of surface dislocations in rigid compressible continuum on the small area of surface $\Delta \Sigma$. It is clear that the assumption (83) is valid only for weak tangential jumps of the continuum displacement. Consequently, we can consider the expression (80) as the natural nonlinear generalization of expression (84) for arbitrary surface $F_{1}(\tau)$ of dislocation and for strong tangential jumps of the continuum displacement on the surface $F_{1}(\tau)$ of dislocation. The work (80) of the external (for the continuum region $\tau$ ) nonpotential stress forces should be negative. The sufficient energy $\delta E_{d, F_{1}(\tau)}$ needed for formation of the surface $F_{1}(\tau)$ of dislocation is equal to the work of the internal forces in the macroscopic continuum region $\tau$. The energy $\delta E_{d, F_{1}(\tau)}$ should be positive and equal to the expression (80) with the sign "-":

$$
\begin{aligned}
& \delta E_{d, F_{1}(\tau)} \\
& =-\delta A_{\mathrm{np}, F_{1}(\tau)} \\
& =-\int_{t}^{t+\Delta t}\left(\iint_{F_{1}(\tau)}\left(\left(\mathbf{v}_{\mathbf{1}}\left(\tau_{1}\right)-\mathbf{v}_{\mathbf{1}}\left(\tau_{2}\right)\right) \cdot\left(\boldsymbol{\zeta}_{1} \cdot \mathbf{T}\right)\right) d \Sigma_{\zeta_{1}}\right) d t \\
& >0
\end{aligned}
$$

The formulae (80), (84), and (85) are obtained (taking into account the generalized differential formulation (50) of the first law of thermodynamics) for the model of continuum characterized by an arbitrary symmetrical stress tensor $\mathbf{T}$.

The macroscopic internal shear kinetic energy $\left(K_{s}\right)_{\tau_{1}}$ (of the subsystem $\tau_{1}$ ), the macroscopic internal rotational kinetic energy $\left(K_{r}\right)_{\tau_{1}}$ (of the subsystem $\tau_{1}$ ), and the macroscopic kinetic energy of shear-rotational coupling $\left(K_{s, r}^{\text {coup }}\right)_{\tau_{1}}$ (of the subsystem $\tau_{1}$ ) are the significant components of the macroscopic internal shear-rotational kinetic energy $\left(K_{s-r}\right)_{\tau_{1}}[11,12$, 24, 31]:

$$
\left(K_{s-r}\right)_{\tau_{1}}=\left(K_{r}\right)_{\tau_{1}}+\left(K_{s}\right)_{\tau_{1}}+\left(K_{s, r}^{\text {coup }}\right)_{\tau_{1}}
$$

taken into account (along with the classical internal thermal energy $U_{\tau_{1}}$ of the macroscopic continuum region $\tau_{1}$, the macroscopic potential energy $\pi_{\tau_{1}}$ of the macroscopic continuum region $\tau_{1}$, and the macroscopic translational kinetic energy $\left(K_{t}\right)_{\tau_{1}}=(1 / 2) m_{\tau_{1}}\left(\mathbf{V}_{c}\right)_{\tau_{1}}^{2}$ of the continuum region $\tau_{1}$ (of a mass $m_{\tau_{1}}$ ) moving as a whole at speed equal to the speed $\left(\mathbf{V}_{c}\right)_{\tau_{1}}$ of the center of mass of the continuum region $\left.\tau_{1}\right)$ in the generalized differential formulation (50) of the first law of thermodynamics for the macroscopic continuum region $\tau_{1}$.

The macroscopic internal shear kinetic energy $\left(K_{s}\right)_{\tau_{2}}$ (of the subsystem $\tau_{2}$ ), the macroscopic internal rotational kinetic energy $\left(K_{r}\right)_{\tau_{2}}$, and the macroscopic kinetic energy of shearrotational coupling $\left(K_{s, r}^{\text {coup }}\right)_{\tau_{2}}$ are the significant components of the macroscopic internal shear-rotational kinetic energy $\left(K_{s-r}\right)_{\tau_{2}}[11,12,24,31]$ :

$$
\left(K_{s-r}\right)_{\tau_{2}}=\left(K_{r}\right)_{\tau_{2}}+\left(K_{s}\right)_{\tau_{2}}+\left(K_{s, r}^{\text {coup }}\right)_{\tau_{2}}
$$

taken into account (along with the classical internal thermal energy $U_{\tau_{2}}$, the macroscopic potential energy $\pi_{\tau_{2}}$, and the macroscopic translational kinetic energy $\left(K_{t}\right)_{\tau_{2}} \stackrel{\tau_{2}}{=}$ $(1 / 2) m_{\tau_{2}}\left(\mathbf{V}_{c}\right)_{\tau_{2}}^{2}$ of the continuum region $\tau_{2}$ (of a mass $m_{\tau_{2}}$ ) moving as a whole at the speed $\left(\mathbf{V}_{c}\right)_{\tau_{2}}$ of the center of mass of the continuum region $\tau_{2}$ ) in the generalized differential formulation (50) of the first law of thermodynamics for the macroscopic continuum region $\tau_{2}$.

The macroscopic internal shear kinetic energy $\left(K_{s}\right)_{\tau_{1}}$ (of the subsystem $\tau_{1}$ ), the macroscopic internal rotational kinetic energy $\left(K_{r}\right)_{\tau_{1}}$, the macroscopic kinetic energy of shear-rotational coupling $\left(K_{s, r}^{\text {coup }}\right)_{\tau_{1}}$, the macroscopic translational kinetic energy $\left(K_{t}\right)_{\tau_{1}}=(1 / 2) m_{\tau_{1}}\left(\mathbf{V}_{c}\right)_{\tau_{1}}^{2}$, the macroscopic potential energy $\boldsymbol{\pi}_{\tau_{1}}$, the macroscopic internal shear kinetic energy $\left(K_{s}\right)_{\tau_{2}}$ (of the subsystem $\tau_{2}$ ), the macroscopic internal rotational kinetic energy $\left(K_{r}\right)_{\tau_{2}}$, the macroscopic kinetic energy of shear-rotational coupling $\left(K_{s, r}^{\text {coup }}\right)_{\tau_{2}}$, the macroscopic translational kinetic energy $\left(K_{t}\right)_{\tau_{2}}=(1 / 2) m_{\tau_{2}}\left(\mathbf{V}_{c}\right)_{\tau_{2}}^{2}$, and the macroscopic potential energy $\boldsymbol{\pi}_{\tau_{2}}$ are the significant energy components taken into account in the presented thermohydrogravidynamic shear-rotational model described by the evolution equation (78) for the total mechanical energy $\left(K_{\tau}+\right.$ $\pi_{\tau}$ ) of the macroscopic region $\tau$ consisting of interacting subsystems $\tau_{1}$ and $\tau_{2}$.

3.2. The Rotational Model of the Earthquake Focal Region Based on the Generalized Differential Formulation of the First Law of Thermodynamics. Following the works [11, 12], we shall present the foundation of the rotational model [2] of the earthquake focal region for the seismic zone of the Pacific Ring. It was noted [2] that the studies of the dislocation models of the focal regions of strong earthquakes showed the bad correspondence with the model of flat endless dislocation in the uniform continuum [45-47]. The analysis [2] showed that the conditions exist to realize the rotational mechanism related to the rotation of the geoblocks by means of the stress forces related to the Earth rotation in the vicinity of the seismic zone of the Pacific Ring. It was noted [2] that the rotational mechanism can be more real compared to the conventional mechanism related to the formation of the main line flat fracture inside the focal region.

Let us consider the energy thermodynamic analysis of the rotational mechanism [2] of the earthquake focal region, related to formation of the circular continuum velocity jump revealed in the form of circular dislocation after relaxation of the seismic process in the earthquake focal region. The developed and tested (in this section) mathematical formalism of description of the main line flat fracture may be generalized on the closed surfaces of the continuum velocity jumps. Following the rotational model [2] of the earthquake focal 
region, we consider the separate geoblock $\tau_{\text {int }}$ of the seismic zone. If the external influences of the nonstationary gravitational forces (on the geoblock $\tau_{\text {int }}$ ) and the nonpotential stress forces (on the boundary $\partial \tau_{i}$ of the geoblock $\tau_{\text {int }}$ ) exceed the certain critical value then the geoblock may rotate and slip relative to the surrounding fine plastic layer (subsystem) $\tau_{\text {ext }}$ with the tangential continuum velocity jump on the boundary surface $\partial \tau_{i}$ of the geoblock $\tau_{\text {int }}$. We assume that fine plastic layer (subsystem) $\tau_{\text {ext }}$ is limited by external surface $\partial \tau$ of the considered thermodynamic system $\tau$ consisting of the macroscopic subsystems $\tau_{\text {int }}$ and $\tau_{\text {ext }}$.

Using the evolution equation (73) of the total mechanical energy of the subsystem $\tau$, we obtained $[11,12]$ the evolution equations for the total mechanical energy of the macroscopic subsystems $\tau_{\text {int }}$ and $\tau_{\text {ext }}$ :

$$
\begin{aligned}
& \frac{d}{d t}\left(K_{\tau_{\text {int }}}+\pi_{\tau_{\text {int }}}\right) \\
& =\frac{d}{d t} \iiint_{\tau_{\text {int }}}\left(\frac{1}{2} \mathbf{v}^{2}+\psi\right) \rho d V \\
& =\iiint_{\tau_{\text {int }}} p \operatorname{div} \mathbf{v} d V \\
& +\iiint_{\tau_{\text {int }}}\left(\frac{2}{3} \eta-\eta_{v}\right)(\operatorname{div} \mathbf{v})^{2} d V \\
& -\iiint_{\tau_{\text {int }}} 2 \nu\left(e_{i j}\right)^{2} \rho d V \\
& +\iint_{\partial \tau_{i}}\left(\mathbf{v}_{\text {int }}\left(\partial \tau_{i}\right) \cdot(\mathbf{m} \cdot \mathbf{T})\right) d \Sigma_{\mathbf{m}} \\
& +\iiint_{\tau_{\text {int }}} \frac{\partial \psi}{\partial t} \rho d V \\
& \frac{d}{d t}\left(K_{\tau_{\text {ext }}}+\pi_{\tau_{\text {ext }}}\right) \\
& =\frac{d}{d t} \iiint_{\tau_{\mathrm{ext}}}\left(\frac{1}{2} \mathbf{v}^{2}+\psi\right) \rho d V \\
& =\iiint_{\tau_{\text {ext }}} p \operatorname{div} \mathbf{v} d V \\
& +\iiint_{\tau_{\mathrm{ext}}}\left(\frac{2}{3} \eta-\eta_{v}\right)(\operatorname{div} \mathbf{v})^{2} d V \\
& -\iiint_{\tau_{\text {ext }}} 2 \nu\left(e_{i j}\right)^{2} \rho d V \\
& +\iint_{\partial \tau}(\mathbf{v} \cdot(\mathbf{n} \cdot \mathbf{T})) d \Omega_{\mathbf{n}} \\
& -\iint_{\partial \tau_{i}}\left(\mathbf{v}_{\text {ext }}\left(\partial \tau_{i}\right) \cdot(\mathbf{m} \cdot \mathbf{T})\right) d \Sigma_{-\mathbf{m}} \\
& +\iiint_{\tau_{\text {ext }}} \frac{\partial \psi}{\partial t} \rho d V
\end{aligned}
$$

where $\mathbf{m}$ is the external unit normal vector of the surface $\partial \tau_{i}$ of the subsystem $\tau_{\text {int }},-\mathbf{m}$ is the internal unit normal vector of the surface $\partial \tau_{i}, \mathbf{n}$ is the external unit normal vector of the surface $\partial \tau, \mathbf{v}_{\text {int }}\left(\partial \tau_{i}\right)$ are the velocities vectors on the inner side of the surface $\partial \tau_{i}$ in the subsystem $\tau_{\text {int }}$, and $\mathbf{v}_{\text {ext }}\left(\partial \tau_{i}\right)$ are the velocities vectors on the outer side of the surface $\partial \tau_{i}$ in the subsystem $\tau_{\text {ext }}$.

Adding the evolution equation (88) and using the condition of equality $d \Sigma_{\mathbf{m}}=d \Sigma_{-\mathbf{m}}$ of the area elements of the surface $\partial \tau_{i}$, we get the evolution equation for the total mechanical energy of the macroscopic continuum region $\tau$ consisting of the subsystems $\tau_{\text {int }}$ and $\tau_{\text {ext }}$ interacting on the surface $\partial \tau_{i}$ of the continuum velocity jump:

$$
\begin{aligned}
& \frac{d}{d t}\left(K_{\tau}+\pi_{\tau}\right) \\
& =\frac{d}{d t} \iiint_{\tau}\left(\frac{1}{2} \mathbf{v}^{2}+\psi\right) \rho d V \\
& =\iiint_{\tau} p \operatorname{div} \mathbf{v} d V \\
& \quad+\iiint_{\tau}\left(\frac{2}{3} \eta-\eta_{v}\right)(\operatorname{div} \mathbf{v})^{2} d V \\
& \quad-\iiint_{\tau} 2 v\left(e_{i j}\right)^{2} \rho d V \\
& \quad+\iint_{\partial \tau}(\mathbf{v} \cdot(\mathbf{n} \cdot \mathbf{T})) d \Omega_{\mathbf{n}} \\
& \quad+\iint_{\partial \tau_{i}}\left(\left(\mathbf{v}_{\mathrm{int}}\left(\partial \tau_{i}\right)-\mathbf{v}_{\mathrm{ext}}\left(\partial \tau_{i}\right)\right) \cdot(\mathbf{m} \cdot \mathbf{T})\right) d \Sigma_{\mathbf{m}} \\
& \quad+\iiint_{\tau} \frac{\partial \psi}{\partial t} \rho d V .
\end{aligned}
$$

Equation (89) is analogous to (78). The energy needed for formation of the continuum velocities jumps (on the surfaces $F_{1}(\tau)$ and $\left.\partial \tau_{i}\right)$ is related to the penultimate terms in the righthand sides of (78) and (89). Similar to expression (85), we have the expression for the sufficient energy $\delta E_{d, \partial \tau_{i}}$ needed to rotate and slip (for the subsystem $\tau_{\text {int }}$ ) during the time interval $(t, t+\Delta t)$ relative to the surrounding fine plastic layer (subsystem) $\tau_{\text {ext }}$ (with the tangential continuum velocity jump $\left(\mathbf{v}_{\text {int }}\left(\partial \tau_{i}\right)-\mathbf{v}_{\text {ext }}\left(\partial \tau_{i}\right)\right)$ on the boundary surface $\partial \tau_{i}$ of the geoblock $\left.\tau_{\text {int }}\right)$ :

$$
\begin{array}{r}
\delta E_{d, \partial \tau_{i}}=-\delta A_{\mathrm{np}, \partial \tau_{\mathrm{i}}} \\
=-\int_{t}^{t+\Delta t}\left(\int \int _ { \partial \tau _ { i } } \left(\left(\mathbf{v}_{\mathrm{int}}\left(\partial \tau_{i}\right)-\mathbf{v}_{\mathrm{ext}}\left(\partial \tau_{i}\right)\right)\right.\right. \\
\left.\cdot(\mathbf{m} \cdot \mathbf{T})) d \Sigma_{\mathbf{m}}\right) d t>0 .
\end{array}
$$

Taking into account the information [2] that the critical continuum stresses (required for rotation of the geoblock $\tau_{\text {int }}$ weakly coupled with the surrounding plastic layer $\tau_{\text {ext }}$ ) are less than the critical continuum stresses required to split the mountain rock by forming the main line flat fracture, we concluded $[11,12]$ that the required energy $\delta E_{d, \partial \tau_{i}}$ (given by the expression (90)) is less than the required energy $\delta E_{d, F_{1}(\tau)}$ 
(given by the expression (85)) if the displacements of the rock continuum on different sides of the analyzed different jumps of the continuum displacements (the closed dislocation and the main line flat fracture) have the same order of magnitude and the ratio of the surfaces area of the closed dislocation to the surfaces area of the main line flat fracture does not exceed 10.

This thermodynamic energy consideration showed [11, 12] the preferable realization of the rotational motion of the geoblock $\tau_{\text {int }}$ (under condition that exists the surrounding plastic layer around the geoblock $\tau_{\text {int }}$ ) as compared with formation of the of the main line flat fracture inside the geoblock $\tau_{\text {int }}$. This result explains the rotational motions of the geoblocks in the seismic zone of the Pacific Ring [2] and the vortical structures of the lithosphere of Earth [48] and the lithospheres of the planets [49] of the Solar System.

Using of the generalized differential formulation (50) of the first law of thermodynamics for the macroscopic continuum region $\tau$ of the quasi-uniform medium of the Earth's crust characterized by practically constant viscosity, we obtained $[11,12]$ the thermodynamic foundation of the classical deformational (shear) model [1] of the earthquake focal region for the quasi-uniform medium of the Earth's crust characterized by practically constant viscosity.

\section{Fundamentals of the Cosmic Geophysics}

\subsection{The Energy Gravitational Influences on the Earth of the Planets of the Solar System}

4.1.1. The Instantaneous Energy Gravitational Influences on the Earth of the Planets of the Solar System in the Approximation of the Elliptical Orbits of the Planets. We shall consider the movements of the Earth $\tau_{3}$ and the outer (or inner) planet $\tau_{i}$ in the ecliptic plane $X Z$ (see Figure 4) around the Sun $\tau_{0,0}$ in the approximation of the elliptical orbits of the planets. The mass center $C_{3}$ of the Earth $\tau_{3}$, the mass center $O$ of the Sun, and the mass center $C_{i}$ of the inner $(i=1,2)$ and the outer $(i=4,5,6,7,8,9)$ planet $\tau_{i}$ are located on the direct coordinate axis $X$ at a certain initial time moment $t=0$ characterized by the minimal distance between the mass center $C_{i}$ of the inner $(i=1,2)$ and the outer $(i=4,5,6$, $7,8,9)$ planet $\tau_{i}$ and the mass center $C_{3}$ of the Earth $\tau_{3}$. The fixed mass center $O$ of the Sun is considered as the right focus of the elliptical orbits of the inner $(i=1,2)$ planet $\tau_{i}$, the outer $(i=4,5,6,7,8,9)$ planet $\tau_{i}$, and the Earth $\tau_{3}$.

We have the following relations:

$$
\begin{gathered}
r_{i}\left(\varphi_{i}(t)\right)=\frac{p_{i}}{\left(1+e_{i} \cos \varphi_{i}(t)\right)}, \quad(i=1,2,4,5,6,7,8,9) \\
r_{3}\left(\varphi_{3}(t)\right)=\frac{p_{3}}{\left(1+e_{3} \cos \varphi_{3}(t)\right)}
\end{gathered}
$$

for the distance $r_{i}\left(\varphi_{i}(t)\right)$ between the mass center $O$ of the Sun and the mass center $C_{i}$ of the inner $(i=1,2)$ or the outer $(i=4,5,6,7,8,9)$ planet $\tau_{i}$ and for the distance $r_{3}\left(\varphi_{3}(t)\right)$

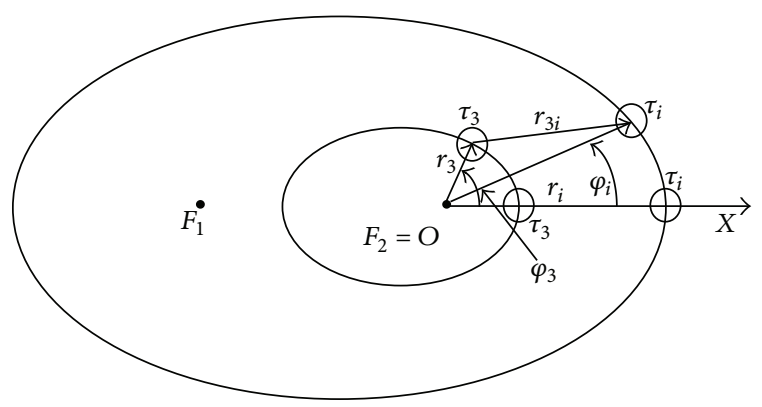

FIGURE 4: The geometric sketch of circulation of the outer planet $\tau_{i}$ (Mars or Jupiter, Saturn, Uranus, Neptune, and Pluto) and the Earth $\tau_{3}$ around the mass center $O$ of the Sun.

between the mass center $O$ of the Sun and the mass center $C_{3}$ of the Earth $\tau_{3}$. Here $p_{i}$ and $e_{i}$ are the focal parameters and the eccentricity, respectively, of the elliptical orbit of the inner $(i=1,2)$ and the outer $(i=4,5,6,7,8,9)$ planet $\tau_{i}$ and $p_{3}$ and $e_{3}$ are the focal parameters and the eccentricity, respectively, of the elliptical Earth's orbit. We have $\varphi_{i}(0)=0$ $(i=1,2,3,4,5,6,7,8,9)$ for the initial time moment $t=0$.

We shall consider the gravitational potential $\psi_{3 i}\left(C_{3}\right.$, int, ext, $\left.t, r_{3}\left(\varphi_{3}(t)\right)\right)$ created by the inner $(i=1,2)$ or the outer $(i=4,5,6,7,8,9)$ planet $\tau_{i}$ in the mass center $C_{3}$ (of the Earth $\left.\tau_{3}\right)$ :

$$
\psi_{3 i}\left(C_{3}, \text { int, ext, } t, r_{3}\left(\varphi_{3}(t)\right)\right)=-\gamma \frac{M_{i}}{r_{3 i}\left(C_{3}, C_{i}, t\right)},
$$

where $r_{3 i}\left(C_{3}, C_{i}, t\right)$ is the distance between the mass center $C_{3}$ of the Earth $\tau_{3}$ and the mass center $C_{i}$ of the inner $(i=1,2)$ or the outer $(i=4,5,6,7,8,9)$ planet $\tau_{i}$. We find the distance $r_{3 i}\left(C_{3}, C_{i}, t\right)$ for the outer $(i=4,5,6,7,8,9)$ planet $\tau_{i}$ from the following relation:

$$
\begin{aligned}
\left(r_{3 i}(\right. & \left.\left(C_{3}, C_{i}, t\right)\right)^{2} \\
= & \left(r_{3}\left(\varphi_{3}(t)\right)\right)^{2}+\left(r_{i}\left(\varphi_{i}(t)\right)\right)^{2} \\
& \quad-r_{3}\left(\varphi_{3}(t)\right) r_{i}\left(\varphi_{i}(t)\right) \cos \left(\varphi_{3}(t)-\varphi_{i}(t)\right),
\end{aligned}
$$

which is valid also for the inner planet $\tau_{i}(i=1,2)$. The relation (93) can be rewritten as follows:

$$
\begin{aligned}
\psi_{3 i}\left(C_{3}, \text { int, ext, } t, r_{3}\left(\varphi_{3}(t)\right)\right) & \\
= & -\left(\gamma M_{i}\right) \\
& \times\left(\left(r_{3}\left(\varphi_{3}\right)\right)^{2}+\left(r_{i}\left(\varphi_{i}(t)\right)\right)^{2}\right. \\
& \left.\quad-2 r_{3}\left(\varphi_{3}\right) r_{i}\left(\varphi_{i}(t)\right) \cos \left(\varphi_{3}-\varphi_{i}(t)\right)\right)^{-1 / 2} .
\end{aligned}
$$


We obtained $[25,26]$ the expression for the partial derivative of the gravitational potential (95):

$$
\begin{aligned}
\frac{\partial}{\partial t} \psi_{3 i}\left(C_{3}, \text { int, ext, } t, r_{3}\left(\varphi_{3}(t)\right)\right) & \left(\gamma M_{i} r_{i}\left(\varphi_{i}(t)\right) \frac{d \varphi_{i}(t)}{d t}\right) \\
\times & \left(\left(r_{3}\left(\varphi_{3}(t)\right)\right)^{2}+\left(r_{i}\left(\varphi_{i}(t)\right)\right)^{2}\right. \\
& \left.\quad-2 r_{3}\left(\varphi_{3}(t)\right) r_{i}\left(\varphi_{i}(t)\right) \cos \left(\varphi_{3}(t)-\varphi_{i}(t)\right)\right)^{-3 / 2} \\
\times & \left\{r_{3}\left(\varphi_{3}(t)\right) \sin \left(\varphi_{i}(t)-\varphi_{3}(t)\right)+\frac{e_{i} r_{i}\left(\varphi_{i}(t)\right) \sin \varphi_{i}(t)}{\left(1+e_{i} \cos \varphi_{i}(t)\right)}\right. \\
& \left.-\frac{e_{i} r_{3}\left(\varphi_{3}(t)\right) \sin \varphi_{i}(t) \cos \left(\varphi_{i}(t)-\varphi_{3}(t)\right)}{\left(1+e_{i} \cos \varphi_{i}(t)\right)}\right\},
\end{aligned}
$$

where the distances $r_{i}\left(\varphi_{i}(t)\right)$ and $r_{3}\left(\varphi_{3}(t)\right)$ are given by the relations (91) and (92), respectively. [12]:

The expression (96) is reduced to the following expression

$$
\begin{aligned}
& \frac{\partial}{\partial t} \psi_{3 i}\left(C_{3}, \text { int }\right) \\
& \quad=\frac{\partial}{\partial t} \psi_{3 i}\left(C_{3}, \text { ext }\right) \\
& =\frac{\gamma M_{i} R_{\mathrm{O} 3} R_{\mathrm{O} i} \omega_{i} \sin \left(\omega_{i}-\omega_{3}\right) t}{\left[R_{\mathrm{O} 3}^{2}+R_{\mathrm{O} i}^{2}-2 R_{\mathrm{O} 3} R_{\mathrm{O} i} \cos \left(\omega_{i}-\omega_{3}\right) t\right]^{3 / 2}}
\end{aligned}
$$

under the following conditions: $e_{i}=0, e_{3}=0$, and $d \varphi_{i}(t) / d t=\omega_{i}$ corresponding to the circular orbits of the planet $\tau_{i}(i=1,2,4,5,6,7,8,9)$ and the Earth $\tau_{3}$.

The first term in the figured brackets of the expression (96) gives the principal contribution to the partial derivative $(\partial / \partial t) \psi_{3 i}\left(C_{3}\right.$, int, ext, $\left.t, r_{3}\left(\varphi_{3}(t)\right)\right)$. The expression (96) contains the additional two small terms (vanishing at $e_{i} \rightarrow 0$ and $e_{3} \rightarrow 0$ ) related to the eccentricities $e_{i}$ and $e_{3}$ of the elliptical orbits of the planet $\tau_{i}(i=1,2,4,5,6,7,8,9)$ and the Earth $\tau_{3}$, respectively.

The combined maximal contribution of these additional two terms is of the order

$$
O\left(e_{i}, e_{3}\right)\left(\max \frac{\partial}{\partial t} \psi_{3 i}\left(C_{3}, \text { int }\right)\right)
$$

for the inner $(i=1,2)$ planet $\tau_{i}$ and of the order

$$
O\left(e_{i}, e_{3}\right)\left(\max \frac{\partial}{\partial t} \psi_{3 i}\left(C_{3}, \text { ext }\right)\right)
$$

for the outer $(i=4,5,6,7,8,9)$ planet $\tau_{i}$. Consequently, the contribution of the first term in the figured brackets of the expression (96) is $O\left(1 / e_{i}, 1 / e_{3}\right)$ times larger than the contribution of the additional two new terms related to the eccentricities $e_{i}$ and $e_{3}$. Using the maximal eccentricity $e_{1}=0.206$ of Mercury's orbit, we have that the contribution of the first term in the figured brackets of the expression (96) is approximately 5 times larger than the contribution of the additional two new terms (in the figured brackets of the expression (96))

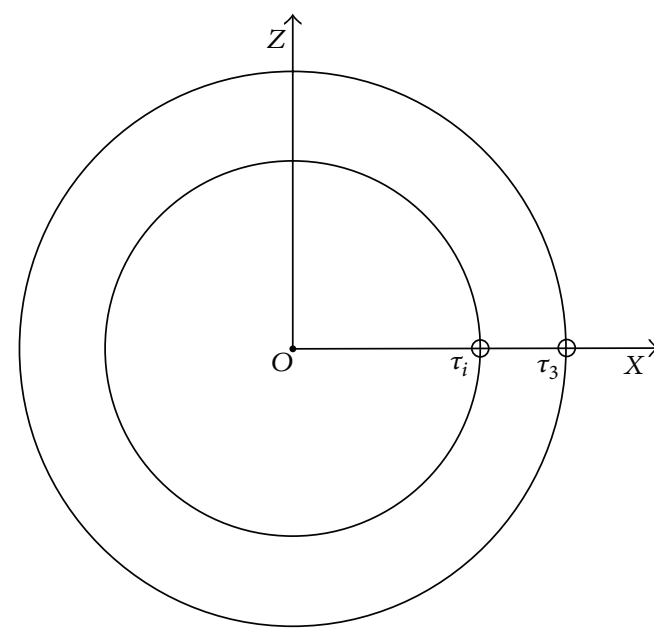

FIGURE 5: The initial $(t=0)$ planetary configuration characterized by the opposition of the inner planet $\tau_{i}$ (Mercury or Venus) and the Earth $\tau_{3}$.

related to the eccentricities $e_{1}=0.206$ and $e_{3}=0.017$ of the elliptical orbits of Mercury $\tau_{1}$ and the Earth $\tau_{3}$, respectively. We have that the first term (in the figured brackets of the expression (96)) is significantly larger than the contribution of the additional two new terms for the other planets (of the Solar System) having the small eccentricities of the elliptical orbits.

Thus, the obtained $[12,25,26]$ evaluation (97) (for the circular orbits of the planets) of the relative maximal energy gravitational influences on the Earth (of the planets of the Solar System) may be considered as the first sound approximation for the evaluation of the relative maximal energy gravitational influences of the inner $(i=1,2)$ and the outer $(i=4,5,6,7,8,9)$ planets on the Earth.

4.1.2. The Evaluation of the Relative Maximal Planetary Instantaneous Energy Gravitational Influences on the Earth in the Approximation of the Circular Orbits of the Planets of the Solar System. Following the monograph [12], we shall consider the movement of the Earth $\tau_{3}$ and the inner planet $\tau_{i}$ around the Sun $\tau_{0,0}$ in the first approximation of the circular orbits of the planets. The planets revolve in the ecliptic plane $X Z$ (see Figures 5 and 6). The mass center of the Sun is located at the fixed point $O$ of the origin of the coordinate system. The mass center $C_{3}$ of the Earth, the mass center $O$ of the Sun, and the mass center $C_{i}$ of the inner planet $\tau_{i}$ are located on the direct coordinate axis $X$ at a certain initial time moment $t=0$ characterized by the minimal distance between the Earth and the inner planet $\tau_{i}$ (see Figure 5 ).

We have the following expressions for the angles $\varphi_{i}$ and $\varphi_{3}$

$$
\begin{gathered}
\varphi_{i}(t)=\omega_{i} t=\frac{2 \pi}{T_{i}} t, \\
\varphi_{3}(t)=\omega_{3} t=\frac{2 \pi}{T_{3}} t,
\end{gathered}
$$

which describe the positions of the mass centers of the planet $\tau_{i}$ and the Earth $\tau_{3}$ during the time $t$. 


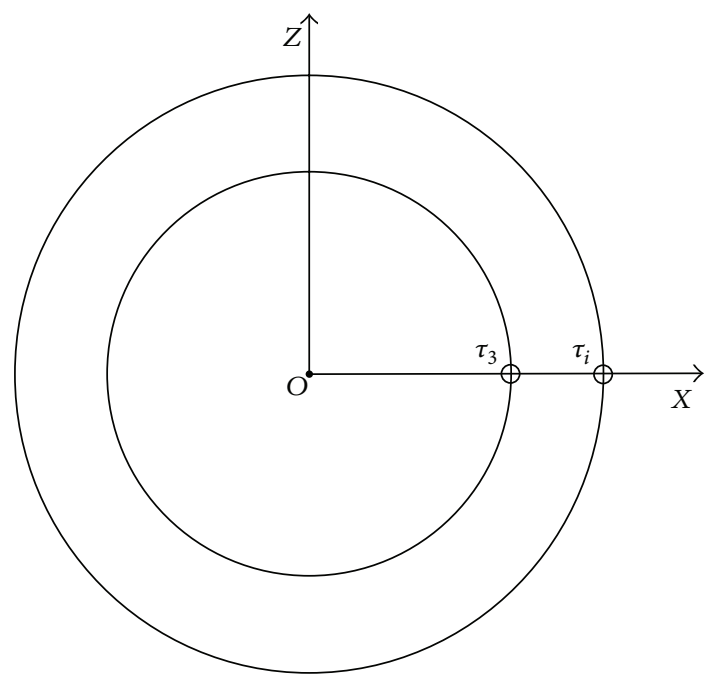

FIGURE 6: The initial planetary configuration of the outer planet $\tau_{i}$ (Mars or Jupiter, Saturn, Uranus, Neptune, and Pluto) and the Earth $\tau_{3}$.

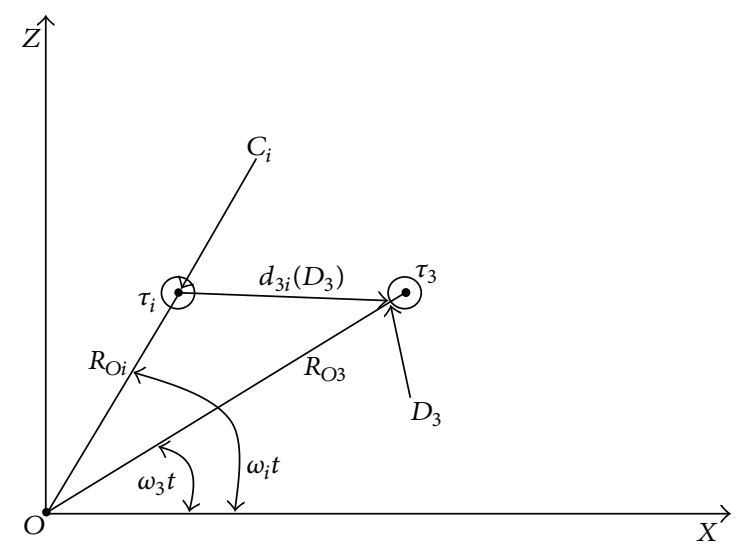

Figure 7: The geometric sketch of circulations of the Earth $\tau_{3}$ and the inner planet $\tau_{i}$ (Mercury or Venus) around the mass center $O$ of the Sun.

We shall consider the gravitational potential $\psi_{3 i}\left(C_{3}\right.$, int $)$

$$
\psi_{3 i}\left(C_{3}, \text { int }\right)=-\gamma \frac{M_{i}}{d_{3 i}\left(C_{3}\right)}
$$

created by the inner planet $\tau_{i}$ in the mass center $C_{3}$ of the Earth. We have the distance $d_{3 i}\left(C_{3}\right)$ between the mass center $C_{i}$ of the inner planet $\tau_{i}$ and the mass center $C_{3}$ of the Earth (see Figure 7):

$$
d_{3 i}^{2}\left(C_{3}\right)=R_{\mathrm{O} 3}^{2}+R_{\mathrm{O} i}^{2}-2 R_{\mathrm{O} 3} R_{\mathrm{O} i} \cos \left(\omega_{i} t-\varphi_{3}\right) .
$$

The relation (101) can be rewritten as follows [12]:

$$
\psi_{3 i}\left(C_{3}, \text { int }\right)=-\frac{\gamma M_{i}}{\sqrt{R_{\mathrm{O} 3}^{2}+R_{\mathrm{O} i}^{2}-2 R_{\mathrm{O} 3} R_{\mathrm{O} i} \cos \left(\omega_{i} t-\varphi_{3}\right)}} .
$$

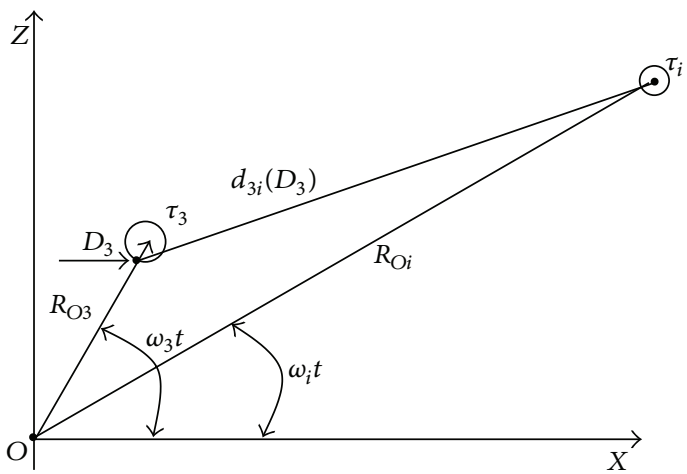

FIGURE 8: The geometric sketch of circulation of the outer planet $\tau_{i}$ (Mars or Jupiter, Saturn, Uranus, Neptune, and Pluto) and Earth $\tau_{3}$ around the mass center $O$ of the Sun.

We derived [12] the expression for the partial derivative $(\partial / \partial t) \psi_{3 i}\left(C_{3}\right.$, int $)$ :

$$
\begin{aligned}
& \frac{\partial}{\partial t} \psi_{3 i}\left(C_{3}, \text { int }\right) \\
& \quad=\frac{\gamma M_{i} R_{\mathrm{O} 3} R_{\mathrm{O} i} \omega_{i} \sin \left(\omega_{i}-\omega_{3}\right) t}{\left[R_{\mathrm{O} 3}^{2}+R_{\mathrm{O} i}^{2}-2 R_{\mathrm{O} 3} R_{\mathrm{O} i} \cos \left(\omega_{i}-\omega_{3}\right) t\right]^{3 / 2}},
\end{aligned}
$$

which is reduced to zero for the time moments $t_{n}^{*}(i, 3)=$ $(1 / 2)\left(T_{i} T_{3} /\left(T_{3}-T_{i}\right)\right) n$ (for $\left.i=1,2 ; n=0,1,2, \ldots\right)$, when the mass centers of the Sun, the Earth, and the inner planet $\tau_{i}$ are located on the direct line.

We have (for configuration of the Earth and the outer planet $\tau_{i}$ shown on Figure 8$)$ the distance $d_{i 3}\left(C_{3}\right)$ between the mass center $C_{i}$ of the planet $\tau_{i}$ and the mass center $C_{3}$ of the Earth:

$$
d_{3 i}^{2}\left(C_{3}\right)=R_{\mathrm{O} 3}^{2}+R_{\mathrm{O} i}^{2}-2 R_{\mathrm{O} 3} R_{\mathrm{O} i} \cos \left(\omega_{3}-\omega_{i}\right) t .
$$

The gravitational potential created by the outer planet $\tau_{i}$ at the point $C_{3}$ (for configuration shown on Figure 8 ) is given by the following expression:

$$
\begin{aligned}
\psi_{3 i}\left(C_{3}, \text { ext }\right) & =-\frac{\gamma M_{i}}{d_{3 i}\left(C_{3}\right)} \\
& =-\frac{\gamma M_{i}}{\sqrt{R_{\mathrm{O} 3}^{2}+R_{\mathrm{O} i}^{2}-2 R_{\mathrm{O} 3} R_{\mathrm{O} i} \cos \left(\varphi_{3}-\omega_{i} t\right)}} .
\end{aligned}
$$

We derived [12] the expression of the partial derivative $(\partial / \partial t) \psi_{3 i}\left(C_{3}\right.$, ext) for the expression (106):

$$
\begin{aligned}
& \frac{\partial}{\partial t} \psi_{3 i}\left(C_{3}, \text { ext }\right) \\
& \quad=-\frac{\gamma M_{i} R_{\mathrm{O} 3} R_{\mathrm{O} i} \omega_{i} \sin \left(\omega_{3}-\omega_{i}\right) t}{\left[R_{\mathrm{O} 3}^{2}+R_{\mathrm{O} i}^{2}-2 R_{\mathrm{O} 3} R_{\mathrm{O} i} \cos \left(\omega_{3}-\omega_{i}\right) t\right]^{3 / 2}}
\end{aligned}
$$

We used $[25,26]$ the maximal positive value $\max (\partial /$ $\partial t) \psi_{3 M}\left(C_{3}\right.$, int) (of the partial derivative $(\partial / \partial t) \psi_{3 M}\left(C_{3}\right.$, int) 
of the gravitational potential $\psi_{3 M}\left(C_{3}\right.$, int $)$ created by Mercury at the mass center $C_{3}$ of the Earth) as a scale of the energy gravitational influence of the planets of the Solar System on the Earth in the considered first approximation of the circular orbits of the planets. To evaluate the relative energy gravitational influence of the inner planet $\tau_{i}$ (Mercury or Venus) at the mass center $C_{3}$ of the Earth, we considered $[25,26]$ the ratio $f\left(i, C_{3}\right)$ of the maximal positive value $\max (\partial / \partial t) \psi_{3 i}\left(C_{3}\right.$, int) (of the partial derivative $(\partial / \partial t) \psi_{3 i}\left(C_{3}\right.$, int) of the gravitational potential $\psi_{3 i}\left(C_{3}\right.$, int $)$ created by the inner planet $\tau_{i}$ at the point $C_{3}$ ) and the maximal positive value $\max \left(\partial \psi_{31}\left(C_{3}\right.\right.$, int $\left.) / \partial t\right)$ (of the partial derivative $\partial \psi_{31}\left(C_{3}\right.$, int)/ $\partial t$ of the gravitational potential $\psi_{31}\left(C_{3}\right.$, int $) \equiv \psi_{3 M}\left(C_{3}\right.$, int) created by Mercury at the mass center $C_{3}$ of the Earth):

$$
f\left(i, C_{3}\right)=\frac{\max \left(\partial \psi_{3 i}\left(C_{3}, \text { int }\right) / \partial t\right)}{\max \left(\partial \psi_{3 M}\left(C_{3}, \text { int }\right) / \partial t\right)}, \quad(i=1,2) .
$$

We obtained $[25,26]$ from expression (108) the obvious value $f\left(1, C_{3}\right)=1$ for Mercury $\tau_{1}$. Using the formula (108) for Venus $(i=2)$, we calculated $[25,26]$ the numerical value $f\left(2, C_{3}\right)=37.69807434$ for the following numerical values [35]: the mass $M_{M}=0.06 M_{3}$ of Mercury, where $M_{3}$ is the mass of the Earth, the mass $M_{V}=M_{2}=0.82 M_{3}$ of Venus, the time period $T_{3}=365.3$ days of the Earth's circulation around the Sun, the time period $T_{M}=88$ days of Mercury's circulation around the Sun, the time period $T_{V}=T_{2}=224.7$ days of the Venusian circulation around the Sun, the average radius $R_{\mathrm{OM}}=R_{\mathrm{O} 1}=57.85 \cdot 10^{6} \mathrm{~km}$ of Mercury's orbit, around the Sun, the average radius $R_{\mathrm{O} 3}=149.6 \cdot 10^{6} \mathrm{~km}$ of the Earth's orbit, and the average radius $R_{\mathrm{OV}}=R_{\mathrm{O} 2}=108.1 \cdot 10^{6} \mathrm{~km}$ of Venusian orbit around the Sun. The power of the maximal energy gravitational Venusian influence (on the unit mass at the mass center $C_{3}$ of the Earth) is $f\left(2, C_{3}\right)=37.69807434$ times larger than the power of the maximal energy gravitational influence of Mercury (on the unit mass at the mass center $C_{3}$ of the Earth).

To evaluate the relative energy gravitational influence on the Earth of the outer planet $\tau_{i}$ at the mass center $C_{3}$ of the Earth, we considered $[25,26]$ the ratio $f\left(i, C_{3}\right)$ (for $i=4,5,6$, $7,8,9)$ of the maximal value $\max (\partial / \partial t) \psi_{3 i}\left(C_{3}\right.$, ext $)$ of the partial derivative of the gravitational potential $\psi_{3 i}\left(C_{3}\right.$, ext $)$ (created by the outer planet $\tau_{i}$ at the mass center $C_{3}$ of the Earth) and the maximal value $\max (\partial / \partial t) \psi_{3 M}\left(C_{3}\right.$, int $)$ of the partial derivative of the gravitational potential $\psi_{3 M}\left(D_{3}\right.$, int) (created by Mercury at the mass center $C_{3}$ of the Earth):

$$
f\left(i, C_{3}\right)=\frac{\max \left(\partial \psi_{3 i}\left(C_{3}, \text { ext }\right) / \partial t\right)}{\max \left(\partial \psi_{3 M}\left(C_{3}, \text { int }\right) / \partial t\right)}, \quad(i=4,5,6,7,8,9) .
$$

Using the formula (109), we calculated $[25,26]$ the following numerical values: $f\left(4, C_{3}\right)=0.67441034$ (for Mars $\tau_{4}, i=$ 4 ), $f\left(5, C_{3}\right)=7.41055774$ (for Jupiter $\left.\tau_{5}, i=5\right), f\left(6, C_{3}\right)=$ 0.24601009 (for Saturn $\tau_{6}, i=6$ ), $f\left(7, C_{3}\right)=0.00319056$ (for Uranus $\tau_{7}, i=7$ ), $f\left(8, C_{3}\right)=0.00077565$ (for Neptune $\tau_{8}, i=8$ ), and $f\left(9, C_{3}\right)=3.4813 \cdot 10^{-8}$ (for Pluto $\tau_{9}, i=9$ ). We used the following additional planetary numerical values [50]: the mass $M_{\mathrm{MARS}}=M_{4}=0.11 M_{3}$ of Mars, the time period $T_{\text {MARS }}=T_{4}=687$ days of Mars' circulation around the Sun, the average radius $R_{\mathrm{OMARS}}=R_{\mathrm{O} 4}=227.7 \cdot 10^{6} \mathrm{~km}$ of Mars' orbit, the mass $M_{J}=M_{5}=318 M_{3}$ of Jupiter, the time period $T_{J}=T_{5}=4332$ days of Jupiter's circulation around the Sun, and the average radius $R_{\mathrm{OJ}}=R_{\mathrm{O} 5}=777.6 \cdot 10^{6} \mathrm{~km}$ of Jupiter's orbit, the time period $T_{\mathrm{SAT}}=T_{6}=10759$ days of Saturn's circulation around the Sun, the mass $M_{\mathrm{SAT}}=M_{6}=$ $95.2 M_{3}$ of Saturn, and the average radius $R_{\mathrm{OSAT}}=R_{\mathrm{O} 6}=$ $1426 \cdot 10^{6} \mathrm{~km}$ of Saturn's orbit, the mass $M_{U}=M_{7}=14.6 M_{3}$ of Uranus, the time period $T_{U}=T_{7}=30685$ days of Uranus' circulation around the Sun, and the average radius $R_{\mathrm{OU}}=R_{\mathrm{O} 7}=2868 \cdot 10^{6} \mathrm{~km}$ of Uranus' orbit, the mass $M_{N}=$ $M_{8}=17.2 M_{3}$ of Neptune, the average radius $R_{\mathrm{ON}}=R_{\mathrm{O} 8}=$ $4497 \cdot 10^{6} \mathrm{~km}$ of Neptune's orbit, and the time period $T_{N}=$ $T_{8}=60189$ days of Neptune's circulation around the Sun, the mass $M_{P}=M_{9}=0.002 M_{3}$ of Pluto, the time period $T_{P}=$ $T_{9}=90465$ days of Pluto's circulation around the Sun, and the average radius $R_{\mathrm{OP}}=R_{\mathrm{O} 9}=5900 \cdot 10^{6} \mathrm{~km}$ of Pluto's orbit.

Taking into account the calculated powers of the maximal energy gravitational influences of the planets on the unit mass of the Earth (at the mass center $C_{3}$ of the Earth), we obtained $[25,26]$ the following numerical sequence of the nondimensional relative maximal powers of the planetary energy gravitational influences on the unit mass of the Earth (at the mass center $C_{3}$ of the Earth): $f\left(2, C_{3}\right)=37.69807434$ (for Venus), $f\left(5, C_{3}\right)=7.41055774$ (for Jupiter), $f\left(1, C_{3}\right)=1$ (for Mercury), $f\left(4, C_{3}\right)=0.67441034$ (for Mars), $f\left(6, C_{3}\right)=$ 0.24601009 (for Saturn), $f\left(7, C_{3}\right)=0.00319056$ (for Uranus), $f\left(8, C_{3}\right)=0.00077565$ (for Neptune), and $f\left(9, C_{3}\right)=3.4813$. $10^{-8}$ (for Pluto).

To evaluate the relative energy gravitational influence of the inner planets $\tau_{i}$ (Mercury and Venus) and the outer planets $\tau_{i}$ (Mars, Jupiter, Saturn, Uranus, Neptune, and Pluto) at the surface point $D_{3}$ (which is the intersection of the direct line (connecting the mass center $O$ of the Sun and the mass center $C_{3}$ of the Earth) with the surface of the Earth), we obtained [12] the gravitational potential $\psi_{3 i}\left(D_{3}\right.$, int $)$ :

$$
\begin{aligned}
& \psi_{3 i}\left(D_{3}, \text { int }\right) \\
& =-\frac{\gamma M_{i}}{\sqrt{\left(R_{\mathrm{O} 3}-R_{3}\right)^{2}+R_{\mathrm{O} i}^{2}-2\left(R_{\mathrm{O} 3}-R_{3}\right) R_{\mathrm{O} i} \cos \left(\omega_{i} t-\varphi_{3}\right)}}
\end{aligned}
$$

created by the inner planet $\tau_{i}$ in the surface point $D_{3}$ of the Earth. We derived the expression for the partial derivative $(\partial / \partial t) \psi_{3 i}\left(D_{3}\right.$, int $)$ of the gravitational potential (110):

$$
\begin{aligned}
& \frac{\partial}{\partial t} \psi_{3 i}\left(D_{3}, \text { int }\right) \\
= & \frac{\gamma M_{i}\left(R_{\mathrm{O} 3}-R_{3}\right) R_{\mathrm{O} i} \omega_{i} \sin \left(\omega_{i}-\omega_{3}\right) t}{\left[\left(R_{\mathrm{O} 3}-R_{3}\right)^{2}+R_{\mathrm{O} i}^{2}-2\left(R_{\mathrm{O} 3}-R_{3}\right) R_{\mathrm{O} i} \cos \left(\omega_{i}-\omega_{3}\right) t\right]^{3 / 2}} .
\end{aligned}
$$


The gravitational potential created by the outer planet $\tau_{i}$ at the surface point $D_{3}$ (for configuration shown on Figure 8 ) is given by the following expression [12]:

$$
\begin{aligned}
& \psi_{3 i}\left(D_{3}, \text { ext }\right) \\
& =-\frac{\gamma M_{i}}{d_{3 i}\left(D_{3}\right)} \\
& =-\frac{\gamma M_{i}}{\sqrt{\left(R_{\mathrm{O} 3}-R_{3}\right)^{2}+R_{\mathrm{O} i}^{2}-2\left(R_{\mathrm{O} 3}-R_{3}\right) R_{\mathrm{O} i} \cos \left(\varphi_{3}-\omega_{i} t\right)}} .
\end{aligned}
$$

We derived [12] the expression of the partial derivative $(\partial / \partial t) \psi_{3 i}\left(D_{3}\right.$, ext) of the gravitational potential (112):

$$
\begin{aligned}
\frac{\partial}{\partial t} \psi_{3 i} & \left(D_{3}, \text { ext }\right) \\
= & -\left(\gamma M_{i}\left(R_{\mathrm{O} 3}-R_{3}\right) R_{\mathrm{O} i} \omega_{i} \sin \left(\omega_{3}-\omega_{i}\right) t\right) \\
& \times\left(\left[\left(R_{\mathrm{O} 3}-R_{3}\right)^{2}+R_{\mathrm{O} i}^{2}\right.\right. \\
& \left.\left.-2\left(R_{\mathrm{O} 3}-R_{3}\right) R_{\mathrm{O} i} \cos \left(\omega_{3}-\omega_{i}\right) t\right]^{3 / 2}\right)^{-1},
\end{aligned}
$$

which is reduced to the relation (111) owing to the equalities $\sin \left(\omega_{i}-\omega_{3}\right) t=-\sin \left(\omega_{3}-\omega_{i}\right) t$ and $\cos \left(\omega_{i}-\omega_{3}\right) t=\cos \left(\omega_{3}-\omega_{i}\right) t$. However, we take into account that the expression (113) is given for the outer planet $\tau_{i}$, but the expression (111) is given for the inner planet $\tau_{i}$.

We used $[12,25,26]$ the maximal positive value $\max (\partial /$ $\partial t) \psi_{3 M}\left(D_{3}\right.$, int) (of the partial derivative $(\partial / \partial t) \psi_{3 M}\left(D_{3}\right.$, int) of the gravitational potential $\psi_{3 M}\left(D_{3}\right.$, int $)$ created by Mercury at the surface point $D_{3}$ of the Earth) as a scale of the energy gravitational influence of the planets of the Solar System on the Earth in the considered first approximation of the circular orbits of the planets.

To evaluate the relative energy gravitational influence of the inner planet $\tau_{i}(i=1,2)$ at the surface point $D_{3}$, we considered $[12,25,26]$ the ratio $f\left(i, D_{3}\right)$ of the maximal positive value $\max (\partial / \partial t) \psi_{3 i}\left(D_{3}\right.$, int) (of the partial derivative $(\partial / \partial t) \psi_{3 i}\left(D_{3}\right.$,int $)$ of the gravitational potential $\psi_{3 i}\left(D_{3}\right.$, int $)$ created by the inner planet $\tau_{i}$ at the point $\left.D_{3}\right)$ and the maximal positive value $\max (\partial / \partial t) \psi_{3 M}\left(D_{3}\right.$, int) (of the partial derivative $(\partial / \partial t) \psi_{3 M}\left(D_{3}\right.$, int $)$ of the gravitational potential $\psi_{31}\left(D_{3}\right.$, int $) \equiv \psi_{3 M}\left(D_{3}\right.$, int $)$ created by Mercury at the surface point $D_{3}$ of the Earth):

$$
f\left(i, D_{3}\right)=\frac{\max \left(\partial \psi_{3 i}\left(D_{3}, \text { int }\right) / \partial t\right)}{\max \left(\partial \psi_{3 M}\left(D_{3}, \text { int }\right) / \partial t\right)}, \quad(i=1,2) .
$$

To evaluate the relative energy gravitational influence of the outer planet $\tau_{i}(i=4,5,6,7,8,9)$ on the surface point $D_{3}$ of the Earth, we considered $[12,25,26]$ the ratio $f\left(i, D_{3}\right)$ (for $i=4,5,6,7,8,9$ ) of the maximal positive value $\max (\partial / \partial t) \psi_{3 i}\left(D_{3}\right.$, ext) (of the partial derivative $(\partial / \partial t) \psi_{3 i}\left(D_{3}\right.$, ext) of the gravitational potential $\psi_{3 i}\left(D_{3}\right.$, ext) created by the outer planet $\tau_{i}$ at the surface point $\left.D_{3}\right)$ and the maximal positive value $\max (\partial / \partial t) \psi_{3 M}\left(D_{3}\right.$, int) (of the partial derivative $(\partial / \partial t) \psi_{3 M}\left(D_{3}\right.$, int $)$ of the gravitational potential $\psi_{3 M}\left(D_{3}\right.$, int $)$ created by Mercury at surface point $D_{3}$ ):

$$
f\left(i, D_{3}\right)=\frac{\max \left(\partial \psi_{3 i}\left(D_{3}, \text { ext }\right) / \partial t\right)}{\max \left(\partial \psi_{3 M}\left(D_{3}, \text { int }\right) / \partial t\right)}, \quad(i=4,5,6,7,8,9) .
$$

Using the formulae (114) and (115), we calculated [25, 26] (for the average radius $R_{3}=6371 \mathrm{~km}$ of the Earth and the planetary numerical values [50]) the following numerical values (corrected slightly the previous numerical values of $\left.f\left(i, D_{3}\right)[9]\right): f\left(2, D_{3}\right)=37.70428085$ (for Venus), $f\left(5, D_{3}\right)=$ 7.40926122 (for Jupiter), $f\left(1, D_{3}\right)=1$ (for Mercury), $f\left(4, D_{3}\right)=0.67420160$ (for Mars), $f\left(6, D_{3}\right)=0.24596865$ (for Saturn), $f\left(7, D_{3}\right)=0.00319004$ (for Uranus), $f\left(8, D_{3}\right)=$ 0.00077552 (for Neptune), and $f\left(9, D_{3}\right)=3.4807 \cdot 10^{-8}$ (for Pluto).

Taking into account the calculated powers of the maximal energy gravitational influences of the planets on the unit mass of the Earth (at the mass center $C_{3}$ of the Earth and at the surface point $D_{3}$ of the Earth), we obtained $[12,25,26]$ the following order of signification of the planets of the Solar System (Venus, Jupiter, Mercury, Mars, Saturn, Uranus, Neptune, and Pluto) with respect to the planetary power of the maximal energy gravitational influences on the unit mass of the Earth.

To evaluate the relative energy gravitational influence of the inner planet $\tau_{i}$ at the surface point $D_{3}$ and at the mass center $C_{3}$ of the Earth, we considered $[25,26]$ the ratio $q_{i}\left(D_{3}, C_{3}\right)$ :

$$
q_{i}\left(D_{3}, C_{3}\right)=\frac{\max \left(\partial \psi_{3 i}\left(D_{3}, \text { int }\right) / \partial t\right)}{\max \left(\partial \psi_{3 i}\left(C_{3}, \text { int }\right) / \partial t\right)}, \quad(i=1,2) .
$$

To evaluate the relative energy gravitational influence of the outer planet $\tau_{i}$ at the surface point $D_{3}$ and at the mass center $C_{3}$ of the Earth, we considered $[25,26]$ the ratio $q_{i}\left(D_{3}, C_{3}\right)$ :

$$
q_{i}\left(D_{3}, C_{3}\right)=\frac{\max \left(\partial \psi_{3 i}\left(D_{3}, \text { ext }\right) / \partial t\right)}{\max \left(\partial \psi_{3 i}\left(C_{3}, \text { ext }\right) / \partial t\right)}, \quad(i=4,5,6,7,8,9) .
$$

Using the formula (116), we calculated $[25,26]$ the following numerical values: $q_{1}\left(D_{3}, C_{3}\right)=1.000123023$ (for Mercury) and $q_{2}\left(D_{3}, C_{3}\right)=1.000287771$ (for Venus). Using the formula (117), we calculated $[25,26]$ the following numerical values: $q_{4}\left(D_{3}, C_{3}\right)=0.999813318$ (for Mars), $q_{5}\left(D_{3}, C_{3}\right)=$ 0.999948084 (for Jupiter), $q_{6}\left(D_{3}, C_{3}\right)=0.999954640$ (for Saturn), $q_{7}\left(D_{3}, C_{3}\right)=0.999956727$ (for Uranus), $q_{8}\left(D_{3}, C_{3}\right)=$ 0.999957084 (for Neptune), and $q_{9}\left(D_{3}, C_{3}\right)=0.999957263$ (for Pluto).

The revealed $[25,26]$ small difference of the maximal energy gravitational influence of each planet at the surface point $D_{3}$ and at the mass center $C_{3}$ of the Earth must lead to the small difference of the combined maximal energy gravitational influences of the planets of the Solar System at the points $C_{3}$ and $D_{3}$ of the Earth. It was recognized $[25,26]$ that the small difference of the combined planetary maximal energy gravitational influences at the surface point $D_{3}$ and at the mass center $C_{3}$ of the Earth can contribute to the following related geophysical phenomena: the small oscillatory 
motion of the rigid kernel of the Earth relative to the fluid kernel of the Earth; the small oscillation of the Earth's pole (i.e., the Chandler's wobble of the Earth's pole); the small oscillations of the boundary of the Pacific Ocean (i.e., the seismic zone of the Pacific Ring); the oscillations, rotations, and deformations of the geoblocks weakly coupled with the surrounding plastic layers in all seismic zones of the Earth and the formation of fractures related to the strong earthquakes and the planetary cataclysms.

4.1.3. The Evaluation of the Relative Maximal Planetary Integral Energy Gravitational Influences on the Earth in the Approximation of the Circular Orbits of the Planets of the Solar System. We assume that $\varphi_{03}$ and $\varphi_{0 i}$ are the initial phases of the Earth $\tau_{3}$ and the planet $\tau_{i}$, respectively. Consequently, the positions of the center of the Earth $\tau_{3}$ and the center of the planet $\tau_{i}$ (inner or outer) for the time moment $t$ are given (instead of the relation (100)) by the following expressions:

$$
\begin{gathered}
\varphi_{i}(t)=\omega_{i} t=\frac{2 \pi}{T_{i}} t+\varphi_{0 i}, \\
\varphi_{3}(t)=\omega_{3} t=\frac{2 \pi}{T_{3}} t+\varphi_{03} .
\end{gathered}
$$

Taking into account the initial phases $\varphi_{03}$ and $\varphi_{0 i}$, the expressions (104), (107), (111), and (113) can be generalized as follows:

$$
\begin{aligned}
& \frac{\partial}{\partial t} \psi_{3 i}\left(C_{3}, \text { int }\right) \\
& =\frac{\partial}{\partial t} \psi_{3 i}\left(C_{3}, \text { ext }\right)=\frac{\partial}{\partial t} \psi_{3 i}\left(C_{3}\right) \\
& =\frac{\gamma M_{i} R_{\mathrm{O} 3} R_{\mathrm{O} i} \omega_{i} \sin \left\{\left(\omega_{i}-\omega_{3}\right) t+\varphi_{0 i}-\varphi_{03}\right\}}{\left[R_{\mathrm{O} 3}^{2}+R_{\mathrm{O} i}^{2}-2 R_{\mathrm{O} 3} R_{\mathrm{O} i} \cos \left\{\left(\omega_{i}-\omega_{3}\right) t+\varphi_{0 i}-\varphi_{03}\right\}\right]^{3 / 2}}
\end{aligned}
$$

$$
\begin{aligned}
& \frac{\partial}{\partial t} \psi_{3 i}\left(D_{3}, \text { int }\right) \\
&= \frac{\partial}{\partial t} \psi_{3 i}\left(D_{3}, \text { ext }\right)=\frac{\partial}{\partial t} \psi_{3 i}\left(D_{3}\right) \\
&=\left(\gamma M_{i}\left(R_{\mathrm{O} 3}-R_{3}\right) R_{\mathrm{O} i} \omega_{i}\right. \\
&\left.\quad \times \sin \left\{\left(\omega_{i}-\omega_{3}\right) t+\varphi_{0 i}-\varphi_{03}\right\}\right) \\
& \quad \times\left(\left[\left(R_{\mathrm{O} 3}-R_{3}\right)^{2}+R_{\mathrm{O} i}^{2}-2\left(R_{\mathrm{O} 3}-R_{3}\right) R_{\mathrm{O} i}\right.\right. \\
&\left.\left.\quad \times \cos \left\{\left(\omega_{i}-\omega_{3}\right) t+\varphi_{0 i}-\varphi_{03}\right\}\right]^{3 / 2}\right)^{-1} .
\end{aligned}
$$

We obtained [12] the integral energy gravitational influence $\Delta_{g} E_{3}\left(\tau_{i}, \varphi_{0 i}, \varphi_{03}, t, t_{0}\right)$ on the Earth $\tau_{3}$ owing to the nonstationary instantaneous energy gravitational influence of the planet $\tau_{i}$ (inner or outer) during the time interval $\left(t_{0}, t\right)$ :

$$
\begin{aligned}
& \Delta_{g} E_{3}\left(\tau_{i}, \varphi_{0 i}, \varphi_{03}, t, t_{0}\right) \\
& \quad=\int_{t_{0}}^{t}\left(\iiint_{\tau_{3}}\left(\frac{\partial \psi_{3 i}\left(C_{3}\right)}{\partial t^{\prime}}\right) \rho d V\right) d t^{\prime} \\
& \quad \approx M_{3} \int_{t_{0}}^{t}\left(\frac{\partial \psi_{3 i}\left(C_{3}\right)}{\partial t^{\prime}}\right) d t^{\prime},
\end{aligned}
$$

where $M_{3}$ is the mass of the Earth. Substituting (119) into (121), we obtained [12]

$$
\begin{aligned}
& \Delta_{g} E_{3}\left(\tau_{i}, \varphi_{0 i}, \varphi_{03}, t, t_{0}\right) \\
&=M_{3} \int_{t_{0}}^{t}\left(\gamma M_{i} R_{\mathrm{O} 3} R_{\mathrm{O} i} \omega_{i} \sin \left\{\left(\omega_{i}-\omega_{3}\right) t^{\prime}+\varphi_{0 i}-\varphi_{03}\right\}\right) \\
& \times\left(\left[\left(R_{\mathrm{O} 3}\right)^{2}+R_{\mathrm{O} i}^{2}-2 R_{\mathrm{O} 3} R_{\mathrm{O} i}\right.\right. \\
&\left.\left.\quad \times \cos \left\{\left(\omega_{i}-\omega_{3}\right) t^{\prime}+\varphi_{0 i}-\varphi_{03}\right\}\right]^{3 / 2}\right)^{-1} d t^{\prime} .
\end{aligned}
$$

The result of integration of the expression (122) is given by the relation [12]:

$$
\begin{aligned}
\Delta_{g} E_{3}\left(\tau_{i}, \varphi_{0 i}, \varphi_{03}, t, t_{0}\right) & =\frac{2 \alpha_{i}}{\left(\omega_{i}-\omega_{3}\right) \chi_{i}}\left[\frac{1}{\left[\beta_{i}-\chi_{i} \cos \left\{\left(\omega_{i}-\omega_{3}\right) t_{0}+\varphi_{0 i}-\varphi_{03}\right\}\right]^{1 / 2}}\right. \\
& \left.-\frac{1}{\left[\beta_{i}-\chi_{i} \cos \left\{\left(\omega_{i}-\omega_{3}\right) t+\varphi_{0 i}-\varphi_{03}\right\}\right]^{1 / 2}}\right],
\end{aligned}
$$

characterized by the following coefficients:

$$
\begin{gathered}
\alpha_{i}=\gamma M_{3} M_{i} R_{\mathrm{O} 3} R_{\mathrm{O} i} \omega_{i}, \\
\beta_{i}=\left(R_{\mathrm{O} 3}\right)^{2}+\left(R_{\mathrm{O} i}\right)^{2}, \\
\chi_{i}=2 R_{\mathrm{O} 3} R_{\mathrm{O} i} .
\end{gathered}
$$

Using the initial phases $\varphi_{03}=0$ and $\varphi_{0 i}=0$ for the initial time moment $t_{0}=0$, the expression (123) gives the more simple relation [12]:

$$
\begin{aligned}
\Delta_{g} E_{3}\left(\tau_{i}, 0,0, t, 0\right) & \\
=\frac{2 \alpha_{i}}{\left(\omega_{i}-\omega_{3}\right) \chi_{i}} & {\left[\frac{1}{\left[\beta_{i}-\chi_{i}\right]^{1 / 2}}\right.} \\
& \left.-\frac{1}{\left[\beta_{i}-\chi_{i} \cos \left\{\left(\omega_{i}-\omega_{3}\right) t\right\}\right]^{1 / 2}}\right]
\end{aligned}
$$

used for calculation of the maximal integral energy gravitational influence of the planet $\tau_{i}$ (inner or outer) on the Earth 
$\tau_{3}$. Consider the expression (125) by taking into account that the mass center $C_{i}$ of the inner planet $\tau_{i}$, the mass center $C_{3}$ of the Earth $\tau_{3}$, and the mass center $O$ of the Sun are located on the axis $X$ for the initial time moment $t_{0}=0$ as it is shown on Figure 6. Considering the time duration

$$
t_{1}^{*}(i, 3)=\frac{1}{2} \frac{T_{i} T_{3}}{\left(T_{3}-T_{i}\right)}, \quad(i=1,2)
$$

we obtain that the mass center $C_{i}$ of the inner planet $\tau_{i}$, the mass center $C_{3}$ of the Earth $\tau_{3}$, and the mass center $O$ of the Sun will be localized again on the same straight line and the distance between the mass centers of the inner planet $\tau_{i}$, and the Earth $\tau_{3}$ will be maximal. We have from relation (125) the positive integral energy gravitational influence on the Earth's continuum during the time $t_{1}^{*}(i, 3)$ :

$$
\begin{aligned}
\Delta_{g} E_{3}\left(\tau_{i}, 0,0, t_{1}^{*}(i, 3), 0\right) \\
\quad=\frac{2 \alpha_{i}}{\left(\omega_{i}-\omega_{3}\right) \chi_{i}}\left[\frac{1}{\left[R_{\mathrm{O} 3}-R_{\mathrm{O} i}\right]}-\frac{1}{\left[R_{\mathrm{O} 3}+R_{\mathrm{O} i}\right]}\right]>0 .
\end{aligned}
$$

We obtained [12] from relation (125) that the integral energy gravitational influence on the Earth of the inner planet $\tau_{i}$ is equal to the zero

$$
\Delta_{g} E_{3}\left(\tau_{i}, 0,0, t_{2}^{*}(i, 3), 0\right)=0
$$

during the time duration

$$
t_{2}^{*}(i, 3)=\frac{T_{i} T_{3}}{\left(T_{3}-T_{i}\right)}, \quad(i=1,2)
$$

when the distance between the mass centers of the inner planet $\tau_{i}$ and the Earth $\tau_{3}$ will be minimal.

We tested [12] the relation (125) for the outer planet $\tau_{i}$ and the Earth $\tau_{3}$ assuming that the mass centers of the outer planet $\tau_{i}$ and the Earth $\tau_{3}$ and the mass center $O$ of the Sun are located on the axis $X$ for the initial time moment $t_{0}=0$ as it is shown on Figure 6. Considering the time duration

$$
t_{1}^{*}(3, i)=\frac{1}{2} \frac{T_{i} T_{3}}{\left(T_{i}-T_{3}\right)}, \quad(i=4,5,6,7,8,9)
$$

we have that the mass center of the outer planet $\tau_{i}$, the mass center $\mathrm{C}_{3}$ of the Earth $\tau_{3}$, and the mass center $\mathrm{O}$ of the Sun will again be located on the same straight line, and the distance between the mass centers of the outer planet $\tau_{i}$ and the Earth $\tau_{3}$ will be maximal. We obtained [12] from relation (125) the negative integral energy gravitational influence on the Earth of outer planet $\tau_{i}$ during the time $t_{1}^{*}(3, i)$ :

$$
\begin{aligned}
\Delta_{g} & E_{3}\left(\tau_{i}, 0,0, t_{1}^{*}(3, i), 0\right) \\
& =\frac{2 \alpha_{i}}{\left(\omega_{i}-\omega_{3}\right) \chi_{i}}\left[\frac{1}{\left[R_{\mathrm{O} i}-R_{\mathrm{O} 3}\right]}-\frac{1}{\left[R_{\mathrm{O} i}+R_{\mathrm{O} 3}\right]}\right]<0 .
\end{aligned}
$$

Considering the time duration

$$
t_{2}^{*}(3, i)=\frac{T_{i} T_{3}}{\left(T_{i}-T_{3}\right)},
$$

we obtained [12] from relation (125) the zero integral energy gravitational influence on the Earth from the outer planet $\tau_{i}$ during the time $t_{2}^{*}(3, i)$ :

$$
\Delta_{g} E_{3}\left(\tau_{i}, 0,0, t_{2}^{*}(3, i), 0\right)=0 .
$$

Using the expression (124), we established [12] that the expressions (127) and (131) give the following extreme values:

$$
\begin{aligned}
& \max _{t} \Delta_{g} E_{3}\left(\tau_{i}, 0,0, t, 0\right) \\
& =\Delta_{g} E_{3}\left(\tau_{i}, 0,0, t_{1}^{*}(i, 3), 0\right) \\
& =2 \gamma M_{3} M_{i} \frac{R_{\mathrm{O} i} T_{3}}{\left(R_{\mathrm{O} 3}^{2}-R_{\mathrm{O} i}^{2}\right)\left(T_{3}-T_{i}\right)}>0, \\
& \min _{t} \Delta_{g} E_{3}\left(\tau_{i}, 0,0, t, 0\right) \quad(i=1,2), \\
& =\Delta_{g} E_{3}\left(\tau_{i}, 0,0, t_{1}^{*}(3, i), 0\right) \\
& =2 \gamma M_{3} M_{i} \frac{R_{\mathrm{O} 3} T_{3}}{\left(R_{\mathrm{O} i}^{2}-R_{\mathrm{O} 3}^{2}\right)\left(T_{3}-T_{i}\right)}<0, \\
& (i=4,5,6,7,8,9)
\end{aligned}
$$

of the integral energy gravitational influences (respectively, the positive maximal integral energy gravitational influence from the inner planet $\tau_{i}$ and the negative minimal integral energy gravitational influence from the outer planet $\tau_{i}$ ) for the given initial phases $\varphi_{03}=0$ and $\varphi_{0 i}=0$ (for the initial time moment $t_{0}=0$ ) corresponding to the initial configurations shown on Figures 5 and 6.

Using relation (134), we obtained [12] for Mercury $(i=1)$ and for Venus $(i=2)$ the following expressions of the maximal positive integral energy gravitational influences on the Earth:

$$
\begin{aligned}
& \max _{t} \Delta_{g} E_{3}\left(\tau_{1}, 0,0, t, 0\right) \\
&=\Delta_{g} E_{3}\left(\tau_{1}, 0,0, t_{1}^{*}(1,3), 0\right) \\
&=2 \gamma M_{3} M_{1} \frac{R_{\mathrm{O} 1} T_{3}}{\left(R_{\mathrm{O} 3}^{2}-R_{\mathrm{O} 1}^{2}\right)\left(T_{3}-T_{1}\right)}>0, \quad i=1, \\
& \max _{t} \Delta_{g} E_{3}\left(\tau_{2}, 0,0, t, 0\right) \\
&=\Delta_{g} E_{3}\left(\tau_{2}, 0,0, t_{1}^{*}(2,3), 0\right) \\
& \quad=2 \gamma M_{3} M_{2} \frac{R_{\mathrm{O} 2} T_{3}}{\left(R_{\mathrm{O} 3}^{2}-R_{\mathrm{O} 2}^{2}\right)\left(T_{3}-T_{2}\right)}>0, \quad i=2 .
\end{aligned}
$$

Considering the mass $m_{\tau}$ of the macroscopic continuum region near the surface point $D_{3}$ (instead of the mass $M_{3}$ of the Earth) in relations (136) and (137), we obtained [12] the following expressions for the positive integral energy gravitational influences of Mercury $(i=1)$ and Venus $(i=2)$ on 
the macroscopic continuum region of the mass $m_{\tau}$ near the surface point $D_{3}$ of the Earth:

$$
\begin{aligned}
& \max _{t} \Delta_{g} E_{3}\left(\tau_{1}, D_{3}, m_{\tau}, 0,0, t, 0\right) \\
& \quad=\Delta_{g} E_{3}\left(\tau_{1}, D_{3}, m_{\tau}, 0,0, t_{1}^{*}(1,3), 0\right) \\
& \quad=2 \gamma m_{\tau} M_{1} \frac{R_{\mathrm{O} 1} T_{3}}{\left(R_{\mathrm{O} 3}^{2}-R_{\mathrm{O} 1}^{2}\right)\left(T_{3}-T_{1}\right)}>0, \quad i=1, \\
& \max _{t} \Delta_{g} E_{3}\left(\tau_{2}, D_{3}, m_{\tau}, 0,0, t, 0\right) \\
& \quad=\Delta_{g} E_{3}\left(\tau_{2}, D_{3}, m_{\tau}, 0,0, t_{1}^{*}(2,3), 0\right) \\
& \quad=2 \gamma m_{\tau} M_{2} \frac{R_{\mathrm{O} 2} T_{3}}{\left(R_{\mathrm{O} 3}^{2}-R_{\mathrm{O} 2}^{2}\right)\left(T_{3}-T_{2}\right)}>0, \quad i=2 .
\end{aligned}
$$

We shall use the expression (136) as a measuring unit for evaluations of the maximal absolute values of the integral energy gravitational influences on the Earth of the planets of the Solar System and the Moon.

Considering the ratio of the extreme value $\max _{t} \Delta_{g} E_{3}\left(\tau_{i}\right.$, $0,0, t, 0)$ (given by expression (134)) and the maximal positive integral energy gravitational influence $\max _{t} \Delta_{g} E_{3}\left(\tau_{1}, 0,0, t\right.$, 0 ) (given by the expression (136)) of Mercury on the Earth, we obtained [12] the relative values $s(i)$ of the maximal integral energy gravitational influences on the Earth of the inner planets:

$$
\begin{aligned}
s(i) & =\frac{\max _{t} \Delta_{g} E_{3}\left(\tau_{i}, 0,0, t, 0\right)}{\max _{t} \Delta_{g} E_{3}\left(\tau_{1}, 0,0, t, 0\right)} \\
& =\frac{M_{i}}{M_{1}} \frac{R_{\mathrm{O} i}\left(R_{\mathrm{O} 3}^{2}-R_{\mathrm{O} 1}^{2}\right)\left(T_{3}-T_{1}\right)}{R_{\mathrm{O} 1}\left(R_{\mathrm{O} 3}^{2}-R_{\mathrm{O} i}^{2}\right)\left(T_{3}-T_{i}\right)}, \quad i=1,2 .
\end{aligned}
$$

We have the obvious value $s(1)=1$ for Mercury $(i=1)$. We calculated [12] the value $s(2)=89.6409$ for Venus $(i=2)$ based on the planetary numerical values [50] of the average radii of the orbits, the time periods of circulations around the Sun, and the masses of the Earth, Venus, and Mercury.

Since the values given by the expression (135) are negative for the outer planets, we used the absolute (positive) value

$$
\begin{aligned}
& \left|\min _{t} \Delta_{g} E_{3}\left(\tau_{i}, 0,0, t, 0\right)\right| \\
& =\left|\Delta_{g} E_{3}\left(\tau_{i}, 0,0, t_{1}^{*}(3, i), 0\right)\right| \\
& =2 \gamma M_{3} M_{i} \frac{R_{\mathrm{O} 3} T_{3}}{\left(R_{\mathrm{O} i}^{2}-R_{\mathrm{O} 3}^{2}\right)\left(T_{i}-T_{3}\right)}>0, \\
& i=4,5,6,7,8,9 .
\end{aligned}
$$

Using the expressions (141) and (136), we obtained [12] the relative values $s(i)$ of the maximal integral energy gravitational influences on the Earth of the outer planets of the Solar System $(i=4,5,6,7,8,9)$ :

$$
\begin{array}{r}
s(i)=\frac{\left|\min _{t} \Delta_{g} E_{3}\left(\tau_{i}, 0,0, t, 0\right)\right|}{\max _{t} \Delta_{g} E_{3}\left(\tau_{1}, 0,0, t, 0\right)} \\
=\frac{M_{i}}{M_{1}} \frac{R_{\mathrm{O} 3}\left(R_{\mathrm{O} 3}^{2}-R_{\mathrm{O} 1}^{2}\right)\left(T_{3}-T_{1}\right)}{R_{\mathrm{O} 1}\left(R_{\mathrm{O} i}^{2}-R_{\mathrm{O} 3}^{2}\right)\left(T_{i}-T_{3}\right)}, \\
i=4,5,6,7,8,9 .
\end{array}
$$

Based on the planetary numerical values [50], we calculated the following numerical values [9]: $s(4)=2.6396$ for Mars $(i=4), s(5)=31.319$ for Jupiter $(i=5), s(6)=1.036$ for Saturn $(i=6), s(7)=0.0133$ for Uranus $(i=7), s(8)=$ 0.003229 for Neptune $(i=8)$, and $s(9)=1.4495 \cdot 10^{-7}$ for Pluto $(i=9)$.

Taking into account the calculated relative values $s(i)$, we obtained [9] the following order of signification of the planets of the Solar System: Venus $(s(2)=89.6409)$, Jupiter $(s(5)=31.319)$, Mars $(s(4)=2.6396)$, Saturn $(s(6)=1.036)$, Mercury $(s(1)=1)$, Uranus $(s(7)=0.0133)$, Neptune $(s(8)=$ $0.003229)$, and Pluto $\left(s(9)=1.4495 \cdot 10^{-7}\right)$ with respect to the established significance of the planetary maximal integral energy gravitational influences on the Earth.

We established [12] that Venus and Jupiter induce the main maximal integral energy gravitational influences on the Earth. Mars, Saturn, and Mercury induce the combined maximal integral energy gravitational influence on the Earth, which is one order of the magnitude smaller than the maximal integral energy gravitational influence of Jupiter. The maximal integral energy gravitational influences on the Earth of Uranus, Neptune, and Pluto are two, three, and seven orders of the magnitude, respectively, smaller than the maximal integral energy gravitational influence of Mercury.

\subsection{The Energy Gravitational Influences on the Earth of the Moon}

4.2.1. The Evaluation of the Relative Maximal Instantaneous Energy Gravitational Influence of the Moon on the Earth in the Second Approximation of the Elliptical Orbits of the Earth and the Moon around the Combined Mass Center $C_{3, M O O N}$ of the Earth and the Moon. We shall consider the movement of the Earth $\tau_{3}$ and the Moon along the elliptical orbits around the combined mass center $C_{3, \text { MOON }}$ of the Earth and the Moon. We have the following relations (see Figure 9):

$$
\begin{aligned}
& r_{\mathrm{MOON}}\left(\varphi_{\mathrm{MOON}}(t)\right)=\frac{p_{\mathrm{MOON}}}{\left(1+e_{\mathrm{MOON}} \cos \varphi_{\mathrm{MOON}}(t)\right)}, \\
& r_{E}\left(\varphi_{E}(t)\right)=r_{E}\left(\varphi_{\mathrm{MOON}}(t)+\pi\right) \\
&=\frac{p_{E}}{\left(1-e_{E} \cos \left(\varphi_{\mathrm{MOON}}(t)+\pi\right)\right)} \\
&=\frac{p_{E}}{\left(1+e_{E} \cos \varphi_{\mathrm{MOON}}(t)\right)}
\end{aligned}
$$




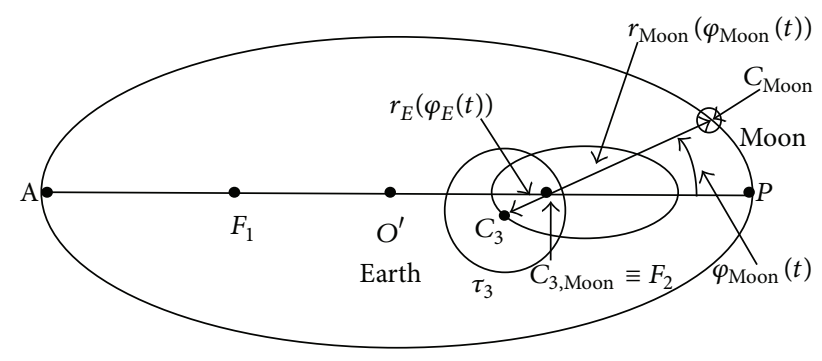

FIGURE 9: The geometric sketch of circulation of the mass center $C_{3}$ of the Earth $\tau_{3}$ and the mass center $C_{\mathrm{MOON}}$ of the Moon around the combined mass center $C_{3, \text { MOON }}$ of the Earth-Moon system.

for the distance $r_{\text {MOON }}\left(\varphi_{\text {MOON }}(t)\right)$ between the combined mass center $C_{3, \mathrm{MOON}}$ and the mass center $C_{\mathrm{MOON}}$ of the Moon and for the distance $r_{E}\left(\varphi_{E}(t)\right)$ between the combined mass center $C_{3, \text { MOON }}$ and the mass center $C_{3}$ of the Earth $\tau_{3}$. We have the focal parameter $p_{\mathrm{MOON}}$ and the eccentricity $e_{\mathrm{MOON}}$ of the elliptical orbit of the Moon. $p_{E}$ and $e_{E}\left(e_{E}=\right.$ $\left.e_{\mathrm{MOON}}\right)$ are the focal parameter and the eccentricity, respectively, of the elliptical Earth's orbit around the combined mass center $C_{3, \mathrm{MOON}}$. We have the expression for the distance $d_{3 M}\left(E_{M}\right)$

$$
\begin{aligned}
d_{3 M}\left(E_{M}\right)= & \frac{p_{\mathrm{MOON}}}{\left(1+e_{\mathrm{MOON}} \cos \varphi_{\mathrm{MOON}}(t)\right)} \\
& +\frac{p_{E}}{\left(1+e_{E} \cos \varphi_{\mathrm{MOON}}(t)\right)}-R_{3}
\end{aligned}
$$

between the mass center $C_{\mathrm{MOON}}$ of the Moon and the point $E_{M}$, which is the intersection of the direct line (connecting the mass center $C_{\mathrm{MOON}}$ of the Moon and the mass center $C_{3}$ of the Earth $\tau_{3}$ ) with the surface of the Earth $\tau_{3}$. We have the expression for the gravitational potential $\psi_{3, \mathrm{MOON}}\left(E_{M}\right)$ created by the Moon at the point $E_{M}$ of the Earth $\tau_{3}$ :

$$
\psi_{3, \mathrm{MOON}}\left(E_{M}\right)=-\gamma \frac{M_{\mathrm{MOON}}}{d_{3 M}\left(E_{M}\right)} .
$$

We obtained $[25,26]$ from the relation $(146)$ the expression for the partial derivative $(\partial / \partial t) \psi_{3, \mathrm{MOON}}\left(E_{M}\right)$ of the gravitational potential $\psi_{3, \text { MOON }}\left(E_{M}\right)$ created by the Moon at the point $E_{M}$ of the Earth $\tau_{3}$ :

$$
\begin{aligned}
\frac{\partial}{\partial t} \psi_{3, \mathrm{MOON}}\left(E_{M}\right) & \\
= & \frac{\gamma M_{\mathrm{MOON}} e_{\mathrm{MOON}} p_{\mathrm{MOON}} \sin \left(\varphi_{\mathrm{MOON}}(t)\right)}{\left(p_{\mathrm{MOON}}+p_{E}-R_{3}\left(1-e_{\mathrm{MOON}} \cos \varphi_{\mathrm{MOON}}(t)\right)\right)^{2}} \\
& \times \frac{d \varphi_{\mathrm{MOON}}(t)}{d t} .
\end{aligned}
$$

We have the expression for the distance $d_{3 M}\left(C_{3}\right)$

$$
\begin{aligned}
d_{3 M}\left(C_{3}\right)= & \frac{p_{\mathrm{MOON}}}{\left(1+e_{\mathrm{MOON}} \cos \varphi_{\mathrm{MOON}}(t)\right)} \\
& +\frac{p_{E}}{\left(1+e_{E} \cos \varphi_{\mathrm{MOON}}(\mathrm{t})\right)}
\end{aligned}
$$

between the mass center $C_{\mathrm{MOON}}$ of the Moon and the mass center $C_{3}$ of the Earth $\tau_{3}$. We have the expression for the gravitational potential $\psi_{3, \mathrm{MOON}}\left(C_{3}\right)$ created by the Moon at the mass center $C_{3}$ of the Earth $\tau_{3}$ :

$$
\psi_{3, \mathrm{MOON}}\left(C_{3}\right)=-\gamma \frac{M_{\mathrm{MOON}}}{d_{3 M}\left(C_{3}\right)} .
$$

We obtained $[25,26]$ from the relation (149) the expression for the partial derivative:

$$
\begin{aligned}
\frac{\partial}{\partial t} \psi_{3, \mathrm{MOON}}\left(C_{3}\right) & \\
= & \frac{\gamma M_{\mathrm{MOON}} e_{\mathrm{MOON}} p_{\mathrm{MOON}} \sin \left(\varphi_{\mathrm{MOON}}(t)\right)}{\left(p_{\mathrm{MOON}}+p_{E}\right)^{2}} \\
& \times \frac{d \varphi_{\mathrm{MOON}}(t)}{d t}
\end{aligned}
$$

We can obtain the focal parameter $p_{\text {MOON }}$ of the elliptical orbit of the Moon in terms of the average distance $R_{3 M}$ (between the mass centers of the Earth and the Moon) and the eccentricity $e_{\text {MOON }}=e_{E}$ of the elliptical orbits of the Moon and the Earth around the combined mass center $C_{3, \mathrm{MOON}}$ of the Earth and the Moon. Using the relation (143), we have the relation for the large semiaxis $a_{\text {MOON }}$ of the elliptical orbit of the Moon:

$$
\begin{aligned}
2 a_{\mathrm{MOON}} & =r_{\mathrm{MOON}}(0)+r_{\mathrm{MOON}}(\pi) \\
& =\frac{p_{\mathrm{MOON}}}{\left(1+e_{\mathrm{MOON}}\right)}+\frac{p_{\mathrm{MOON}}}{\left(1-e_{\mathrm{MOON}}\right)} \\
& =\frac{2 p_{\mathrm{MOON}}}{\left(1-e_{\mathrm{MOON}}^{2}\right)},
\end{aligned}
$$

which gives the relation for the focal parameter $p_{\mathrm{MOON}}$ of the elliptical orbit of the Moon:

$$
p_{\mathrm{MOON}}=\left(1-e_{\mathrm{MOON}}^{2}\right) a_{\mathrm{MOON}} .
$$

We can obtain the large semiaxis $a_{\text {MOON }}$ of the elliptical orbit of the Moon in terms of the average distance $R_{3 M}$ (between the mass centers of the Earth and the Moon) and the eccentricity $e_{\mathrm{MOON}}$ of the elliptical orbit of the Moon. Defining the average distance $R_{3 M}$ (between the mass centers of the Earth and the Moon) as the average arithmetic value of the large semiaxis $a_{\mathrm{MOON}}$ and the small semiaxis $b_{\mathrm{MOON}}$ of the elliptical orbit of the Moon

$$
R_{3 M}=\frac{\left(a_{\mathrm{MOON}}+b_{\mathrm{MOON}}\right)}{2}
$$


and using the definition of the eccentricity $e_{\mathrm{MOON}}$ of the elliptical orbit of the Moon

$$
e_{\mathrm{MOON}}=\frac{c_{\mathrm{MOON}}}{a_{\mathrm{MOON}}}=\frac{\sqrt{a_{\mathrm{MOON}}^{2}-b_{\mathrm{MOON}}^{2}}}{a_{\mathrm{MOON}}},
$$

we obtained $[25,26]$ the relation for the average distance $R_{3 M}$ :

$$
R_{3 M}=\frac{a_{\mathrm{MOON}}\left(1+\sqrt{1-e_{\mathrm{MOON}}^{2}}\right)}{2},
$$

which leads to the relation for the large semiaxis $a_{\mathrm{MOON}}$ :

$$
a_{\mathrm{MOON}}=\frac{2 R_{3 M}}{\left(1+\sqrt{1-e_{\mathrm{MOON}}^{2}}\right)} .
$$

Using the relations (152) and (156), we obtained [25, 26] the relation for the focal parameter $p_{\mathrm{MOON}}$ of the elliptical orbit of the of the Moon:

$$
p_{\mathrm{MOON}}=\frac{2 R_{3 M}\left(1-e_{\mathrm{MOON}}^{2}\right)}{\left(1+\sqrt{1-e_{\mathrm{MOON}}^{2}}\right)} .
$$

Using the relation (157) and the relations

$$
\begin{gathered}
p_{\text {MOON }}=p_{\text {MOON }, E} \frac{M_{3}}{\left(M_{3}+M_{\mathrm{MOON}}\right)}, \\
p_{E}=p_{\mathrm{MOON}, E} \frac{M_{\mathrm{MOON}}}{\left(M_{3}+M_{\mathrm{MOON}}\right)},
\end{gathered}
$$

the relation (150) can be rewritten as follows $[25,26]$ :

$$
\begin{aligned}
& \frac{\partial}{\partial t} \psi_{3, \mathrm{MOON}}\left(C_{3}\right) \\
& =\frac{\gamma M_{\mathrm{MOON}} e_{\mathrm{MOON}}\left(1+\sqrt{1-e_{\mathrm{MOON}}^{2}}\right) \sin \left(\varphi_{\mathrm{MOON}}(t)\right)}{2 R_{3 M}\left(1-e_{\mathrm{MOON}}^{2}\right)\left(\frac{M_{\mathrm{MOON}}}{M_{3}}+1\right)^{2}} \\
& \quad \times \frac{d \varphi_{\mathrm{MOON}}(t)}{d t} .
\end{aligned}
$$

Taking $\sin \left(\varphi_{\mathrm{MOON}}(t)\right)=1$ and equating evaluating $d \varphi_{\text {MOON }}(t) / d t$ to $\omega_{\text {MOON }}$ (for the corresponding hypothetical circular orbits of the Earth and the Moon), we obtained [25, 26] the characteristic maximal positive value of the partial derivative $(\partial / \partial t) \psi_{3, \text { MOON }}\left(C_{3}\right)$ :

char. max.pos. $\frac{\partial}{\partial t} \psi_{3, \mathrm{MOON}}\left(C_{3}\right.$, second approximation $)$

$$
=\frac{\gamma M_{\mathrm{MOON}} e_{\mathrm{MOON}}\left(1+\sqrt{1-e_{\mathrm{MOON}}^{2}}\right) \omega_{\mathrm{MOON}}}{2 R_{3 M}\left(1-e_{\mathrm{MOON}}^{2}\right)\left(\left(M_{\mathrm{MOON}} / M_{3}\right)+1\right)^{2}} .
$$

To evaluate (in the second approximation) the relative power of the energy gravitational influence of the Moon on the Earth, we obtained the ratio $f_{\text {MOONM }}\left(C_{3}\right.$, second approximation) of the maximal characteristic maximal positive value char. $\max$.pos. $(\partial / \partial t) \psi_{3, \text { MOON }}\left(C_{3}\right.$, second approximation) and the maximal positive value $\max (\partial / \partial t) \psi_{3 M}\left(C_{3}\right.$, int $)$ :

$$
\begin{aligned}
& f_{\text {MOONM }}\left(C_{3} \text {, second approximation }\right) \\
& =\frac{\text { char. max.pos. }(\partial / \partial t) \psi_{3, \text { MOON }}\left(C_{3} \text {, second approximation }\right)}{\max (\partial / \partial t) \psi_{3 M}\left(C_{3}, \text { int }\right)} .
\end{aligned}
$$

The corresponding numerical value $f_{\text {MOONM }}\left(C_{3}\right.$, second approximation $)=19.44083$ was calculated $[25,26]$ by taking into account the following numerical values: $e_{\mathrm{MOON}}=0.05$, $M_{\mathrm{MOON}}=M_{3} / 81, M_{M}=0.06 M_{3}, T_{\mathrm{MOON}}=29.5306$ days, and $T_{M}=88$ days.

The calculated numerical value $f_{\text {MOONM }}\left(C_{3}\right.$, second approximation) is 19.44083 times larger than the maximal power of the energy gravitational influence (on the unit mass at the mass center $C_{3}$ of the Earth) of Mercury moving around the mass center $O$ of the Sun along the hypothetical circular orbit.

Taking into account the calculated $[25,26]$ nondimensional maximal instantaneous energy gravitational influences on the unit mass of the Earth at the mass center $C_{3}$ of the Earth: $f\left(2, C_{3}\right)=37.69807434$ (for Venus), $f_{\text {MOONM }}\left(C_{3}\right.$, second approximation) $=19.44083404$ (for the Moon), $f(5$, $\left.C_{3}\right)=7.41055774$ (for Jupiter), $f\left(1, C_{3}\right)=1$ (for Mercury), $f\left(4, C_{3}\right)=0.67441034$ (for Mars), $f\left(6, C_{3}\right)=0.24601009$ (for Saturn), $f\left(7, C_{3}\right)=0.00319056$ (for Uranus), $f\left(8, C_{3}\right)=$ 0.00077565 (for Neptune), and $f\left(9, C_{3}\right)=3.4813 \cdot 10^{-8}$ (for Pluto), we have the following order of significance (in the frame of the considered second approximation) of the Moon and the planets of the Solar System: Venus, Moon, Jupiter, Mercury, Mars, Saturn, Uranus, Neptune, and Pluto. The obtained numerical sequence reveals the main instantaneous energy gravitational influences on the Earth of Venus, Moon, Jupiter, Mercury, and Mars, which determine (in col-lection) the main combined instantaneous energy gravitational influence on the Earth (not taking into account the instantaneous energy gravitational influences of the Sun and our Galaxy).

4.2.2. The Evaluation of the Maximal Integral Energy Gravitational Influence of the Moon on the Earth in the Approximation of the Elliptical Orbits of the Earth and the Moon around the Combined Mass Center $C_{3, M O O N}$ of the Earth and the Moon. We evaluated $[25,26]$ the maximal integral energy gravitational influence of the Moon on the Earth in the approximation of the elliptical orbits of the Earth and the Moon around the combined mass center $C_{3, \text { MOON }}$ of 
the Earth and the Moon. We have the integral energy gravitational influence of the Moon on the Earth $\tau_{3}$ (characterized by the mass $\left.M_{3}\right)$ during the time interval $\left(t_{0}, t\right)$ :

$$
\begin{aligned}
\Delta_{g} E_{3} & \left(\text { Moon, } \varphi_{\text {MOON }}\left(t_{0}\right), t, t_{0}\right) \\
& =\int_{t_{0}}^{t}\left(\iiint_{\tau_{3}}\left(\frac{\partial \psi_{3, \mathrm{MOON}}\left(C_{3}\right)}{\partial t^{\prime}}\right) \rho d V\right) d t^{\prime} \\
& \approx M_{3} \int_{t_{0}}^{t}\left(\frac{\partial \psi_{3, \mathrm{MOON}}\left(C_{3}\right)}{\partial t^{\prime}}\right) d t^{\prime}
\end{aligned}
$$

Substituting the expression (159) into (162) and integrating, we obtained $[25,26]$ the following analytical relation:

$$
\begin{aligned}
& \Delta_{g} E_{3}\left(\text { Moon, } \varphi_{\text {MOON }}\left(t_{0}\right), t, t_{0}\right) \\
& =-\left(\gamma M_{3} M_{\mathrm{MOON}} e_{\mathrm{MOON}}\left(1+\sqrt{1-e_{\mathrm{MOON}}^{2}}\right)\right. \\
& \left.\times\left(\cos \varphi_{\mathrm{MOON}}(t)-\cos \varphi_{\mathrm{MOON}}\left(t_{0}\right)\right)\right) \\
& \times\left(2 R_{3 M}\left(1-e_{\mathrm{MOON}}^{2}\right)\left(\frac{M_{\mathrm{MOON}}}{M_{3}}+1\right)^{2}\right)^{-1} .
\end{aligned}
$$

Considering the following phases: $\varphi_{\text {MOON }}(t)=\pi$ and $\varphi_{\text {MOON }}\left(t_{0}\right)=0$, we have from relation (163) the maximal positive value of the integral energy gravitational influence of the Moon on the Earth:

$$
\begin{aligned}
\max _{t} \Delta_{g} E_{3}\left(\text { Moon, } \varphi_{\mathrm{MOON}}\left(t_{0}\right), t, t_{0}\right) \\
=\frac{\gamma M_{3} M_{\mathrm{MOON}} e_{\mathrm{MOON}}\left(1+\sqrt{1-e_{\mathrm{MOON}}^{2}}\right)}{R_{3 M}\left(1-e_{\mathrm{MOON}}^{2}\right)\left(\frac{M_{\mathrm{MOON}}}{M_{3}}+1\right)^{2}} .
\end{aligned}
$$

To evaluate the maximal integral energy gravitational influence of the Moon on the Earth, we consider the ratio $s$ (Moon, second approximation) of the maximal positive value (164) and the maximal positive value (136) (of the integral energy gravitational influence of Mercury on the Earth):

$$
\begin{aligned}
& s \text { (Moon, second approximation) } \\
& =\frac{\max _{t} \Delta_{g} E_{3}\left(\text { Moon, } \varphi_{\mathrm{MOON}}\left(t_{0}\right), t, t_{0}\right)}{\max _{t} \Delta_{g} E_{3}\left(\tau_{1}, 0,0, t, 0\right)} \\
& =\left(e_{\mathrm{MOON}}\left(1+\sqrt{1-e_{\mathrm{MOON}}^{2}}\right) M_{\mathrm{MOON}}\right. \\
& \left.\quad \times\left(R_{\mathrm{O} 3}^{2}-R_{\mathrm{O} 1}^{2}\right)\left(T_{3}-T_{1}\right)\right) \\
& \quad \times\left(\left(1-e_{\mathrm{MOON}}^{2}\right)\left(\frac{M_{\mathrm{MOON}}}{M_{3}}+1\right)^{2} M_{1} R_{3 M R_{\mathrm{O} 1} T_{3}}\right)^{-1}
\end{aligned}
$$

Using the relation (165), we calculated $[25,26]$ the numerical value $s$ (Moon, second approximation) $=13.0693$ for the following numerical values: the eccentricity $e_{\mathrm{MOON}}=$ 0.05 of the elliptical orbits of the Moon and the Earth around the combined mass center $C_{3, \mathrm{MOON}}$, the mass $M_{1}=M_{M}=$ $0.06 M_{3}$ aof Mercury, the mass $M_{\mathrm{MOON}}=M_{3} / 81$ of the Moon, the average distance $R_{3 M}=384400 \mathrm{~km}$ between the mass centers of the Earth and the Moon, the time period $T_{3}=$ 365.3 days of the Earth's circulation around the Sun, the time period $T_{1}=T_{M}=88$ days of Mercury's circulation around the Sun, the average radius $R_{\mathrm{OM}}=R_{\mathrm{O} 1}=57.85 \cdot 10^{6} \mathrm{~km}$ of Mercury's orbit around the Sun, and the average radius $R_{\mathrm{O} 3}=$ $149.6 \cdot 10^{6} \mathrm{~km}$ of the Earth's orbit around the Sun.

Thus, considering the aspect of the cosmic gravitational preparation of the strong earthquakes, we have demonstrated the Venusian $(s(2)=89.6409)$ and Jupiter's $(s(5)=31.319)$ energy gravitational predominance [12] in supplying of the cosmic planetary gravitational energy to the focal region of the preparing earthquakes. We have demonstrated the significant maximal integral energy gravitational influence of the Moon $(s$ (Moon, second approximation $)=13.0693)[25,26]$ on the Earth. Venus, Jupiter, and the Moon induce the main combined (planetary and lunar) integral energy gravitational influence on the Earth. The combined maximal integral energy gravitational influence on the Earth of Mars $(s(4)=$ 2.6396), Saturn $(s(6)=1.036)$, and Mercury $(s(1)=1)$ is one order of the magnitude smaller than the maximal integral energy gravitational influence of Venus. The combined maximal integral energy gravitational influence on the Earth of Uranus $(s(7)=0.0133)$, Neptune $(s(8)=0.003229)$, and Pluto $\left(s(9)=1.4495 \cdot 10^{-7}\right)$ is two orders of the magnitude smaller (i.e., negligible) than the maximal integral energy gravitational influence of Mercury.

\subsection{The Demonstration of the Reality of the Cosmic \\ Energy Gravitational Genesis of Preparation and Triggering of Earthquakes}

4.3.1. The Demonstration of the Real Cosmic Energy Gravitational Genesis of Preparation of Earthquakes. Using the formula (139), we evaluated $[12,25,26]$ the numerical value $E_{g}\left(\tau_{2}, D_{3}, m_{\tau}\right)$ of the integral energy gravitational influence of Venus on the macroscopic continuum region $\tau$ (the focal region of the preparing earthquake) of mass $m_{\tau}$ near the surface point $D_{3}$ of the Earth during the time

$$
T_{g}(2)=t_{1}^{*}(3,2)=\frac{1}{2} \frac{T_{2} T_{3}}{\left(T_{2}-T_{3}\right)}=291.902 \text { days }
$$

of the energy gravitational influence of Venus on the macroscopic continuum region $\tau$ of the Earth. Using the expression (139) for the maximal positive integral energy gravitational influence $E_{g}\left(\tau_{2}, D_{3}, m_{\tau}\right)$ of Venus $(i=2)$ on the macroscopic continuum region $\tau$ of mass $m_{\tau}$ near the surface point $D_{3}$ of 
the Earth, we obtained the obvious estimation for the value $E_{g}\left(\tau_{2}, D_{3}, m_{\tau}\right)$ :

$$
\begin{aligned}
& E_{g}\left(\tau_{2}, D_{3}, m_{\tau}\right) \\
& =2 \gamma m_{\tau} M_{2} \frac{R_{\mathrm{O} 2} T_{3}}{\left(R_{\mathrm{O} 3}^{2}-R_{\mathrm{O} 2}^{2}\right)\left(T_{3}-T_{2}\right)} \\
& =2 \gamma\left(l_{\tau}\right)^{3} \rho_{\tau} M_{2} \frac{R_{\mathrm{O} 2} T_{3}}{\left(R_{\mathrm{O} 3}^{2}-R_{\mathrm{O} 2}^{2}\right)\left(T_{3}-T_{2}\right)}>0,
\end{aligned}
$$

where the final expression for the estimation $E_{g}\left(\tau_{2}, D_{3}, m_{\tau}\right)$ is given for the focal region of the cubical form characterized by the size $l_{\tau}$ of the cube. Considering the following numerical values: $l_{\tau}=10 \mathrm{~km}, \rho_{\tau}=5000 \mathrm{~kg} / \mathrm{m}^{3}$ (the average density of the cubical focal region), and $\gamma=6.67 \cdot 10^{-11} \mathrm{~J} \cdot \mathrm{m} / \mathrm{kg}^{2}$ (the gravitational constant) and using the following known [50] parameters of the Solar System: $T_{V}=T_{2}=224.7$ days, $R_{\mathrm{OV}}=$ $R_{\mathrm{O} 2}=108.1 \cdot 10^{6} \mathrm{~km}$, and $M_{V}=M_{2}=0.82 M_{3}, M_{3}=6$. $10^{24} \mathrm{~kg}, T_{3}=365.3$ days, we calculated $[12,25,26]$ from the expression (167) the numerical value

$$
E_{g}\left(\tau_{2}, D_{3}, m_{\tau}\right)=8.619 \cdot 10^{19} \mathrm{~J}
$$

which is close to the change " $\Delta W \approx 10^{20} \mathrm{~J}$ " [2] of the rotational kinetic energy of the Earth during the strongest earthquakes. The order of magnitude of the estimation (168) for the value $E_{g}\left(\tau_{2}, D_{3}, m_{\tau}\right)$ is consistent with the earlier estimation of the seismotectonic energy $E_{\mathrm{ST}}$ [2], which can discharge in the focal region of the strongest earthquakes:

$$
E_{\mathrm{ST}}\left(\tau_{2}, D_{3}, m_{\tau}\right) \leq E_{g}\left(\tau_{2}, D_{3}, m_{\tau}\right)
$$

It was pointed out [2] that the coincidence of the values $E_{\mathrm{ST}}$ and $\Delta W$ is not the casual fact: it is the indication that the strongest earthquake can be considered as the energy quantum corresponding to the regular change of the rotational regime of the Earth. Using the equivalent generalized differential formulations (50), (56), and (59) of the first law of thermodynamics applied for the focal region $\tau$ of the preparing earthquake, we found strictly the equality of the orders of the magnitude of the values $E_{\mathrm{ST}}$ and $\Delta W$ for the strongest earthquakes. Consequently, the coincidence of the orders of the magnitude of the values $E_{\mathrm{ST}}, \Delta W$, and $E_{g}\left(\tau_{2}, D_{3}, m_{\tau}\right)$ is the indication that the regular changes of the rotational regime of the Earth are related to the regular discharges of the accumulated potential energy (in the different focal regions of earthquakes) supplying of the cosmic gravitational energy influences of the planets of the Solar System, the Sun, and the Moon.

Thus, based on the equivalent generalized differential formulations (50), (56), and (59) of the first law of thermodynamics used for the Earth's macroscopic continuum region $\tau$ (the focal region of the preparing earthquake), we evaluate the reality of the cosmic energy gravitational genesis of the preparation of the earthquakes.
4.3.2. The Demonstration of the Real Cosmic Energy Gravitational Genesis of Triggering of the Preparing Earthquakes. We evaluated [12] the relative (normalized on the maximal integral energy gravitational influence of Mercury $\tau_{1}$ on the Earth) average integral energy gravitational planetary influences corresponding to the time duration $T_{\mathrm{MOON}} / 2$ of the maximal integral energy gravitational influence of the Moon on the Earth. We take into account the time durations of the maximal integral energy gravitational influences on the Earth of the inner planets (Mercury $\tau_{1}$ and Venus $\tau_{2}$ )

$$
T_{g}(i)=t_{1}^{*}(i, 3)=\frac{1}{2} \frac{T_{i} T_{3}}{\left(T_{3}-T_{i}\right)}, \quad(i=1,2),
$$

which are the time durations of supplying of the cosmic planetary gravitational energy from the inner planets $(i=1,2)$ to the focal region of the preparing earthquakes. We take into account the time durations of the maximal integral energy gravitational influences on the Earth of the outer planets (Mars $\tau_{4}$, Jupiter $\tau_{5}$, Saturn $\tau_{6}$, Uranus $\tau_{7}$, Neptune $\tau_{8}$, and Pluto $\left.\tau_{9}\right)$

$$
T_{g}(i)=t_{1}^{*}(3, i)=\frac{1}{2} \frac{T_{i} T_{3}}{\left(T_{i}-T_{3}\right)}, \quad(i=4,5,6,7,8,9)
$$

which are the time durations of supplying of the cosmic planetary gravitational energy from the outer planets $(i=$ $4,5,6,7,8,9)$ to the focal region of the preparing earthquakes.

We defined [12] and calculated the relative values $s(i)$ (normalized on the maximal integral energy gravitational influence of Mercury $(i=1)$ on the Earth) of the maximal integral energy gravitational influences on the Earth of the planets of the Solar System $(i=1,2,4,5,6,7,8,9)$. We evaluated [12] the relative (normalized on the maximal integral energy gravitational influence of Mercury $(i=1)$ on the Earth) average values $e(i)$

$$
e(i)=s(i) \frac{0.5 T_{\mathrm{MOON}}}{T_{g}(i)}, \quad(i=1,2,4,5,6,7,8,9)
$$

of the integral energy gravitational influences on the Earth of the planets of the Solar System corresponding to the time duration $T_{\text {MOON }} / 2$ of the maximal integral energy gravitational influence of the Moon on the Earth.

Using the expression (170) for the time $T_{g}(i)$ of supplying of the cosmic planetary gravitational energy from the inner planets $(i=1,2)$, the expression (171) for the time $T_{g}(i)$ of supplying of the cosmic planetary gravitational energy from the outer planets $(i=4,5,6,7,8,9)$ and the expression (172) for $e(i)$, we calculated the following numerical values [12]: $T_{g}(1)=57.96$ days and $e(1)=0.2547$ (which is one order of magnitude smaller than $s$ (Moon, second approximation $)=13.0693)$ for Mercury; $T_{g}(2)=291.902$ days and $e(2)=4.5342$ (which is smaller than $s$ (Moon, second approximation $)=13.0693$ ) for Venus; $T_{g}(4)=390.0545$ days and $e(4)=0.0999$ (which is significantly smaller than 
$s($ Moon, second approximation $)=13.0693)$ for $\operatorname{Mars;} T_{g}(5)=$ 199.4705 days and $e(5)=2.3182$ (which is smaller significantly than $s$ (Moon, second approximation $)=13.0693$ ) for Jupiter; $T_{g}(6)=189.069$ days and $e(6)=0.0809$ (which is significantly smaller than $s$ (Moon, second approximation) $=13.0693$ ) for Saturn; $T_{g}(7)=184.8506$ days and $e(7)=0.001066$ (which is four orders of the magnitude smaller than $s($ Moon, second approximation $)=13.0693)$ for Uranus; $T_{g}(8)=183.7653$ days and $e(8)=0.0002594$ (which is four-five orders of the magnitude smaller than $s$ (Moon, second approximation) $=13.0693$ ) for Neptune; $T_{g}(9)=$ 183.3905 days and $e(9)=1.1671 \cdot 10^{-8}$ (which is nine orders of the magnitude smaller than $s$ (Moon, second approximation) $=13.0693$ ) for Pluto.

In Section 4.2 we have demonstrated the following order of significance of the planets of the Solar System and the Moon for the cosmic gravitational preparation of the strong earthquakes: Venus $(s(2)=89.6409)$, Jupiter $(s(5)=31.319)$, the Moon ( $s$ (Moon, second approximation) $=13.0693)$, Mars $(s(4)=2.6396)$, Saturn $(s(6)=1.036)$, Mercury $(s(1)=$ $1)$, Uranus $(s(7)=0.0133)$, Neptune $(s(8)=0.003229)$, and Pluto $\left(s(9)=1.4495 \cdot 10^{-7}\right)$. We have the different order of significance of the planets of the Solar System and the Moon related to the defined relative average values $e(i)$ : the Moon $(s$ (Moon, second approximation $)=13.0693)$, Venus $(e(2)=$ 4.5342), Jupiter $(e(5)=2.3182)$, Mercury $(e(1)=0.2547)$, Mars $(e(4)=0.0999)$, Saturn $(e(6)=0.0809)$, Uranus $(e(7)=0.001066)$, Neptune $(e(8)=0.0002594)$, and Pluto $\left(e(9)=1.1671 \cdot 10^{-8}\right)$.

Taking into account the obtained [12] numerical values $e(i)$ for the planets of the Solar System and the numerical value $s$ (Moon, second approximation) $=13.0693$ for the Moon [25, 26], we can recognize the established $[25,26]$ predominant significance of the Moon (along with the minor significance of Venus, Jupiter, and Mercury) as the predominant lunar cosmic trigger mechanism of earthquakes preparing by the combined (planetary and lunar) integral energy gravitational influences on the Earth of Venus, Jupiter, the Moon, Mars, and Mercury.

It was suggested in [51] the hypothesis that the Chandler's wobble of the Earth's pole can be generated by the motion of the rigid kernel of the Earth induced by the disturbances in the Sun-Earth-Moon system. The presented results of Sections 4.1, 4.2, and 4.3 support the stated $[25,26]$ conclusion that the related geophysical phenomena (the small oscillatory motion of the rigid kernel of the Earth relative to the fluid kernel of the Earth [51]; the small oscillation of the Earth's pole (i.e., the Chandler's wobble of the Earth's pole); the small oscillations of the boundary of the Pacific Ocean (i.e., the seismic zone of the Pacific Ring [2]); the oscillations [42], rotations, and deformations $[1,2]$ of the geoblocks weakly coupled with the surrounding plastic layers in all seismic zones of the Earth and the formation of fractures related to the strong earthquakes $[1-5,9-12,23]$ and the planetary cataclysms $[25,26])$ are induced by the combined nonstationary cosmic energy gravitational influence on the Earth of the planets of the Solar System, the Sun, and the Moon.

\subsection{The Cosmic Energy Gravitational Genesis of the Time} Periodicities of the Seismotectonic Activity Induced by the Nonstationary Cosmic Energy Gravitational Influences on the Earth of the Sun, the Moon, Venus, Jupiter, and Mars

\subsubsection{The Time Periodicities of the Maximal (Instantaneous} and Integral) Energy Gravitational Influences on the Earth of the Sun and the Moon, Venus, Jupiter, and Mars

(1) The Time Periodicities of the Maximal (Instantaneous and Integral) Energy Gravitational Influences on the Earth of the Sun-Moon System. If the configuration of the Sun and the Moon is characterized at any time moment by the maximal combined (instantaneous or integral) energy gravitational influence on the Earth, then the Sun and the Moon will have the recurrence of the same configuration after different integer numbers of circulations $\left(j_{\mathrm{MOON}, 3}\right.$ circulations of the Moon around the Earth and $m_{3, \text { MOON }}$ circulations of the Earth around the Sun) to satisfy the following condition [12]:

$$
j_{\mathrm{MOON}, 3} T_{\mathrm{MOON}}=m_{3, \mathrm{MOON}} T_{3} .
$$

Following the known method [52], we present the ratio $T_{3} / T_{\text {MOON }}$ by the following mathematical fraction:

$$
\begin{aligned}
\frac{T_{3}}{T_{\text {MOON }}} & =\frac{365.3}{29.5306}=12+\frac{109328}{295306} \\
& =12+\frac{1}{2+\frac{1}{1+\frac{1}{2+\frac{1}{2+\frac{1}{1+\frac{1}{8+\frac{458}{1204}}}}}}} .
\end{aligned}
$$

Considering the different approximation of the ratio $T_{3} / T_{\text {MooN }}$, we obtained [12] the successive approximations for the time periodicities:

$$
\begin{aligned}
& \left(T_{S-\text { MOON }, 3}\right)_{1}=3 \text { years, } \\
& \left(T_{S-M O O N, 3}\right)_{2}=8 \text { years, } \\
& \left(T_{S-M O O N, 3}\right)_{3}=19 \text { years, } \\
& \left(T_{S-M O O N, 3}\right)_{4}=27 \text { years, } \\
& \left(T_{S-M O O N, 3}\right)_{5}=235 \text { years }
\end{aligned}
$$

of recurrence of the maximal (instantaneous and integral) combined energy gravitational influences of the Sun and the Moon on the Earth in the first, second, third, fourth, and fifth approximations, respectively. We can verify that the time periodicity

$$
\left(T_{S-\mathrm{MOON}, 3}\right)_{3}=\left(T_{S-\mathrm{MOON}, 3}\right)_{1}+\left(T_{S-\mathrm{MOON}, 3}\right)_{2}=11 \text { years }
$$

may be considered approximately as the third approximation of recurrence of the maximal (instantaneous and integral) energy gravitational influences of the Sun-Moon system on the Earth. 
(2) The Time Periodicities of the Maximal (Instantaneous and Integral) Energy Gravitational Influences on the Earth of Venus. If the configuration of the Earth and Venus is characterized at any time moment by the maximal (instantaneous or integral) energy gravitational influence on the Earth, then the Earth and Venus will have the recurrence of the same configuration (in the frame of the real elliptical orbits of the Earth and Venus) after $k_{V, 3}$ circulations of Venus around the Sun and $m_{3, V}$ circulations of the Earth around the Sun to satisfy the following condition [12]:

$$
k_{V, 3} T_{V}=m_{3, V} T_{3}
$$

Following the known method [52], we present the ratio $T_{3} / T_{V}$ by the following mathematical fraction:

$$
\begin{aligned}
\frac{T_{3}}{T_{V}} & =\frac{365.3}{224.7}=1+\frac{1406}{2247} \\
& =1+\frac{1}{1+\frac{1}{1+\frac{1}{1+\frac{1}{2+\frac{1}{280}}}}}
\end{aligned}
$$

Considering the different approximation of the ratio $T_{3} / T_{V}$, we obtained the successive approximations for the time periodicities [12]:

$$
\begin{aligned}
& \left(T_{V, 3}\right)_{1}=3 \text { years, } \\
& \left(T_{V, 3}\right)_{2}=8 \text { years }
\end{aligned}
$$

of recurrence of the maximal (instantaneous and integral) energy gravitational influences of Venus on the Earth in the first and second approximations, respectively. We can verify that the time periodicity

$$
\left(T_{V, 3}\right)_{3}=\left(T_{V, 3}\right)_{1}+\left(T_{V, 3}\right)_{2}=11 \text { years }
$$

may be considered as the third approximation of recurrence of the maximal (instantaneous and integral) energy gravitational influences of Venus on the Earth.

(3) The Time Periodicities of the Maximal (Instantaneous and Integral) Energy Gravitational Influences on the Earth of Jupiter. If the configuration of the Earth and Jupiter is characterized at any time moment by the maximal (instantaneous or integral) energy gravitational influences on the Earth, then the Earth and Jupiter will have the recurrence of the same configuration (in the frame of the real elliptical orbits of the Earth and Jupiter) after $n_{J, 3}$ circulations of Jupiter around the Sun and $m_{3, J}$ circulations of the Earth around the Sun to satisfy the condition [12]

$$
n_{J, 3} T_{J}=m_{3, J} T_{3}
$$

Following the known method [52], we present the ratio $T_{J} / T_{3}$ by the following mathematical fraction:

$$
\frac{T_{J}}{T_{3}}=\frac{4332}{365.3}=11+\frac{1}{1+\frac{1}{6+\frac{1}{12+\frac{24}{41}}}}
$$

Considering the different approximation of the ratio $T_{J} / T_{3}$, we obtained the successive approximations for the time periodicities [12]:

$$
\begin{aligned}
& \left(T_{J, 3}\right)_{1}=11 \text { years, } \\
& \left(T_{J, 3}\right)_{2}=12 \text { years, } \\
& \left(T_{J, 3}\right)_{3}=83 \text { years }
\end{aligned}
$$

of recurrence of the maximal (instantaneous and integral) energy gravitational influences of Jupiter on the Earth in the first, second, and third approximations, respectively.

(4) The Time Periodicities of the Maximal (Instantaneous and Integral) Energy Gravitational Influences on the Earth of Mars. If the configuration of the Earth and Mars is characterized at any time moment by the maximal (instantaneous or integral) energy gravitational influence on the Earth, then the Earth and Mars will have the recurrence of the same configuration after $g_{\text {MARS }, 3}$ circulations of Mars around the Sun and $m_{3, \text { MARS }}$ circulations of the Earth around the Sun to satisfy the following condition [12]:

$$
g_{\text {MARS }, 3} T_{\text {MARS }}=m_{3, \text { MARS }} T_{3} .
$$

Following the known method [52], we present the ratio $T_{\text {MARS }} / T_{3}$ by the mathematical fraction:

$$
\begin{aligned}
\frac{T_{\text {MARS }}}{T_{3}} & =\frac{687.0}{365.3}=1+\frac{3217}{3653} \\
& =1+\frac{1}{1+\frac{1}{7+\frac{1}{2+\frac{1}{1+\frac{1}{1+\frac{47}{59}}}}}}
\end{aligned}
$$

Considering the different approximation of the ratio $T_{\text {MARS }} / T_{3}$, we obtained the successive approximations for the time periodicities [12]:

$$
\begin{aligned}
& \left(T_{\text {MARS }, 3}\right)_{1}=15 \text { years, } \\
& \left(T_{\text {MARS }, 3}\right)_{2}=32 \text { years, } \\
& \left(T_{\text {MARS }, 3}\right)_{3}=47 \text { years }
\end{aligned}
$$

of recurrence of the maximal (instantaneous or integral) energy gravitational influences of Mars on the Earth in the first, second, and third approximations, respectively. 
4.4.2. Empirical Time Periodicities of the Seismotectonic Activity of the Earth. It was pointed out [1] that the sinusoidal "saw-like" form of the graphic dependence of growth and recession of the seismotectonic activization of the separate geological structure is related to the following empirical time periodicities:

$$
\begin{aligned}
& 11 \text { years, } \\
& 22 \text { years, } \\
& 44 \text { years, } \\
& 88 \text { years, } \\
& 352 \text { years. }
\end{aligned}
$$

It was pointed out [2] that the strongest earthquakes in the all boundary region of the Pacific Ocean are characterized by the established tendency for recurrence on average once during the following time period [53-61]:

$$
T_{r}=100 \pm 50=50 \div 150 \text { years. }
$$

It was pointed out [2] also that the close values for recurrence of the strongest earthquakes were established for different seismic belts of the Earth: $90 \div 140$ years for the Caucasus [62] and 150 years for the Anatolian fault zone [63]. We present the data of the monograph [2] concerning to the recurrence of the strongest earthquakes in different regions of the seismic zone of the Pacific Ring $[1,64,65]$ :

$$
\begin{aligned}
90 \pm 40 & =50 \div 130 \text { years-Kamchatka, } \\
130 \pm 50 & =80 \div 180 \text { years-Japan, } \\
110 \pm 50 & =60 \div 160 \text { years-Peru, } \\
100 \pm 50 & =50 \div 150 \text { years-Aleutians. }
\end{aligned}
$$

The empirical range of the time periodicities [66] was revealed:

$$
250 \div 300 \text { years }
$$

for recurrence of the strongest earthquakes in Turkey.

The empirical range of the time periodicities [67] was revealed (as a result of analysis of Chinese earthquakes during the time $\Delta t=2200$ years [2] of long observations):

$$
240 \div 280 \text { years }
$$

for recurrence of the strongest earthquakes in China.

Based on the data presented in the monograph [2] concerning the recurrence of the strongest earthquakes in different regions of the Earth, Dr. A. V. Vikulin made the valid conclusion that the seismic processes have the global nature for the Earth. In the next section we shall explain the considered empirical time periodicities by the different combinations of the cosmic energy gravitational influences on the Earth of the Sun-Moon system, Venus, Jupiter, and Mars.
4.4.3. The Time Periodicities of the Periodic Global Seismotectonic (and Volcanic) Activity and the Global Climate Variability of the Earth Induced by the Different Combinations of the Cosmic Energy Gravitational Influences of the Sun-Moon System, Venus, Jupiter, and Mars. We have shown [12] that the periodic recurrence (characterized by the time periodicity $T_{\text {energy }}$ ) of the maximal integral energy gravitational influences on the Earth (defined by the planetary combination of the Sun-Moon system and the arbitrary combination of the planets: Venus, Mars, and Jupiter) leads (according to the generalized differential formulations (50) applied for the Earth) to the periodic recurrence of the maximal tectonic activity (characterized by the same time periodicity $T_{\text {tec }}=T_{\text {energy }}$ ) of the geospheres of the Earth and each geoblock of Earth. We have shown [12] that the periodic recurrence (characterized by the time periodicity $T_{\text {tec }}=T_{\text {energy }}$ ) of the maximal tectonic (and volcanic) activity of the geospheres of the Earth and each geoblock of the Earth (defined by the combination of the SunMoon system and the arbitrary combinations of the planets: Venus, Mars, and Jupiter) leads to the periodic recurrence (characterized by the time periodicity $T_{\text {tec }}=T_{\text {energy }}$ ) of the maximal concentration of the atmospheric greenhouse gases owing to the periodic increase (characterized by the time periodicity $T_{\text {tec }}=T_{\text {energy }}$ ) of the output of the greenhouse gases related to the periodic tectonic-volcanic activization of the Earth. We have shown [12] that the periodic increase (characterized by the time periodicity $T_{\text {tec }}=T_{\text {energy }}$ ) of the average planetary concentration of the atmospheric greenhouse gases leads (as a consequence of the greenhouse effect produced by the gravity-induced periodic tectonic-volcanic activization accompanied by increase of the atmospheric greenhouse gases) to the periodic global planetary warming related to the increase (characterized by the time periodicity $\left.T_{\text {tec }}=T_{\text {energy }}\right)$ of temperature of the atmosphere-ocean system of the Earth. The periodic decrease (characterized by the time periodicity $T_{\text {tec }}=T_{\text {energy }}$ ) of the average planetary concentration of the atmospheric greenhouse gases leads (as a consequence of the decreased greenhouse effect) to the periodic global planetary cooling [12] related to the fall (characterized by the time periodicity $T_{\text {tec }}=T_{\text {energy }}$ ) of temperature of the atmosphere-ocean system of the Earth. The time periodicity $T_{\text {energy }}$ of the periodic recurrence of the maximal integral energy gravitational influences on the Earth (defined by the combination of the Sun-Moon system and the arbitrary combinations of the planets: Venus, Mars, and Jupiter) corresponds to different two time periodicities of the Earth's climate variability [12]: the first time periodicity $T_{\text {clim } 1}=T_{\text {tec }}=T_{\text {energy }}$ (related to the periodic tectonicvolcanic activity of the geospheres of the Earth induced by the cosmic nonstationary combined energy gravitational influences on the Earth of the Sun-Moon system, Venus, Mars, and Jupiter) and the second time periodicity $T_{\text {clim2 }}=T_{\text {endog }}=$ $T_{\text {energy }} / 2$ related to the periodic tectonic-endogenous heating (of the geospheres of the Earth, the atmosphere, and the oceans of the Earth) induced by the periodic continuum deformation (characterized by the time periodicity $T_{\text {energy }}$ ) owing to the periodic cosmic nonstationary combined energy gravitational influences (characterized by the time periodicity $T_{\text {energy }}$ ) on the Earth of the Sun-Moon system, Venus, Mars, and Jupiter. 
Using the equivalent generalized differential formulations (50), (56), and (59) of the first law of thermodynamics [12] applied for the Earth and the evaluated successive approximations for the time periodicities of the periodic recurrence of the maximal (instantaneous and integral) energy gravitational influences on the Earth of the Sun-Moon system, Venus, Jupiter, and Mars, we founded [12] the sets of the global seismotectonic and volcanic periodicities $T_{\text {tec }}$ (of the periodic global gravity-induced seismotectonic and volcanic activities) and the global climatic periodicities $T_{\text {clim1 }}$ (of the periodic global gravity-induced climate variability and the global variability of the quantities of the fresh water and glacial ice resources):

$$
\begin{aligned}
T_{\text {tec }} & =T_{\text {clim } 1}=T_{\text {energy }} \\
& =\left(T_{S \text {-MOON }, 3}\right)_{i}^{l_{o}} \times\left(T_{V, 3}\right)_{j}^{l_{2}} \times\left(T_{\text {MARS }, 3}\right)_{k}^{l_{4}} \times\left(T_{J, 3}\right)_{n}^{l_{5}},
\end{aligned}
$$

determined by the multiplications of various successive time periodicities related to the different combinations of the following integer numbers: $i=1,2,3,4,5 ; j=1,2 ; k=$ $1,2,3 ; n=1,2,3 ; l_{o}=0,1 ; l_{2}=0,1 ; l_{4}=0,1 ; l_{5}=0,1$. The global seismotectonic and volcanic periodicities $T_{\text {tec }}$ and the global climatic periodicities $T_{\text {climl }}$ are related to the periodic recurrence of the maximal combined integral energy gravitational influences on the Earth induced by the different combinations of the cosmic nonstationary energy gravitational influences of the Sun-Moon system, Venus, Jupiter, and Mars.

By comparing the combined time periodicities (obtained from the expression (201)) with the empirical time periodicities of the seismotectonic activity of the Earth submitted in Section 4.4.2, we established [12] that the empirical time periodicities (of the seismotectonic activity of the Earth) may be satisfactory approximated by the expression (201) with the different combinations of the various integer numbers.

The calculated time periodicity 24 years (given by $3 \times 8$ years determined by the Sun-Moon system and Venus) is close to empirical time periodicity 22 years given by (190). The calculated average time periodicity 34.5 years (of the evaluated time range $33 \div 36=3 \times(11 \div 12)$ years $[12,25,26]$ of the Earth's periodic seismotectonic and volcanic activity and the global climate variability determined by the combined cosmic nonstationary energy gravitational influence on the Earth of the Sun-Moon system, Venus, and Jupiter) is very close to the empirical time periodicity 35 years [68] of the sesmotectonic activity of various regions of the seismic belts around the Pacific Ocean (the Pacific Ring). The calculated time periodicity 45 years (given by $3 \times 15$ years determined by the SunMoon system, Venus, and Mars) is close to the empirical time periodicity 44 years given by (191).

The empirical time periodicity 88 years (given by (192)) is equal to the same time periodicity 88 years (given by $8 \times$ 11 years determined by the Sun-Moon system, Venus, and Jupiter). Since the ratio 88 years $/ T_{\text {MARS }}=46.786$ in close to 47 , we concluded $[25,26]$ that the time periodicity 88 years is determined also by Mars.
The calculated time periodicities $88 \div 96$ years (given by $8 \times(11 \div 12)$ years determined by the Sun-Moon system, Venus, and Jupiter) get into the four empirical ranges of the time periodicities: $T_{r}=100 \pm 50$ years (given by (194)), $90 \pm 40$ years (given by (195)), $130 \pm 50$ years (given by (196)), $110 \pm 50$ years (given by (197)), and $100 \pm 50$ years given by (198). The calculated time periodicity 96 years (given by $3 \times 32$ years determined by the Sun-Moon system, Venus, and Mars) gets into the four empirical ranges of the time periodicities: $T_{r}=$ $100 \pm 50$ years (given by (194)), $90 \pm 40$ years (given by (195)), $130 \pm 50$ years (given by (196)), $110 \pm 50$ years (given by (197)), and $100 \pm 50$ years given by $(198)$. The calculated range $99 \div 108$ years (given by $3 \times 3 \times(11 \div 12)$ years determined by the Sun-Moon system, Venus, and Jupiter) gets into the empirical ranges of the time periodicities: $T_{r}=100 \pm 50$ years given by (194), $90 \pm 40$ years (given by (195)), $130 \pm 50$ years (given by (196)), $110 \pm 50$ years (given by (197)), and $100 \pm 50$ years given by (198). The calculated time periodicity 120 years (given by $8 \times 15$ years determined by the Sun-Moon system, Venus, and Mars) gets into the four empirical ranges of the time periodicities: $T_{r}=100 \pm 50$ years (given by (194)), $90 \pm 40$ years (given by (195)), $130 \pm 50$ years (given by (196)), $110 \pm 50$ years (given by (197)), and $100 \pm 50$ years given by (198). The calculated time periodicity 135 years (given by $3 \times 3 \times 15$ years determined by the Sun-Moon system, Venus, and Mars) gets into the empirical range $T_{r}=100 \pm 50$ years given by (194). The calculated time periodicity 152 years (given by $152=$ $19 \times 8$ determined by the Sun-Moon system and Venus) gets into the empirical range $130 \pm 50$ years (given by (196)) and gets into the empirical range $110 \pm 50$ years given by (197). The calculated range $165 \div 180$ years (given by $15 \times(11 \div 12)$ years determined by the Sun-Moon system, Mars, and Jupiter) gets into the empirical range $130 \pm 50$ years given by (196). The calculated time periodicity 249 years (given by $249=3 \times$ 83 years determined by the Sun-Moon system, Venus, and Jupiter) is near the lower value of the empirical range $250 \div 300$ years (given by (199)) and gets into the empirical range $240 \div$ 280 years given by $(200)$. The calculated time periodicity 285 years (given by $19 \times 15$ years determined by the Sun-Moon system and Mars) is close to the upper value of the empirical range $240 \div 280$ years given by $(200)$. The calculated time periodicity 285 years (given by $19 \times 15$ years determined by the Sun-Moon system and Mars) gets into the empirical range $250 \div 300$ years given by (199). The calculated range of the time periodicities $264 \div 288$ years (given by $3 \times 8 \times(11 \div 12$ ) years determined by the Sun-Moon system, Venus, and Jupiter) gets approximately into the empirical range $240 \div 280$ years given by (200). The calculated time periodicity 352 years (given by $32 \times 11$ years determined by the Sun-Moon system, Mars, and Jupiter) is equal to the empirical time periodicity 352 years given by (193).

4.5. Cosmic Energy Gravitational Genesis of the Chinese 2008 Earthquakes. It was forecasted in advance the single Chinese Haicheng 1975 earthquake using the warning omens. The nearest (to the Haicheng 1975 earthquake) Chinese Luhuo 1973 and Tang Shan 1976 earthquakes were not forecasted using the warning omens. The modern Chinese Bachu 2003, 
TABLE 1: The time periods $T_{1}$ (given in years) of recurrence of the strongest Japanese earthquakes [2] and the obtained [12, 25, 26] corresponding time periodicities (given in years) determined by the following cosmic combinations.

\begin{tabular}{|c|c|c|c|c|}
\hline Region & $\begin{array}{l}\text { Magnitude } M \text { of the } \\
\text { strongest Japanese } \\
\text { earthquakes }\end{array}$ & $\begin{array}{l}\text { Date of the } \\
\text { strongest } \\
\text { Japanese } \\
\text { earthquakes }\end{array}$ & $\begin{array}{c}\text { The time periods } T_{1} \text { (given } \\
\text { in years) of recurrence of } \\
\text { the strongest Japanese } \\
\text { earthquakes }\end{array}$ & $\begin{array}{l}\text { Corresponding time periodicities (given in years) } \\
\text { determined by the following cosmic combination }\end{array}$ \\
\hline \multirow{3}{*}{ Tokyo region } & 7.9 & 1.01.1605 & & \\
\hline & 8.2 & 31.12 .1703 & 98 & $\begin{array}{l}88 \div 96=8 \times(11 \div 12) \text {-Sun-Moon-Venus-Jupiter-Mars } \\
96=3 \times 32 \text {-Sun-Moon-Venus-Mars }\end{array}$ \\
\hline & 8.2 & 1.09 .1923 & 220 & $209 \div 228=19 \times(11 \div 12)$-Sun-Moon-Jupiter \\
\hline \multirow{5}{*}{$\begin{array}{l}\text { Southwest } \\
\text { from Tokyo }\end{array}$} & 8.6 & 20.09.1498 & & \\
\hline & 7.9 & 31.01 .1605 & 107 & $\begin{array}{l}96=3 \times 32 \text {-Sun-Moon-Venus-Mars } \\
120=8 \times 15 \text {-Sun-Moon-Venus-Mars }\end{array}$ \\
\hline & 8.4 & 28.10.1707 & 102 & $\begin{array}{l}96=3 \times 32 \text {-Sun-Moon-Venus-Mars } \\
120=8 \times 15-\text { Sun-Moon-Venus-Mars }\end{array}$ \\
\hline & 8.4 & 23.12.1854 & 147 & $152=19 \times 8$-Sun-Moon-Venus \\
\hline & 8.0 & 7.12 .1944 & 90 & $88 \div 96=8 \times(11 \div 12)$-Sun-Moon-Venus-Jupiter \\
\hline
\end{tabular}

Ruichang 2005, and Yanjin 2006 earthquakes were not forecasted also using the warning omens. Using the results of previous sections and the presented set of the Chinese earthquakes (Luhuo 1973, Haicheng 1975, and Tang Shan 1976; Bachu 2003, Ruichang 2005, and Yanjin 2006), we predicted (Simonenko, 2007) the nearest (to the Yanjin 2006 earthquake) Chinese 2008 earthquakes by considering the following arguments.

The difference equal to 3 years between the times of the Luhuo 1973 and Tang Shan 1976 earthquakes means that the Tang Shan 1976 earthquake may be induced by the integral energy gravitational influence of the Sun-Moon system or Venus (characterized by the same time periodicity 3 years of recurrence of the maximal integral energy gravitational influence on the Earth in the first approximation) or by the combined integral energy gravitational influence on the Earth of the Sun-Moon system and Venus. If the Tang Shan 1976 earthquake was induced by the integral energy gravitational influence of the Sun-Moon system then the Bachu 2003 earthquake can be explained by the recurrence of the integral energy gravitational influence of the Sun-Moon system (characterized by the time periodicity 27 years of recurrence of the maximal integral energy gravitational influence on the Earth in the fourth approximation):

$$
1976+27=2003-\text { Bachu } 2003 \text { earthquake. }
$$

The Tang Shan 1976 earthquake can be induced by the participation of the integral energy gravitational influence of Venus (characterized by the time periodicity 8 years of recurrence of the maximal integral energy gravitational influence on the Earth in the second approximation) since it is easily to explain the Ruichang 2005 earthquake by the recurrence of the maximal integral energy gravitational influence of Venus on the Earth. Considering [12]

$$
1973+i_{V} 8=2005
$$

which should be solved for integer number $i_{V}$, we obtained [12] the integer number $i_{V}=4$ confirming the Venusian genesis of the Tang Shan 1976 and the Ruichang 2005 earthquakes:

$1973+4 \times 8=2005$-Ruichang 2005 earthquake.

Taking into account the Chinese Yanjin 2006 earthquake (explained [12] by the sum $1973+3 \times 11$ ) and considering the Venusian genesis of the Tang Shan 1976 earthquake, we obtained the time of the Chinese 2008 earthquake from the following inequality [12]:

$$
0<1976+j_{V} 8-2006<\left(T_{V, 3}\right)_{1}=3
$$

which should be solved for integer number $j_{V}$. The obtained solution $j_{V}=4$ founded the time of the several (owing to the participation of Mars characterized by the time periodicity 32 years of recurrence of the maximal integral energy gravitational influence on the Earth in the second approximation) distinct Chinese 2008 earthquakes [12]:

$$
1976+4 \times 8=2008 \text {. }
$$

The occurrence of two large earthquakes in 2008 in the areas surrounding Yutian (Xinjiang) and Wenchuan (Sichuan) confirmed the proposed $[12,25,26]$ cosmic energy gravitational genesis of the strong Chinese earthquakes.

4.6. Cosmic Energy Gravitational Genesis of the Strongest Japanese Earthquakes near the Tokyo Region. We have shown $[12,25,26]$ the cosmic energy gravitational genesis of the strongest $(M \geq 7.9)$ Japanese earthquakes near Tokyo region and southwest from Tokyo. To confirm the proposed cosmic energy gravitational genesis of the strongest $(M \geq 7.9)$ Japanese earthquakes, we present in Table 1 the time periods $T_{1}$ (given in years) of recurrence of the strongest Japanese earthquakes [2] and the obtained [12, 25, 26] corresponding time periodicities (given in years) determined by the following planetary combinations. 
Taking into account the time periodicity 83 years (given by (185)) and the year $1927 \mathrm{AD}$ of Jupiter's opposition with the Earth, respectively, and the time periodicity 88 years $=8 \times 11$ years (determined by the Sun-Moon system, Venus, Jupiter, and Mars) and the year $1923 \mathrm{AD}$ of the last strongest Japanese earthquake in the Tokyo region, we predicted $[25,26]$ "the time range

$$
2010 \div 2011 \text { AD }(1927+83 \div 1923+88)
$$

of the next sufficiently strong Japanese earthquake near the Tokyo region".

The powerful 6.6-magnitude (on Richter scale) Japanese earthquake (that occurred on 14 March, 2010) near Tokyo (with the epicenter in the Fukushima Prefecture) gets into the predicted time range $2010 \div 2011 \mathrm{AD}[25,26]$. The powerful 6.8-magnitude (on Richter scale) Japanese earthquake (that occurred on 11 March, 2011) near Tokyo gets also into the predicted time range $2010 \div 2011 \mathrm{AD}[25,26]$. The occurrence of the strong Japanese earthquakes on 14 March, 2010, and on 11 March, 2011, confirmed the previous predictions $[1,25,26]$ and the proposed cosmic energy gravitational genesis $[12,25$, 26] of the strongest Japanese earthquakes.

\section{Summary of Main Results and Conclusion}

We have developed in this article the fundamentals of the thermohydrogravidynamic theory intended for the longterm deterministic predictions of the strong earthquakes. The deterministic thermohydrogravidynamic theory is based on the established generalized formulation $[11,12]$ of the first law of thermodynamics for moving rotating deforming compressible heat-conducting stratified macroscopic continuum region $\tau$ subjected to the nonstationary Newtonian gravitational field.

Using the classical continuum-mechanical theoretical approach [29, 33], we have presented in Section 2.1 the generalized expression [24, 31] for the macroscopic kinetic energy of a small continuum region. The generalized expression $[24,31]$ is the generalization of the classical expression $[28,29]$ in classical nonequilibrium thermodynamics for the macroscopic kinetic energy per unit mass of a small macroscopic continuum region (considered in a stratified threedimensional shear flow) by taking into account the irreversible shear component of the hydrodynamic velocity field related to the rate of strain tensor $e_{i j}$. The macroscopic kinetic energy (of the small macroscopic continuum region) is presented as a sum of the macroscopic translational kinetic energy and three Galilean invariants: the classical macroscopic internal rotational kinetic energy [28, 29], the established macroscopic internal shear kinetic energy [24, 31], and the established macroscopic internal kinetic energy of shear-rotational coupling $[24,31]$ with a small correction. The generalized formula (20) for the macroscopic kinetic energy per unit mass $\varepsilon_{k}$ and its particular form (31) for homogeneous continuum regions of spherical and cubical shapes generalize the classical de Groot and Mazur expression (2) in classical nonequilibrium thermodynamics [28, 29] by taking into account the established $[24,31]$ macroscopic internal shear kinetic energy per unit mass $\varepsilon_{s}$, which expresses the kinetic energy of irreversible dissipative shear motion, and also the established [24, 31] macroscopic internal kinetic energy of shear-rotational coupling per unit mass $\varepsilon_{s, r}^{\text {coup }}$, which expresses the kinetic energy of local coupling between irreversible dissipative shear and reversible rigid-like rotational macroscopic fluid motions. The generalized expression (20) confirms the postulate [35] that the velocity shear $\left(e_{i j} \neq 0\right)$ represents an additional energy source.

Based on the postulates of thermodynamics [27, 28], continuum mechanics $[29,30]$ and hydrodynamics $[33,38]$, we have presented in Section 2.2 the equivalent generalized differential formulations (50), (56), and (59) (given for the Galilean frame of reference) of the first law of thermodynamics $[11,12]$ for nonequilibrium shear-rotational states of the deformed finite one-component individual continuum region (characterized by the symmetric stress tensor $\mathbf{T}$ ) moving in the nonstationary Newtonian gravitational field. The equivalent generalized differential formulations (50), (56), and (59) are valid for moving rotating deforming compressible heat-conducting stratified macroscopic continuum region $\tau$ subjected to the nonstationary Newtonian gravity. The generalized differential formulation (56)

$$
d U_{\tau}+d K_{\tau}+d \pi_{\tau}=\delta Q+\delta A_{\mathrm{np}, \partial \tau}+d G
$$

generalizes the classical $[27,32]$ formulations (39) and (40):

$$
d U=\delta Q-p d V, \quad\left(d \varepsilon \equiv d U \equiv d U_{\tau},-\delta W=-p d V\right)
$$

by taking into account (along with the classical infinitesimal change of heat $\delta Q$ and the classical infinitesimal change of the internal energy $d U_{\tau} \equiv d U$ ) the infinitesimal increment of the macroscopic kinetic energy $d K_{\tau}$, the infinitesimal increment of the gravitational potential energy $d \pi_{\tau}$, the generalized expression $[11,12,24]$ for the infinitesimal work $\delta A_{\mathrm{np}, \partial \tau}$ done on the continuum region $\tau$ by the surroundings of $\tau$, and the additional $[11,12]$ infinitesimal amount $d G$ of energy added (or lost) as the result of the Newtonian nonstationary energy gravitational influence on the continuum region $\tau$ during the infinitesimal time interval $d t$. The presented (in Section 2.3) generalized expression [11, 12] for infinitesimal work $\delta A_{\mathrm{np}, \partial \tau}$ (done on the continuum region $\tau$ by the surroundings of $\tau$ ) generalizes the classical [27] expression $\delta A_{n p, \partial \tau}=-\delta W=$ $-p d V$ by taking into account (for Newtonian continuum) the infinitesimal work $\delta A_{c}$ (given by expression (68)) of the acoustic forces and the infinitesimal work $\delta A_{s}$ (given by expression (69)) of the viscous forces acting during the infinitesimal time interval $d t$ on the boundary surface $\partial \tau$ of the individual continuum region $\tau$.

Based on the equivalent generalized differential formulations (50), (56), and (59) of the first law of thermodynamics and the obtained expression (74) for the gravitational energy power $W_{\mathrm{gr}}(\tau)$, we have presented in Section 2.4 the established $[11,12]$ gravitational energy mechanism of the gravitational energy supply into the continuum region $\tau$ owing to the local time increase of the potential $\psi$ of the gravitational field inside the continuum region $\tau$ subjected to the nonstationary Newtonian gravitational field. The revealed gravitational 
mechanism $[11,12]$ of the gravitational energy supply into the continuum region $\tau$ is consistent with the empirical findings $[1,3-5,9,10]$ related to the anomalous variations of the gravitational field before the strong earthquakes. Based on the equivalent generalized differential formulations (50), (56), and (59) of the first law of thermodynamics, we have presented the evidence $[12,25]$ about the significant increase of the energy flux $\delta F_{\text {vis }, c}$ (given by expression (76)) of the geoacoustic energy from the focal region $\tau$ before the earthquake in a good agreement with the results of the detailed experimental studies [43].

We have presented in Section 3 the evidence $[12,25]$ of the cosmic energy gravitational genesis of earthquakes. Using the evolution equation (73) (deduced from the generalized differential formulation (50) of the first law of thermodynamics) of the total mechanical energy of the macroscopic continuum region $\tau$ (of the compressible viscous Newtonian continuum), we have presented in Section 3.1 the thermodynamic foundation of the classical deformational (shear) model [1] of the earthquake focal region for the quasi-uniform medium of the Earth's crust characterized by practically constant viscosity. Based on the generalized differential formulation (50) of the first law of thermodynamics for the macroscopic continuum region $\tau$, we have presented in Section 3.1 the generalized thermohydrogravidynamic shear-rotational model [11, 12] of the earthquake focal region by taking into account the classical macroscopic rotational kinetic energy [28, 29], the macroscopic nonequilibrium kinetic energies [24, 31], and the external cosmic energy gravitational influences on the focal region of the preparing earthquakes. We have presented in Section 3.2 the evidence $[11,12]$ of the physical adequacy of the rotational model [2] of the earthquake focal region for the seismic zone of the Pacific Ring.

We have presented in Section 4 the fundamentals of the cosmic geophysics $[12,25,26]$. We have presented in Section 4.1 the consideration of the energy gravitational influences on the Earth of the inner planets and the outer planets of the Solar System. We have presented in Section 4.1.1 the relation (96) for the energy gravitational influences (on the Earth) of the inner and the outer planets in the second approximation of the elliptical orbits of the planets of the Solar System. We have presented in Section 4.1.2 the evaluation of the relative maximal planetary instantaneous energy gravitational influences on the Earth in the approximation of the circular orbits of the planets of the Solar System. Considering the maximal positive value $\max (\partial / \partial t) \psi_{31}\left(C_{3}\right.$, int) (of the partial derivative $(\partial / \partial t) \psi_{31}\left(C_{3}\right.$, int $)$ of the gravitational potential $\psi_{31}\left(C_{3}\right.$, int $)$ created by Mercury at the mass center $\mathrm{C}_{3}$ of the Earth) as a scale of the instantaneous energy gravitational influence of the planets of the Solar System on the Earth (in the considered first approximation of the circular orbits of the planets), we have presented in Section 4.1.2 the following numerical sequence of the nondimensional relative maximal powers of the planetary energy gravitational influences (on the unit mass of the Earth at the mass center $C_{3}$ of the Earth): $f\left(2, C_{3}\right)=37.69807434$ (for Venus), $f\left(5, C_{3}\right)=7.41055774$ (for Jupiter), $f\left(1, C_{3}\right)=1$ (for Mercury), $f\left(4, C_{3}\right)=0.67441034$ (for Mars), $f\left(6, C_{3}\right)=$ 0.24601009 (for Saturn), $f\left(7, C_{3}\right)=0.00319056$ (for Uranus), $f\left(8, C_{3}\right)=0.00077565$ (for Neptune), and $f\left(9, C_{3}\right)=$ $3.4813 \cdot 10^{-8}$ (for Pluto). Considering the maximal positive value $\max (\partial / \partial t) \psi_{3 M}\left(D_{3}\right.$, int) (of the partial derivative $(\partial / \partial t) \psi_{3 M}\left(D_{3}\right.$, int $)$ of the gravitational potential $\psi_{3 M}\left(D_{3}\right.$, int $)$ created by Mercury at the surface point $D_{3}$ of the Earth) as a scale of the energy gravitational influence of the planets of the Solar System on the Earth (in the considered first approximation of the circular orbits of the planets), we have presented in Section 4.1.2 the following numerical sequence (corrected slightly the previous numerical values [12]) of the nondimensional relative maximal powers of the planetary energy gravitational influences (on the unit mass of the Earth at the surface point $D_{3}$ of the Earth): $f\left(2, D_{3}\right)=37.70428085$ (for Venus), $f\left(5, D_{3}\right)=7.40926122$ (for Jupiter), $f\left(1, D_{3}\right)=$ 1 (for Mercury), $f\left(4, D_{3}\right)=0.67420160$ (for Mars), $f\left(6, D_{3}\right)=0.24596865$ (for Saturn), $f\left(7, D_{3}\right)=0.00319004$ (for Uranus), $f\left(8, D_{3}\right)=0.00077552$ (for Neptune), and $f\left(9, D_{3}\right)=3.4807 \cdot 10^{-8}$ (for Pluto). We have recognized $[25,26]$ that the revealed small difference of the combined planetary maximal energy gravitational influences at the surface point $D_{3}$ and at the mass center $C_{3}$ of the Earth can explain the following related geophysical phenomena: the small oscillatory motion of the rigid kernel of the Earth relative to the fluid kernel of the Earth; the small oscillation of the Earth's pole (i.e., the Chandler's wobble of the Earth's pole); the small oscillations of the boundary of the Pacific Ocean (i.e., the seismic zone of the Pacific Ring); the oscillations, rotations, and deformations of the geoblocks weakly coupled with the surrounding plastic layers in all seismic zones of the Earth and the formation of fractures related to the strong earthquakes and the planetary cataclysms. We have presented in Section 4.1.3 the evaluation of the relative maximal planetary integral energy gravitational influences on the Earth in the approximation of the circular orbits of the planets of the Solar System. Considering the maximal positive integral energy gravitational influence $\max _{t} \Delta_{g} E_{3}\left(\tau_{1}, 0,0, t, 0\right)$ of Mercury (given by the expression (136)) on the Earth as a scale of the integral energy gravitational influence of the planets of the Solar System on the Earth (in the considered first approximation of the circular orbits of the planets), we have presented in Section 4.1.3 the following order of signification (with respect to the established planetary maximal integral energy gravitational influences on the Earth) of the planets of the Solar System: Venus $(s(2)=89.6409)$, Jupiter $(s(5)=31.319)$, Mars $(s(4)=2.6396)$, Saturn $(s(6)=1.036)$, Mercury $(s(1)=1)$, Uranus $(s(7)=0.0133)$, Neptune $(s(8)=$ $0.003229)$, and Pluto $\left(s(9)=1.4495 \cdot 10^{-7}\right)$, which gives the predominant combined planetary integral energy gravitational influence on the Earth of Venus and Jupiter.

We have presented in Section 4.2 the consideration of the lunar energy gravitational influences on the Earth. We have presented in Section 4.2.1 the evaluation of the relative maximal instantaneous energy gravitational influence of the Moon on the Earth in the second approximation of the elliptical orbits of the Earth and the Moon around the combined mass center $C_{3, \text { MOON }}$ of the Earth and the Moon. Considering the maximal positive value $\max (\partial / \partial t) \psi_{31}\left(C_{3}\right.$, int) (of the partial derivative $(\partial / \partial t) \psi_{31}\left(C_{3}\right.$, int) of the gravitational potential 
$\psi_{31}\left(C_{3}\right.$, int $)$ created by Mercury at the mass center $C_{3}$ of the Earth) as a scale for evaluation of the maximal instantaneous energy gravitational influence of the Moon on the Earth, we have presented the nondimensional numerical value $f_{\text {MOONM }}\left(C_{3}\right.$, second approximation $)=19.44083$, which means that the power of the maximal energy gravitational influence of the Moon (on the unit mass of the Earth at the mass center $C_{3}$ of the Earth) is 19.44083 times larger than the maximal power of the energy gravitational influence (on the unit mass at the mass center $C_{3}$ of the Earth) of Mercury moving around the mass center $O$ of the Sun along the hypothetical circular orbit. Taking into account the calculated $[25,26]$ nondimensional maximal instantaneous energy gravitational influences on the unit mass of the Earth at the mass center $C_{3}$ of the Earth, we established the following order of significance (with respect to the established planetary and lunar maximal instantaneous energy gravitational influences on the Earth) of the Moon and the planets of the Solar System: Venus, the Moon, Jupiter, Mercury, Mars, Saturn, Uranus, Neptune, and Pluto, which reveals the main instantaneous energy gravitational influences on the Earth of Venus, the Moon, Jupiter, Mercury, and Mars determining (in collection) the main combined instantaneous energy gravitational influence on the Earth (not taking into account the instantaneous energy gravitational influences of the Sun and our Galaxy).

We have presented in Section 4.2.2 the evaluation of the maximal integral energy gravitational influence of the Moon on the Earth in the approximation of the elliptical orbits of the Earth and the Moon around the combined mass center $C_{3, \mathrm{MOON}}$. Considering the maximal positive integral energy gravitational influence $\max _{t} \Delta_{g} E_{3}\left(\tau_{1}, 0,0, t, 0\right)$ of Mercury (given by the expression (136)) on the Earth as a scale for evaluation of the maximal integral energy gravitational influence of the Moon on the Earth (in the considered first approximation of the circular orbits of the planets and in the approximation of the elliptical orbits of the Earth and the Moon around the combined mass center $C_{3, \mathrm{MOON}}$ ), we established that the maximal positive integral energy gravitational influence of the Moon on the Earth is $s$ (Moon, second approximation) $=$ 13.0693 times larger than the maximal positive integral energy gravitational influence of Mercury on the Earth. Considering the aspect of the cosmic gravitational preparation of the strong earthquakes, we established the Venusian $(s(2)=$ 89.6409 [12]), Jupiter's $(s(5)=31.319$ [12]), and the Moon's $(s($ Moon, second approximation $)=13.0693[25,26])$ energy gravitational predominance in supplying of the cosmic planetary and lunar gravitational energy to the focal region of the preparing earthquakes. By considering the energy gravitational influence on the Earth's macroscopic continuum region $\tau$ of Venus, we have presented in Section 4.3.1 the evidence (based on the equivalent generalized differential formulations (50), (56), and (59) of the first law of thermodynamics used for the macroscopic continuum region $\tau$ considered as the focal region of the preparing earthquake) of the real cosmic energy gravitational genesis of preparation of earthquakes. Using the expression (167) for the maximal positive integral energy gravitational influence $E_{g}\left(\tau_{2}, D_{3}, m_{\tau}\right)$ of Venus on the macroscopic continuum region $\tau$ of mass $m_{\tau}$ near the surface point $D_{3}$ of the Earth, we have presented in Section 4.3.1 the evidence [12] that the regular changes of the rotational regime of the Earth [2] are related to the regular discharges of the accumulated potential energy (in the focal region of earthquakes) supplying of the cosmic energy gravitational influences of the planets of the Solar System, the Sun, and the Moon. We have presented in Section 4.3.2 the evidence of the cosmic energy gravitational genesis of triggering of the preparing earthquakes. By considering the calculated relative average values $e(i)$ of the integral energy gravitational influences (corresponding to the time duration $T_{\mathrm{MOON}} / 2$ of the maximal integral energy gravitational influence of the Moon on the Earth) on the Earth of the planets of the Solar System and the Moon [12, 25]: $s$ (Moon, second approximation) $=$ 13.0693 (for the Moon), $e(2)=4.5342$ (for Venus), $e(5)=$ 2.3182 (for Jupiter), $e(1)=0.2547$ (for Mercury), $e(4)=$ 0.0999 (for Mars), $e(6)=0.0809$ (for Saturn), $e(7)=0.001066$ (for Uranus), $e(8)=0.0002594$ (for Neptune), and $e(9)=$ $1.1671 \cdot 10^{-8}$ (for Pluto), we established [25] the exceptional predominant significance of the cosmic energy gravitational influence of the Moon (along with the established [12] minor significance of Venus, Jupiter, and Mercury) as the predominant cosmic trigger mechanism of discharge (on the final stage of the preparation of the earthquakes) of the accumulated potential energy (in the focal region of earthquakes) supplying of the cosmic gravitational energy influences of the planets of the Solar System and the Moon. The considered results of Sections 4.1, 4.2, and 4.3 have demonstrated the cosmic energy gravitational genesis (related to the combined nonstationary cosmic energy gravitational influence of the planets of the Solar System, the Sun, and the Moon) of the following correlated geophysical phenomena: the small oscillations [2] of the boundary of the Pacific Ocean (i.e., the seismic zone of the Pacific Ring); the small oscillatory motion of the rigid kernel of the Earth relative to the fluid kernel of the Earth [51]; the Chandler's wobble of the Earth's pole; the oscillations [42], rotations, and deformations $[1,2]$ of the geoblocks weakly coupled with the surrounding plastic layers in all seismic zones of the Earth; the formation of the planetary fractures related to the strong earthquakes $[1,3-5,9,10$, $23]$ and the planetary cataclysms $[25,26]$.

We have demonstrated in Section 4.4 the cosmic energy gravitational genesis [12] of the time periodicities of the global seismotectonic activity of the Earth induced by the nonstationary cosmic energy gravitational influences on the Earth of the Sun-Moon system, Venus, Jupiter, and Mars. We have presented in Section 4.4.1 the time periodicities [12] of the maximal (instantaneous and integral) energy gravitational influences on the Earth of the Sun and the Moon, Venus, Jupiter, and Mars. We have presented in Section 4.4.1 (1) the successive approximations of the following time periodicities: $\left(T_{S \text {-MOON }, 3}\right)_{1}=3$ years, $\left(T_{S-\text { MOON }, 3}\right)_{2}=8$ years, $\left(T_{S-M O O N, 3}\right)_{3}=11$ years and $\left(T_{S-M O O N, 3}\right)_{3}=19$ years, $\left(T_{S-M O O N, 3}\right)_{4}=27$ years, and $\left(T_{S-M O O N, 3}\right)_{5}=235$ years of recurrence of the maximal (instantaneous and integral) combined energy gravitational influences of the Sun-Moon system on the Earth in the first, second, third, fourth, and fifth approximations, respectively. We have presented in Section 4.4.1 (2) the successive approximations of the following time periodicities: $\left(T_{V, 3}\right)_{1}=3$ years, $\left(T_{V, 3}\right)_{2}=8$ years, and 
$\left(T_{V, 3}\right)_{3}=11$ years of recurrence of the maximal (instantaneous and integral) energy gravitational influences of Venus on the Earth in the first, second, and third approximations, respectively. We have presented in Section 4.4.1 (3) the successive approximations of the following time periodicities: $\left(T_{J, 3}\right)_{1}=11$ years, $\left(T_{J, 3}\right)_{2}=12$ years, and $\left(T_{J, 3}\right)_{3}=83$ years of recurrence of the maximal (instantaneous and integral) energy gravitational influences of Jupiter on the Earth in the first, second, and third approximations, respectively. We have presented in Section 4.4.1 (4) the successive approximations of the following time periodicities: $\left(T_{\mathrm{MARS}, 3}\right)_{1}=15$ years, $\left(T_{\mathrm{MARS}, 3}\right)_{2}=32$ years, and $\left(T_{\mathrm{MARS}, 3}\right)_{3}=47$ years of recurrence of the maximal (instantaneous or integral) energy gravitational influences of Mars on the Earth in the first, second, and third approximations, respectively. We have presented in Section 4.4.2 the empirical time periodicities [1, 2,53-67] of the seismotectonic activity for different regions of the Earth.

Based on the equivalent generalized differential formulations (50), (56), and (59) of the first law of thermodynamics $[12,25]$ used for the Earth and using the evaluated (in Section 4.4.1) successive approximations for the time periodicities of the periodic recurrence of the maximal energy gravitational influences on the Earth of the Sun-Moon system, Venus, Mars, and Jupiter, we have presented in Section 4.4.3 the following sets of the global seismotectonic and volcanic periodicities $T_{\text {tec }}$ and the global climatic periodicities $T_{\text {clim1 }}$ of the Earth:

$$
\begin{aligned}
T_{\text {tec }} & =T_{\text {clim } 1}=T_{\text {energy }} \\
& =\left(T_{S \text {-MOON }, 3}\right)_{i}^{l_{o}} \times\left(T_{V, 3}\right)_{j}^{l_{2}} \times\left(T_{\text {MARS }, 3}\right)_{k}^{l_{4}} \times\left(T_{J, 3}\right)_{n}^{l_{5}},
\end{aligned}
$$

determined by the successive global periodicities $T_{\text {energy }}$ (defined by the multiplications of various successive time periodicities related to the different combinations of the following integer numbers: $i=1,2,3,4,5 ; j=1,2 ; k=1,2,3$; $\left.n=1,2,3 ; l_{o}=0,1 ; l_{2}=0,1 ; l_{4}=0,1 ; l_{5}=0,1\right)$ of recurrence of the maximal combined energy gravitational influences on the Earth of the different combined combinations of the cosmic nonstationary energy gravitational influences on the Earth of the Sun-Moon system, Venus, Mars, and Jupiter. We have shown that the considered empirical time periodicities $[1,2,53-67]$ of the seismotectonic activity may be satisfactory approximated by the different combined combinations of the cosmic nonstationary energy gravitational influences on the Earth of the Sun-Moon system, Venus, Mars, and Jupiter.

We have presented in Section 4.5 the evidence of the cosmic energy gravitational genesis of the Chinese 2008 earthquakes. Using the established time periodicities [12] of the maximal (instantaneous and integral) energy gravitational influences on the Earth of the Sun-Moon system, Venus, Mars, and Jupiter, and considering the presented set of the Chinese earthquakes (Luhuo 1973, Haicheng 1975, and Tang Shan 1976; Bachu 2003, Ruichang 2005, and Yanjin 2006), we predicted [12] the nearest year (2008) of the next Chinese 2008 earthquakes. The proposed cosmic energy gravitational genesis of the predicted [12] strong Chinese 2008 earthquakes was confirmed by the occurrence of two large earthquakes in 2008 in the areas surrounding Yutian (Xinjiang) and Wenchuan (Sichuan). The proposed cosmic energy gravitational genesis of the predicted [12] strong Chinese 2008 earthquakes was also confirmed by the noticeable gravity variations [9] (derived from regional gravity monitoring data in China from 1998 to 2005) before the occurrence of two large earthquakes in 2008 in the areas surrounding Yutian (Xinjiang) and Wenchuan (Sichuan). According to the equivalent generalized differential formulations (50), (56), and (59) of the first law of thermodynamics $[12,25]$ and the presented results of Section 2.4, the noticeable gravity variations (mentioned in articles $[1,3-5,9,13])$ are related to supply of the gravitational energy into the focal region before the occurrence of earthquake. According to the equivalent generalized differential formulations (50), (56), and (59) of the first law of thermodynamics $[12,25]$, the noticeable gravity variations in the focal region is the necessary (but not sufficient) condition for preparation of earthquake. A recent research [10] by Zhan and his colleagues confirmed this conclusion demonstrating that significant gravity changes were observed before all nine large earthquakes in China from 2001 to 2008. It is clear that the presented equivalent generalized differential formulations (50), (56), and (59) of the first law of thermodynamics $[12,25]$ can give the mathematical basis to "remove the subjective nature in the determination of the timeframe of a forecasted earthquake" [9]. This conclusion is in agreement with the demonstrated (in Section 4.6) cosmic energy gravitational genesis of the strongest Japanese earthquakes near the Tokyo region. "The time range $2010 \div 2011 \mathrm{AD}$ of the next sufficiently strong Japanese earthquake near the Tokyo region" $[25,26]$ was predicted based on the established time periodicities $\left(T_{J, 3}\right)_{3}=83$ years (determined by Jupiter) and 88 years $=8 \times 11$ years (determined by the Sun-Moon system, Venus, Jupiter, and Mars) and taking into account the year $1923 \mathrm{AD}$ of the strongest Japanese earthquake near the Tokyo region. The proposed $[12,25,26]$ cosmic energy gravitational genesis of the strongest Japanese earthquakes was confirmed by occurrence of the strong Japanese earthquakes on 14 March, 2010, and on 11 March, 2011.

The time periodicity 88 years (of the global seismotectonic and volcanic activity and the global climate variability related to recurrence of the maximal combined energy gravitational influences on the Earth of the Sun-Moon system, Venus, Mars, and Jupiter [12, 25, 26]) is in good agreement with the estimated (based on the spectral Fourier analysis) climatic time periodicity 88 years [69] obtained from the studies of sediments from Siberian and Mongolian lakes. This good agreement (of the independent experimental seismotectonic [1] and the climatic [69] periodicity 88 years with the global seismotectonic, volcanic, and climatic periodicity 88 years $[12,25,26])$ is the additional confirmation of the validity of the thermohydrogravidynamic theory $[11,12,25,26]$ of the seismotectonic, volcanic, and climatic activity of the Earth.

The founded generalized differential formulations (50), (56), and (59) of the first law of thermodynamics [12, 25] can be used as the thermohydrogravidynamic basis for rapid development of the urgent technologies of the long-term deterministic predictions of the strong earthquakes in order 
to sustain the stable evolutionary development, the survival, greatness, and cosmic dignity of the humankind in the present and forthcoming epochs of the critical surrounding cosmic, seismotectonic, volcanic, and climatic conditions of the human existence on the Earth.

\section{Acknowledgments}

The author thanks the Academic Editor Dr. Umberta Tinivella with gratitude for the editorial corrections to improve the final text of the article. The author thanks a reviewer for bringing to the author's attention the additional interesting information, which was used with gratitude for the correction of the article. The author thanks Prof. V. A. Abramov and Dr. Sergey V. Smirnov for helpful discussions and Dr. Yuri Panarin and Amrtatjuti V. Sereda for the editorship of the first version of the article in English.

\section{References}

[1] V. A. Abramov, "Forecasting of disastrous earthquakes," Proceedings of the Vladivostok Professor's Club, no. 1, pp. 64-77, 1997 (Russian).

[2] A. V. Vikulin, Physics of Wave Seismic Process, The Kamchatsky State Pedagogical University Press, Petropavlovsk-Kamchatsky, Russia, 2003, In Russian.

[3] B. F. Chao and R. S. Gross, "Changes in the Earth's rotation and low-degree gravitational field induced by earthquakes," Geophysical Journal of the Royal Astronomical Society, vol. 91, no. 3, pp. 569-596, 1987.

[4] B. F. Chao and R. S. Gross, "Changes in the Earth's rotational energy induced by earthquakes," Geophysical Journal International, vol. 122, no. 3, pp. 776-783, 1995.

[5] B. Fong Chao, R. S. Gross, and D.-N. Dong, "Changes in global gravitational energy induced by earthquakes," Geophysical Journal International, vol. 122, no. 3, pp. 784-789, 1995.

[6] F. Machado, "Geological evidence for a pulsating gravitation," Nature, vol. 214, no. 5095, pp. 1317-1318, 1967.

[7] R. H. Rapp, "Gravitational potential of the Earth determined from a combination of satellite, observed, and model anomalies," Journal of Geophysical Research, vol. 73, no. 20, pp. 65556562, 1968.

[8] V. Sgrigna and L. Conti, "A deterministic approach to earthquake prediction," International Journal of Geophysics, vol. 2012, Article ID 406278, 20 pages, 2012.

[9] Y. Zhu and F. B. Zhan, "Medium-term earthquake forecast using gravity monitoring data: evidence from the Yutian and Wenchuan earthquakes in China," International Journal of Geophysics, vol. 2012, Article ID 307517, 6 pages, 2012.

[10] F. B. Zhan, Y. Zhu, J. Ning, J. Zhou, W. Liang, and Y. Xu, "Gravity changes before large earthquakes in China: 1998-2005," GeoSpatial Information Science, vol. 14, no. 1, pp. 1-9, 2011.

[11] S. V. Simonenko, "Statistical thermohydrodynamics of irreversible strike-slip-rotational processes," in Rotational Processes in Geology and Physics, pp. 225-251, KomKniga, Moscow, Russia, 2007, In Russian.

[12] S. V. Simonenko, Thermohydrogravidynamics of the Solar System, Institute of Technology and Business Press, Nakhodka, Russia, 2007.
[13] S. I. Zubkov, Catalogue of Earthquakes' Precursors. Gravitational Precursors, Moscow, Russia, 1988.

[14] T. Matuzawa, "On the possibility of gravitational waves in soil and allied problems," Japanese Journal of Astronomy and Geophysics, vol. 3, pp. 161-177, 1925.

[15] C. Lomnitz and H. Castanos, "Earthquake hazard in the valley of Mexico: entropy, structure, complexity," in Earthquake Source Asymmetry, Structural Media and Rotation Effects, pp. 347-364, Springer, New York, NY, USA, 2006.

[16] C. Lomnitz, "Some observations of gravity waves in the 1960 Chile earthquake," Bulletin of the Seismological Society of America, vol. 59, no. 2, pp. 669-670, 1970.

[17] C. Lomnitz, "Mexico 1985: the case for gravity waves," Geophysical Journal International, vol. 102, no. 3, pp. 569-572, 1990.

[18] C. F. Richter, Elementary Seismology, W.H. Freeman, San Francisco, Calif, USA, 1958.

[19] A. V. Vikulin, "Geodynamics and gravitation (cosmic factors)," in Proceedings of the 3rd Tectonophysic Conference on Thectonophysics and Pressing Questions of the Earth's Sciences, vol. 1, pp. 57-61, UIPE, RAS, Moscow, Russia, 2012, In Russian.

[20] A. V. Vikulin, "Geodynamics and gravitation," in Proceedings of the All-Russian Conference and of Youth School on Modern Geodynamics: Modern Geodynamics of Central Asia and Dangerous Natural Processes: The Results of Investigation Obtained on the Quantitative Basis, pp. 26-28, IEC, SB RAS, Irkutsk, Russia, 2012, In Russian.

[21] A. V. Vikulin, Seismicity, Volcanism and Geodynamics. The Selected Works, The Kamchatsky State University Press, Petropavlovsk-Kamchatsky, Russia, 2011, In Russian.

[22] A. V. Vikulin, “Angular momentum's geodynamics, gravitational waves and the superfluidity of the geological environment," in Proceedings on Seismicity, Volcanism and Geodynamics, pp. 384-394, The Kamchatsky State University Press, Petropavlovsk-Kamchatsky, Russia, 2011, In Russian.

[23] R. Console, K. Yamaoka, and J. Zhuang, "Implementation of short- and medium-term earthquake forecasts," International Journal of Geophysics, vol. 2012, Article ID 217923, 2 pages, 2012.

[24] S. V. Simonenko, Non-Equilibrium Statistical Thermohydrodynamics of Turbulence, Nauka, Moscow, Russia, 2006.

[25] S. V. Simonenko, Fundamentals of the Thermohydrogravidynamic Theory of Cosmic Genesis of the Planetary Cataclysms, Institute of Technology and Business Press, Nakhodka, Russia, 2009.

[26] S. V. Simonenko, Fundamentals of the Thermohydrogravidynamic Theory of Cosmic Genesis of the Planetary Cataclysms, Institute of Technology and Business Press, Nakhodka, Russia, 2nd edition, 2010.

[27] J. W. Gibbs, "Graphical methods in the thermodynamics of fluids," in Transactions of the Connecticut Academy, vol. 2, pp. 309-342, 1873.

[28] S. R. De Groot and P. Mazur, Non-Equilibrium Thermodynamics, North-Holland, Amsterdam, The Netherlands, 1962.

[29] I. Gyarmati, Non-Equilibrium Thermodynamics. Field Theory and Variational Principles, Springer, 1970.

[30] A. Sommerfeld, Vorlesungen Über Theoretische Physic. Bd. $2 I$. Mechanik der Deformierbaren Medien, 2. Neubearb, Aufl, Leipzig, Germany, 1949.

[31] S. V. Simonenko, "The macroscopic non-equilibrium kinetic energies of a small fluid particle," Journal of Non-Equilibrium Thermodynamics, vol. 29, no. 2, pp. 107-123, 2004. 
[32] L. D. Landau and E. M. Lifshitz, Theoretical Physics, vol. 5 of Statistical Physics, Nauka, Moscow, Russia, 1976, In Russian.

[33] G. K. Batchelor, An Introduction to Fluid Dynamics, Cambridge University Press, Cambridge, UK, 1967.

[34] H. Helmholtz, "About integrals of hydrodynamic equations related with vortical motions," Crelle's Journal, vol. 55, p. 25, 1858.

[35] D. J. Evans, H. J. M. Hanley, and S. Hess, "Non-newtonian phenomena in simple fluids," Physics Today, vol. 37, no. 1, pp. 26-33, 1984.

[36] I. Prigogine and I. Stengers, Order Out of Chaos: Man's New Dialogue with Nature, Bantam Books, Toronto, Canada, 1984.

[37] G. Nicolis and I. Prigogine, Exploring Complexity. An Introduction, Freeman, New York, NY, USA, 1989.

[38] L. D. Landau and E. M. Lifshitz, Theoretical Physics, vol. 6 of Hydrodynamics, Nauka, Moscow, Russia, 1988, In Russian.

[39] J. Verhoogen, F. J. Turner, L. E. Weiss et al., The Earth. An Introduction to Physical Geology, Holt, Rinehart and Winston, New York, NY, USA, 1970.

[40] B. Ranguelov, S. Dimitrova, D. Gospodinov et al., "Fractal properties of the mediterranean seismotectonic model for seismic hazard assessment," Annual, Geology and Geophysics, vol. 46, part 1, pp. 397-401, 2003.

[41] S. V. Simonenko, Non-Equilibrium Statistical Thermohydrodynamics, vol. 2 of Towards the Foundation of the Theory of the Non-Equilibrium Dissipative Small-Scale Turbulence and the Tolerance Theory Related with the Quality Control, The Pacific State University of Economics Press, Vladivostok, Russia, 2005.

[42] G. I. Dolgikh, The Investigations of the Wave Fields of the Ocean and Lithosphere By Laser-Interference Methods, Dalnauka, Vladivostok, Russia, 2000, In Russian.

[43] G. I. Dolgikh, A. V. Kuptsov, I. A. Larionov et al., "Deformation and acoustic precursors of earthquakes," Doklady Earth Sciences, vol. 413, no. 1, pp. 281-285, 2007 (Russian).

[44] L. I. Sedov, Mechanics of Continuous Medium, vol. 2, Nauka, Moscow, Russia, 1994, In Russian.

[45] S. Shamsi and F. D. Stacey, "Dislocation models and seismomagnetic calculation for California 1906 and Alaska 1964 earthquakes," Bulletin of the Seismological Society of America, vol. 59, no. 4, pp. 1435-1448, 1969.

[46] V. S. Mount and J. Suppe, "State of stress near the San Andreas fault: implications for wrench tectonics," Geology, vol. 15, no. 12, pp. 1143-1146, 1987.

[47] Z. Guo, "Significance of a ground and block rotation in the earthquake prediction," Northwestern Seismological Journal, vol. 10, no. 1, pp. 82-85, 1988.

[48] A. V. Vikulin and I. V. Melekestcev, "Vortexes and life," in Rotational Processes in Geology and Physics, pp. 39-101, KomKniga, Moscow, Russia, 2007, In Russian.

[49] T. Yu. Tveritinova and A. V. Vikulin, "Wave rotational-elastic tectonics of the planets," in Rotational Processes in Geology and Physics, pp. 271-278, KomKniga, Moscow, Russia, 2007, In Russian.

[50] A. V. Zhirmunsky and V. I. Kuzmin, Critical Levels in the Development of Natural Systems, Nauka, Leningrad, Russia, 1990, In Russian.

[51] Y. N. Avsjuk, Tidal Forces and Natural Processes, UIPE RAS, Moscow, Russia, 1996, In Russian.

[52] Y. I. Perelman, Entertaining Astronomy, State Publishing Office of the Technical-Theoretical Literature, Moscow, Russia, 1956, In Russian.
[53] S. A. Fedotov, "Regularities of the distribution of strong earthquakes in Kamchatka, the Kuril Islands, and Northeastern Japan," Trudy Institut Fiziki Zemli. Akademii Nauk SSSR, vol. 36, no. 203, pp. 63-93, 1965 (Russian).

[54] C. Davison, Great Earthquakes, Thomas Murby, London, UK, 1936.

[55] D. H. Christensen and L. J. Ruff, "Rupture process of the March 3, 1985 Chilean earthquake," Geophysical Research Letters, vol. 13, no. 8, pp. 721-724, 1986.

[56] S. E. Barrientos and E. Kausel, "Genesis y proceso de rupture del terremoto del 3 demarzo de 1985," Revista de Geofísica, vol. 46, no. 1, pp. 3-18, 1990.

[57] K. H. Jacob, "Estimates of long-term probabilities for future great earthquakes in the Aleutians," Geophysical Research Letters, vol. 11, no. 4, pp. 295-298, 1984.

[58] K. Shimazaki and T. Nakata, "Time-predictable recurrence model for large earthquakes," Geophysical Research Letters, vol. 7, no. 4, pp. 279-282, 1980.

[59] S. Suyehiro, "Earthquake prediction efforts in Japan," in Atti della Conferenza Internazionale sulle Zone Sismiche dell'Area Mediterranea, Matera, Italy, 16-18 November 1981, pp. 41-44, Potenza, Italy, 1984.

[60] R. H. Clark, R. R. Dibble, H. E. Fyfe, G. J. Lensen, and R. P. Suggarte, "Tectonic and earthquake risk zoning," Transactions of the Royal Society of New Zealand, vol. 1, no. 10, pp. 113-126, 1965.

[61] A. R. Johnston, "Earthquake fault Line hazards with special reference to Wellington," New Zealand Engineering, vol. 20, no. 8, pp. 320-322, 1965.

[62] G. P. Tamrazyan, "About periodicity of seismic activity during the last one and half-two thousand years (as an example for Armenia)," lzvestiya Akademii Nauk SSSR, Fizika Zemli, no. 1, pp. 76-85, 1962 (Russian).

[63] N. N. Ambraseys, "Some characteristic features of the Anatolian fault zone," Tectonophysics, vol. 9, no. 2-3, pp. 143-165, 1970.

[64] A. V. Vikulin, "Seismicity and the Earth's rotation," Computing Technologies, vol. 1, no. 3, pp. 124-130, 1992 (Russian).

[65] A. V. Vikulin, "On the nature of Australian earthquakes," Volcanology and Seismology, no. 2, pp. 99-108, 1994 (Russian).

[66] I. V. Kyrillov, "On the periodicity of destructive earthquakes at the Caucasus and in Turkey," Doklady Akademii Nauk SSSR, vol. 115, no. 4, pp. 771-773, 1957 (Russian).

[67] H. H. Turner, "On Mr Leath's intersects," Monthly Notices of the Royol Astronomical Society, vol. 46, supplement 1, no. 6, 1925.

[68] S. Hattory, "Migration and periodicity of seismic activity in the world," Bulletin of the International Institute of Seismology, vol. 15, pp. 33-47, 1977.

[69] I. Kalugin and A. Darin, "High resolution geochemical signal of paleoclimate in the bottom sediments based on scanning $\mathrm{X}$-ray fluorescence analysis on synchrotron radiation (XRF SR)," in Abstracts of the 2nd Russia-China Symposium on Marine Science: Marine Environmental and Resources in 21st Century, p. 70, FEB RAS, Vladivostok, Russia, October 2012. 

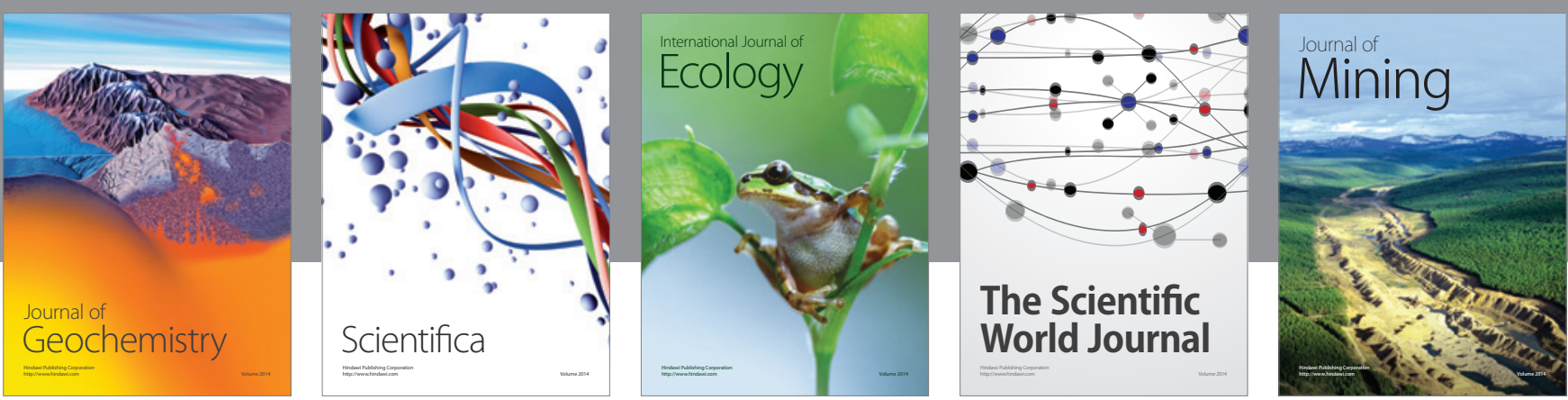

The Scientific World Journal


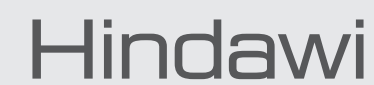

Submit your manuscripts at

http://www.hindawi.com
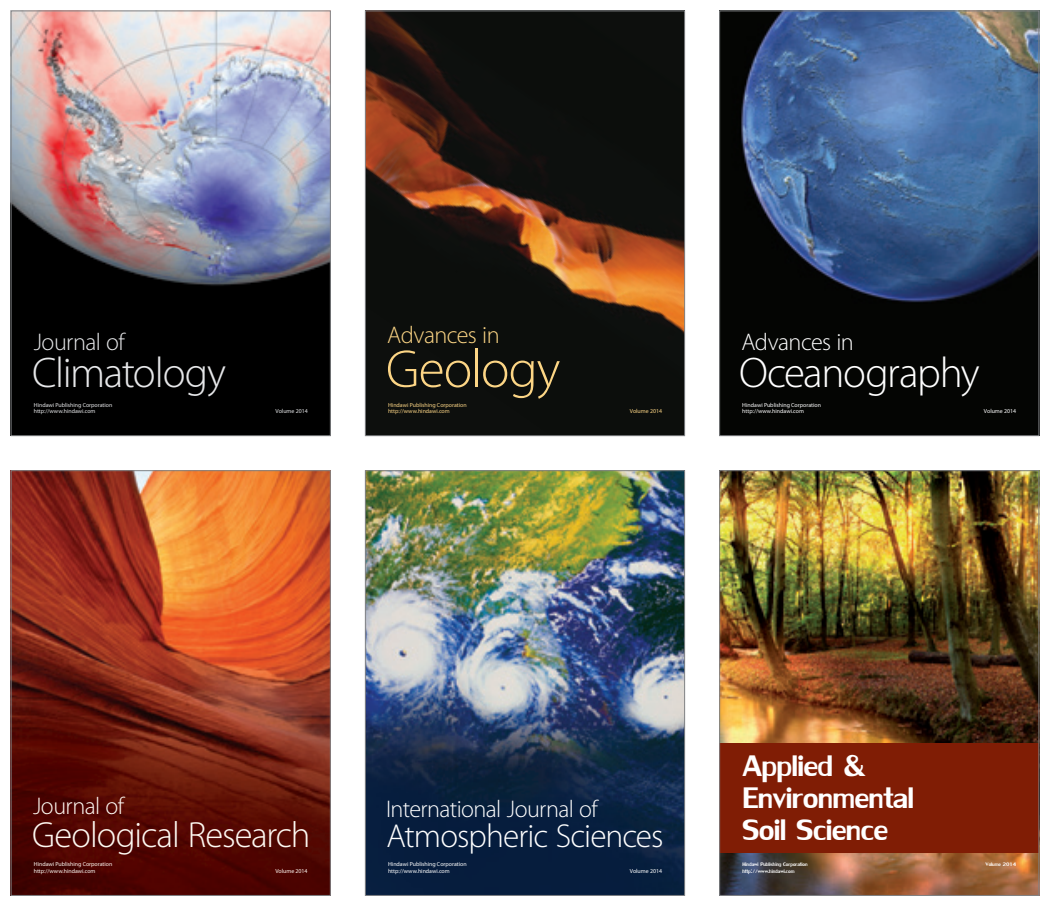
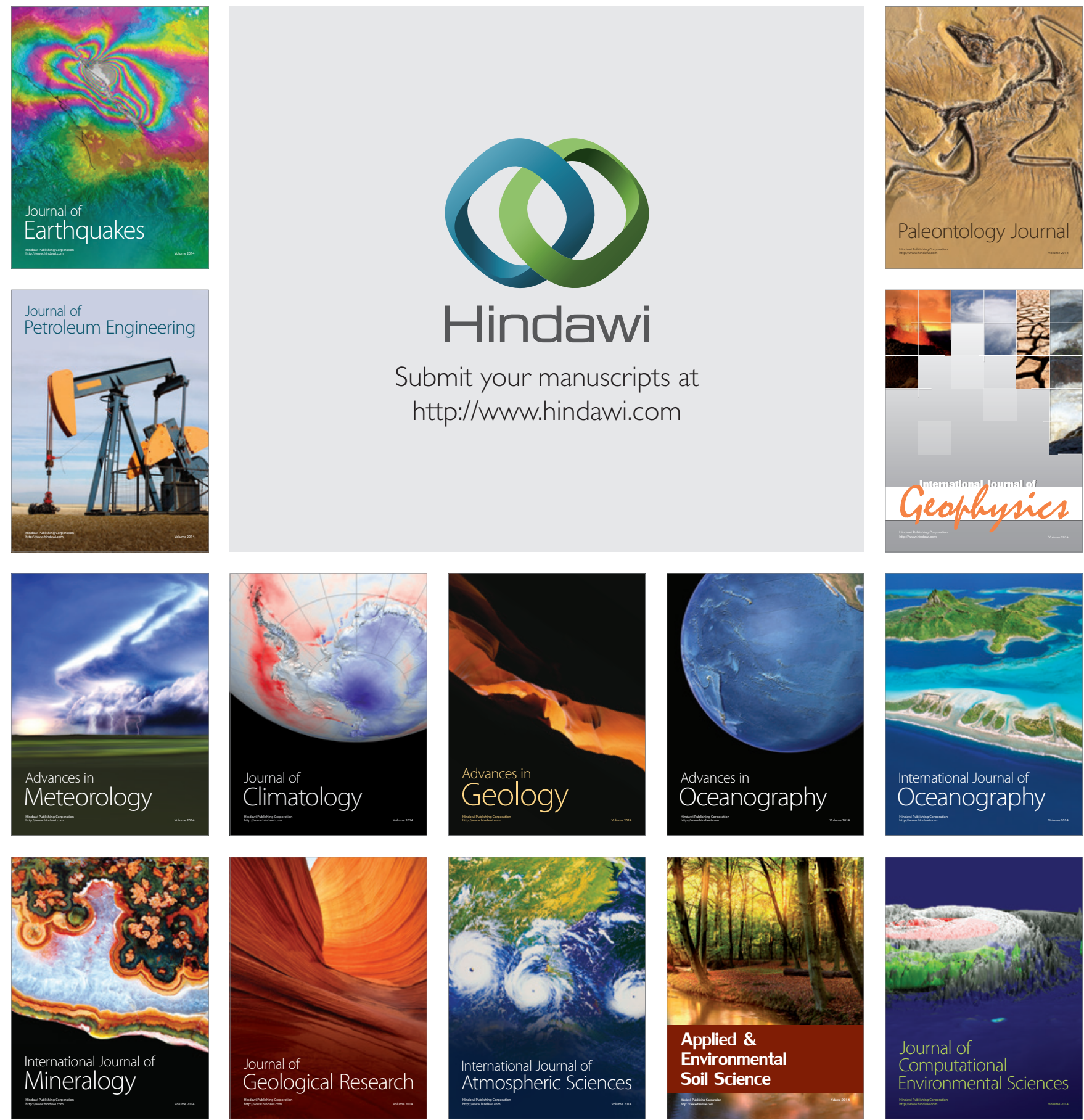